\title{
Complex Bogie Modeling Incorporating Advanced Friction Wedge Components
}

\author{
By
}

Brian James Sperry

Thesis submitted to the faculty of the Virginia Polytechnic Institute and State University in partial fulfillment of the requirements for the degree of

Masters of Science
In
Mechanical Engineering

Dr. Corina Sandu, Chair

Dr. Mehdi Ahmadian

Dr. Robert West

May 11, 2009
Blacksburg, VA

Keywords:

Friction wedge, bogie, railway trucks, dynamic model, multibody, NUCARS ${ }^{\circledR}$

(C) Copyright 2009

Brian J. Sperry 


\title{
Complex Bogie Modeling Incorporating Advanced Friction Wedge Components
}

\author{
Brian James Sperry
}

\section{ABSTRACT}

The design of the freight train truck has gone relatively unchanged over the past 150 years. There has been relatively little change to the fundamental railway truck design because of the challenges of implementing a cost effective and reliable modification to designs that have proven effective in decades of operation. A common U. S. railway truck consists of two sideframes, a bolster, two spring nests, and four friction wedges. The two sideframes sit on the axels. The bolster rides on springs on top of the sideframes. The friction wedges also ride on springs on top of the sideframe, and are positioned between the bolster and sideframe, acting as a damping mechanism. Better understanding the dynamic behavior and forces on the bodies are critical in reducing unnecessary wear on the components, along with potential negative behavior such as loss of productivity and increase in operating costs.

This thesis will investigate the dynamic behavior of the truck under warping conditions using a stand-alone model created in Virtual.Lab. This research covers two main areas.

First, the full-truck model will be developed and its simulation results will be compared to test data from the Transportation Technology Center, Inc. (TTCI). Data was provided from warp testing performed at the $\mathrm{TTCl}$ facilities in the spring of 2008. Once validated, the model will be used to gain a better understanding of the forces and moments that are propagated through the system, and of the dynamics of all bodies. Due to costs and physical constraints, not every bogie component can be instrumented during test, so the computer model will be able to provide valuable information not easily obtained otherwise. 
Second, full-truck models using different contact geometry between the wedges, sideframes, and bolster will be compared. A model with extremely worn sideframes will allow for investigation into the effects of wear on the damping abilities and warp stiffness of the truck. Another model using split wedges will be compared with the previous model to investigate into the behavior differences in the truck using different types of wedges. By understanding the impact of different geometries on the overall performance of the truck, better decisions on design and maintenance can be made in the future.

After creating the models, we found that the full-truck model created in LMS $^{\circledR}$ Virtual.Lab compared well with the test data collected by TTCI. In the comparison with NUCARS $^{\circledR}$ we determined that the stand-alone model, which incorporates the wedges as bodies, captures the warp dynamics of the truck better than NUCARS ${ }^{\circledR}$, which models the wedges as connections. By creating a model with severely worn sideframes, we were able to determine that the truck loses its abilities to damp bounce in the system as well as to prevent warping when the components become sufficiently worn. The split-wedge model behaved similarly to the standard full-truck model for bounce inputs, but had a significantly different behavior in warp. Further development will be needed on the split-wedge model to be confident that it behaved as expected. 


\section{Acknowledgements}

I would to thank my advisor Dr. Sandu for her support during this research and throughout grad school. I would like to thank AAR for sponsoring the project. I would also like to thank Nick Wilson and Curt Urban of TTCI for all of their help with the NUCARS ${ }^{\circledR}$ simulations and for making data available to me whenever I needed it. Their knowledge was also invaluable while I attempted to create the stand-alone models. I would also like to thank my committee members Dr. Ahmadian and Dr. West for their support.

I would lastly like to thank my family for supporting me in everything I do. Without them I would not have made it to where I am today, and none of this would be possible. 


\section{Contents}

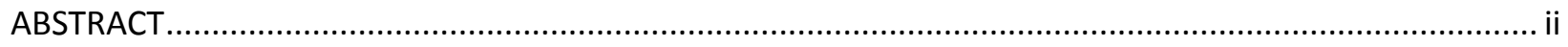

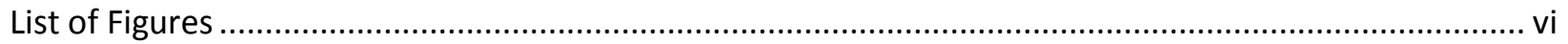

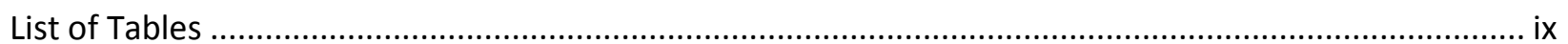

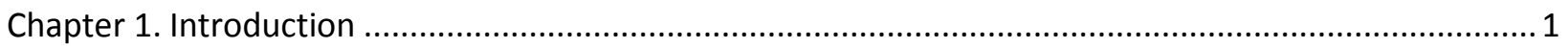

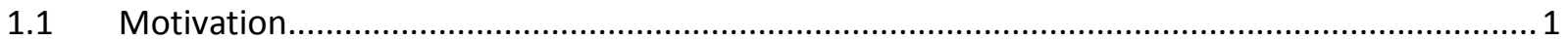

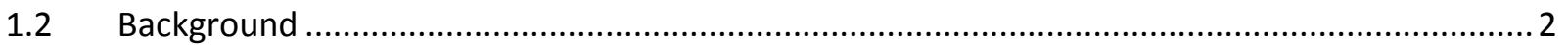

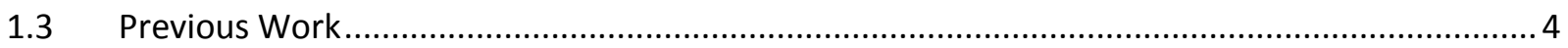

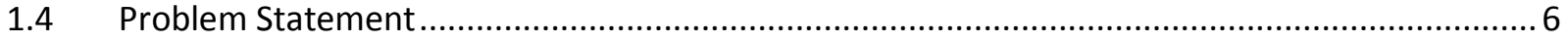

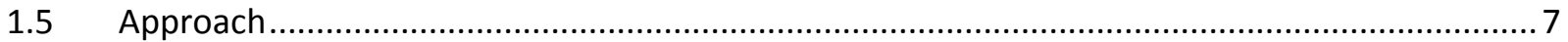

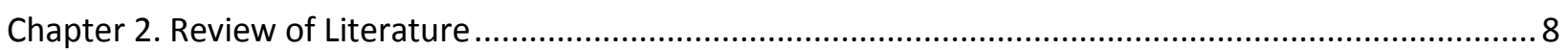

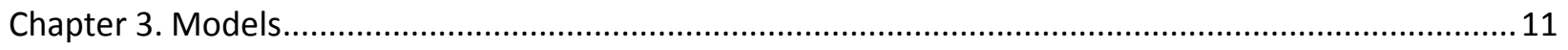

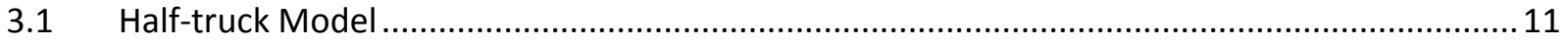

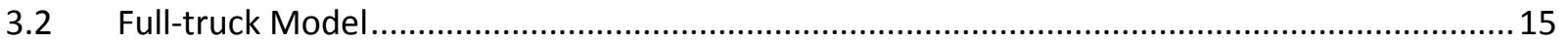

3.3 Full-truck Model with Worn Sideframes............................................................................. 19

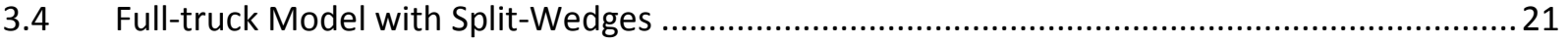

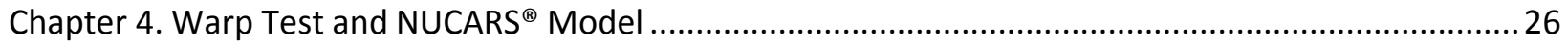

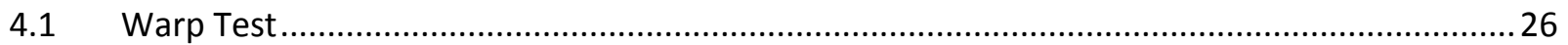

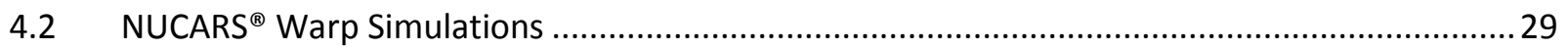

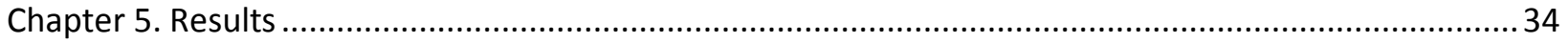

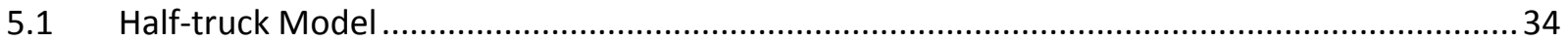

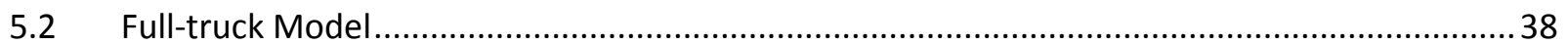

5.3 Full-truck Model with Worn Sideframes............................................................................. 54

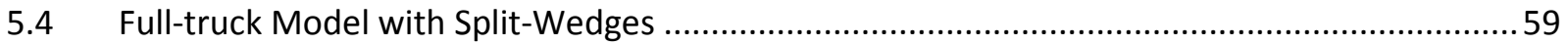

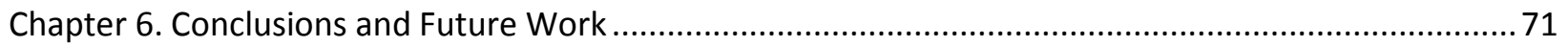

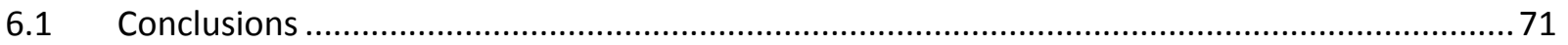

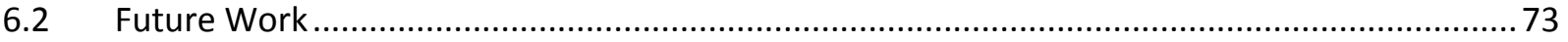

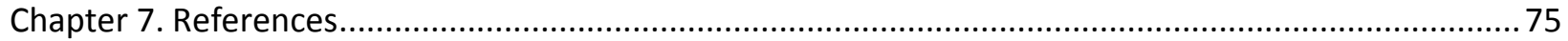




\section{$\underline{\text { List of Figures }}$}

Figure 1. Common freight car truck assembly [1] .......................................................... 3

Figure 2. Quarter-truck models developed at Virginia Tech [2] ........................................... 5

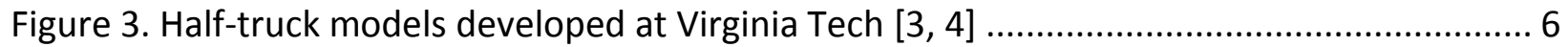

Figure 4. Half-truck model created in $\mathrm{LMS}^{\circledR}$ Virtual.Lab..................................................... 11

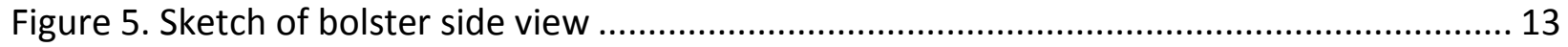

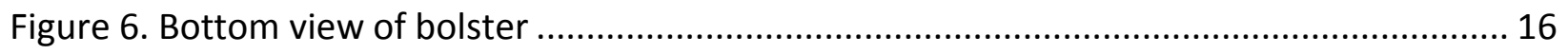

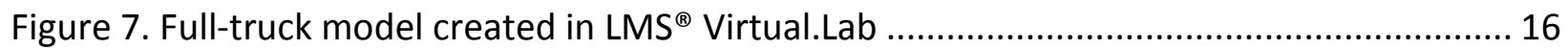

Figure 8. Schematic diagram of the full-truck model .................................................... 17

Figure 9. Diagram of the spring configuration used in the LMS ${ }^{\circledR}$ Virtual.Lab model .................. 19

Figure 10. Full-truck model with worn sideframes created in LMS ${ }^{\circledR}$ Virtual.Lab ....................... 20

Figure 11. Side view of the worn sideframe model ............................................................ 21

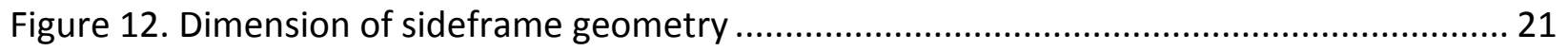

Figure 13. Comparison between a standard friction wedge and a split wedge [10] .................. 22

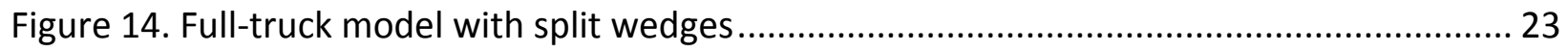

Figure 15. Split-wedge pair as modeled in $\mathrm{LMS}^{\circledR}$ Virtual.Lab .................................................. 24

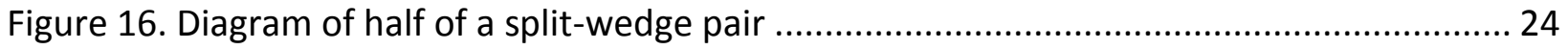

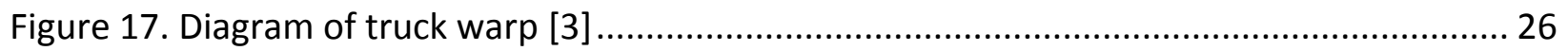

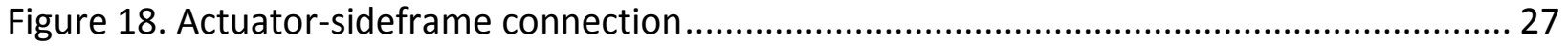

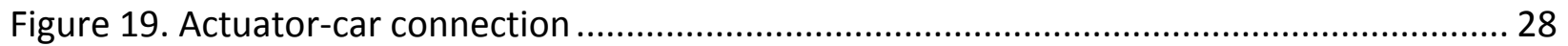

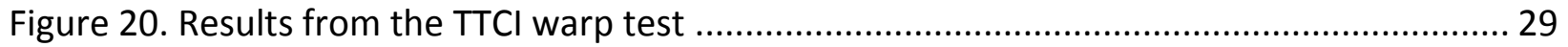

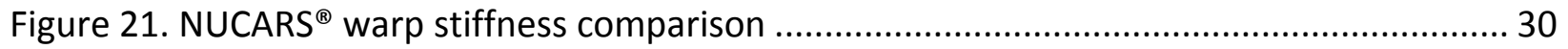

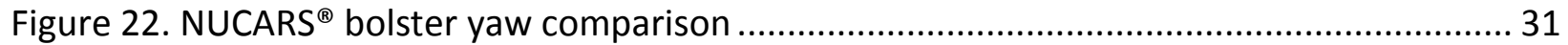

Figure 23. $\mathrm{NUCARS}^{\circledR}$ wedge vertical force comparison ..................................................... 32

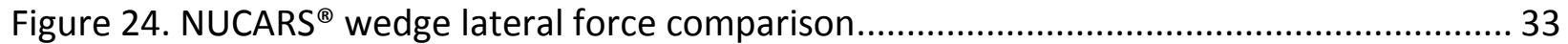

Figure 25. Bodies settling positions for the no-toe geometry case......................................... 34

Figure 26. Sideframe and bolster displacements for a 0.5 inch, $2.5 \mathrm{~Hz}$ input .......................... 35

Figure 27. Vertical wedge force hysteresis loops for all toe cases ........................................... 36

Figure 28. Vertical forces on the wedge from the $\mathrm{LMS}^{\circledR}$ Virtual.Lab model ............................. 37 
Figure 29. Vertical forces on the wedge from the MATLAB ${ }^{\circledR}$ model 37

Figure 30. Bodies settling positions for the full-truck model . 38

Figure 31. Vertical wedge force comparison between the full-truck and the half-truck models 39 Figure 32. Vertical displacement during bounce simulation 40

Figure 33. Pitch angle of the friction wedges during bounce simulation 40

Figure 34. Vertical force between wedge 1 and the sideframe 41

Figure 35. Longitudinal force between wedge 1 and the sideframe 41

Figure 36. Warp stiffness comparison between $\mathrm{LMS}^{\circledR}$ Virtual.Lab model and test data 42

Figure 37. Warp stiffness comparison between LMS $^{\circledR}$ Virtual.Lab model and NUCARS ${ }^{\circledR}$. 43

Figure 38. Bolster yaw comparison between LMS $^{\circledR}$ Virtual.Lab model and NUCARS ${ }^{\circledR}$ 44

Figure 39. Vertical wedge force comparison between $\mathrm{LMS}^{\circledR}$ Virtual.Lab model and NUCARS ${ }^{\circledR} . .45$

Figure 40. Lateral wedge force comparison between LMS ${ }^{\circledR}$ Virtual.Lab model and NUCARS ${ }^{\circledR} \ldots 46$

Figure 41. Longitudinal force comparison between LMS $^{\circledR}$ Virtual.Lab model and NUCARS ${ }^{\circledR}$...... 47

Figure 42. Bolster vertical displacement comparison between pure warp, pure bounce, and warp with bounce. 48

Figure 43. Warp stiffness comparison between pure warp and warp/bounce 49

Figure 44. Bolster yaw comparison between pure warp and warp/bounce 49

Figure 45. Wedge vertical force comparison between pure warp and warp/bounce ............... 50

Figure 46. Wedge vertical force comparison between pure bounce and warp/bounce 51

Figure 47. Longitudinal force comparison between pure warp and warp/bounce ................... 52

Figure 48. Longitudinal force comparison between pure bounce and warp/bounce................. 53

Figure 49. Bodies settling positions for the full-truck model with worn sideframes .................. 54

Figure 50. Displacement comparison between standard and worn models (bounce) ............... 55

Figure 51. Wedge pitch comparison between standard and worn models (bounce) ................. 55

Figure 52. Vertical force comparison between standard and worn models (bounce)................ 56

Figure 53. Longitudinal force comparison between standard and worn models (bounce) ......... 56

Figure 54. Warp stiffness comparison between standard and worn models (warp) .................. 57

Figure 55. Bolster yaw comparison between standard and worn models (warp) .................... 57

Figure 56. Vertical force comparison between standard and worn models (warp) .................. 58 
Figure 57. Longitudinal force comparison between standard and worn models (warp) ............ 58

Figure 58. Bodies settling positions for the full-truck with split-wedges model...................... 59

Figure 59. Displacement comparison between standard and slit-wedge models (bounce)........ 60

Figure 60. Pitch comparison between standard and split-wedge models .............................. 60

Figure 61. Vertical force comparison between standard and split-wedge models.................... 61

Figure 62. Longitudinal force comparison between standard and split-wedge models ............. 61

Figure 63. Warp stiffness comparison between standard and split-wedge models (warp) ........ 62

Figure 64. Bolster yaw comparison between standard and split-wedge models ......................63

Figure 65. Vertical force comparison between standard and split-wedge models.................... 63

Figure 66. Longitudinal force comparison between standard and split-wedge models ............. 64

Figure 67. Bolster displacement comparison between pure bounce, pure warp, and warp with

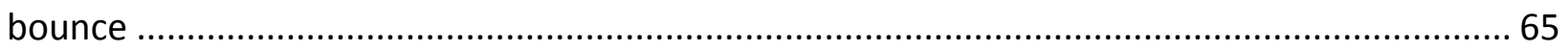

Figure 68. Warp stiffness comparison between pure warp and warp/bounce ....................... 66

Figure 69. Bolster yaw comparison between pure warp and warp/bounce............................ 66

Figure 70. Vertical force comparison between pure warp and warp/bounce ......................... 67

Figure 71. Vertical force comparison between pure bounce and warp/bounce ...................... 68

Figure 72. Longitudinal force comparison between pure warp and warp/bounce ................... 69

Figure 73. Longitudinal force comparison between pure bounce and warp/bounce................. 70 


\section{List of Tables}

Table 1. Wedge and bolster properties for the half-truck model ........................................... 14

Table 2. Contact and spring parameters for the half-truck model...................................... 15

Table 3. Wedge and bolster properties for the full-truck model ............................................ 18

Table 4. Contact and spring parameters for the full-truck model ......................................... 19

Table 5. Wedge properties for the split wedge model ........................................................... 25 


\section{Chapter 1. Introduction}

This thesis will investigate the dynamic behavior of freight train suspension, particularly friction wedges, under warping conditions. In Section 1.1, the motivation behind the research will be discussed. Section 1.2 discusses background information about the truck design for freight train cars. In Section 1.3, previous work that has led to the current research will be discussed. Section 1.4 explains the problem statements of this work. Lastly, Section 1.5 describes the research approach used in this study.

\subsection{Motivation}

There are a few software programs used to simulate the behavior of freight trains, such as NUCARS ${ }^{\circledR}$ and VAMPIRE $^{{ }^{2}}$. While such programs are able to capture much of the dynamic behavior of freight cars, the method of modeling the wedge simply as a force equation rather than a body may encounter situations where the behavior of the bogie cannot be modeled accurately. The wedge mass and inertial properties are traditionally ignored because they are so small compared to the other train components, that they are considered negligible. Due to this very small mass compared to the other components, high frequency noise is also a very common problem in simulations. For these reasons, an equation that relates the load to the damping force is used. While these equations have become more sophisticated over time, and now are able to output more of the relevant information, there are still situations that these equations fail to capture.

The stand-alone model developed in this study incorporates the friction wedges as bodies rather than modeling their effects using just an equation. One of the important capabilities that this gives us is the ability to see how the moments propagate through the different bodies in the system. While the mass and inertia of the wedge may be negligible, the affects of the geometry of the solid body is not. A prime example of this is the study of the fulltruck under a warping condition, where one sideframe is pushing forward and the other is

\footnotetext{
${ }^{1}$ NUCARS $^{\circledR}$ is a registered trademark of the Transportation Technology Center, Inc. (TTCI), a wholly owned subsidiary of the Association of American Railroads (AAR)

${ }^{2}$ VAMPIRE ${ }^{\circledR}$ is a U.K. registered trademark of DeltaRail Group Ltd.
} 
pushing back. In this case, the shape of the wedge creates a stiffness, which tries to keep the truck square. This warping stiffness is greatly affected by the shape of the wedge, and the contact conditions between the sideframe or the bolster and the wedge. Without the components of the truck system being properly modeled, these affects are lost. This is problematic, because the ability to model warping behavior is very important to understanding the overall dynamics of the truck, as it is a situation that can lead to dangerous conditions, such as derailment.

Due to its ability to model the forces under the warping condition, the stand-alone fulltruck model can give a researcher valuable information about the truck dynamics. Another capability of the model is that simulations can be run for different wear levels and configurations in the components. By monitoring the warp stiffness of different configurations, decisions could be made as to when the stiffness falls below an acceptable level, which could improve the preventative maintenance on freight train trucks.

\subsection{Background}

The current truck assembly on freight train cars has been used, relatively unchanged, for the past 150 years. Figure 1 shows a common freight car truck assembly. The simplicity of the truck, which is held together purely by gravity and the weight of the components, has proven to work very well over the years. The base of the truck is composed of the two axles ( 1 and 2 ) and wheels, which are in contact with the rails. Sitting on top of the axles are two sideframes ( 3 and 4); one on each side running parallel to the tracks. A spring nest (5) is situated on each sideframe, which consists of a number of springs which carry the bolster (6) and friction wedges. The bolster lays across the two sideframes, perpendicular to the tracks. The friction wedges sit in between the bolster and the sideframe, one at each corner of the bolster. The car is connected to the truck by sitting on the bolster, with a pivot connection that allows rotation between them. 


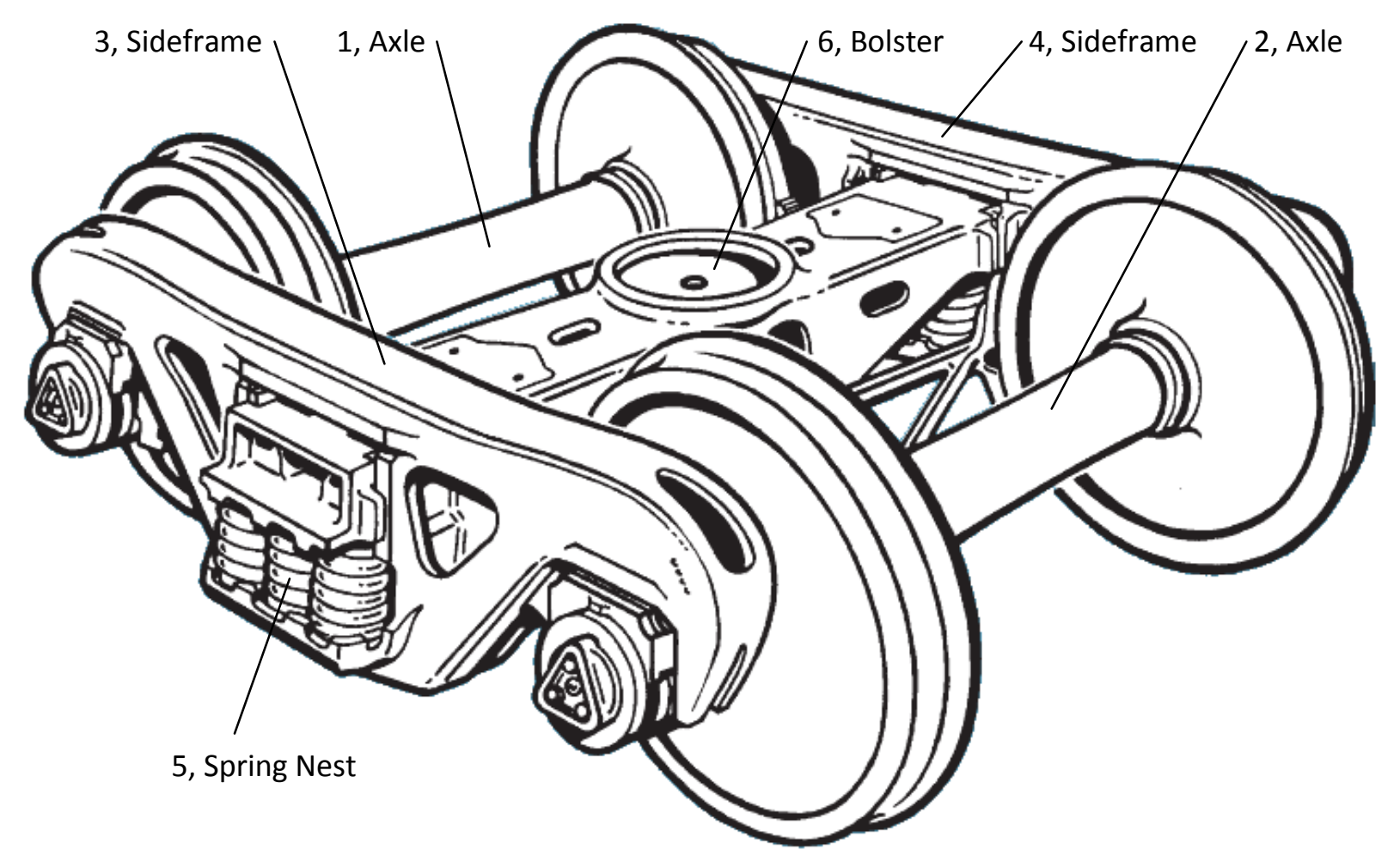

Figure 1. Common freight car truck assembly [1]

The friction wedge is used instead of a more conventional hydraulic damper in order to reduce maintenance time and expense. The weight of freight cars puts immense loads and stresses on all of the parts in the truck. By reducing moving parts, and keeping them simple, the maintenance can be completed more quickly, which is critical in a system where many trains are sharing the same track. A single on-track breakdown or derailment can cause significant delays if the problem is not dealt with in a very timely manner.

Under normal operating conditions, the friction wedges gradually become worn, slowly diminishing their damping ability. As the wedges, sideframes, or bolsters wear, the wedge is allowed to rise with respect to the bolster. Since the damping force is reliant on the force exerted by the spring on which it sits, the less compression in the spring, the lower the damping force will be. Another issue that can be detrimental to the performance of the truck with worn friction wedges is the greater possibility of the wedge getting stuck between the sideframe and 
bolster. This can become a very dangerous situation, because it effectively locks the entire suspension system, making the connection between the car and the axles rigid. Without a working suspension system, the impact forces become much greater, increasing the risk of more serious damage to the truck, the car, and the cargo.

Warping of the truck is another behavior seen in freight train trucks that can create an unsafe condition. While a small amount of warping can help the truck go around a turn better, the ideal truck would not allow warping on straight track. Two very serious issues caused by warping on straight track are track spreading and truck hunting. While the truck is warped, the front wheel on one side and the back wheel on the other side are pushed out against the track. Over time this force can spread the track. Hunting occurs when due to warping the truck begins weaving from one side of the track to the other. This also creates large forces between the wheels and the track, which can spread the track. If hunting becomes severe enough, the shifting weight can also lead to derailments. For these reasons, a truck with high warp stiffness is desirable, as it would be more capable of preventing truck warp.

The safety and financial costs involved make it critical to know when a truck can no longer operate safely. By creating a full-truck model with all of the major components modeled as bodies, the goal is to get a better understanding of the dynamics of the entire system, and of the interactions between the bodies involved. Simulations of different sideframe inputs and part geometries will allow researchers to visualize and investigate dynamic effects in the truck, and raise flags that will help prevent future failures, improve the truck design, and maintain safe operating conditions.

\subsection{Previous Work}

Virginia Tech's Railway Technologies Laboratory (RTL) began researching friction wedge behavior as an Association of American Railroads (AAR) affiliated lab. Initially the existing wedge models in NUCARS $^{\circledR}$ were evaluated using various inputs. The research was then extended to creating a stand-alone quarter-truck model to be compared with the NUCARS ${ }^{\circledR}$ 
models. This stand-alone model was created using MATLAB ${ }^{{ }^{3}}$ and Mambo, a freeware dynamics program. The quarter-truck model consisted of a sideframe, control coils, a quarter of a bolster, and a friction wedge, with bolster inputs. The quarter-truck model was created with both variably damped and constantly damped geometry. Figure 2 shows the quarter-truck models developed in the RTL at Virginia Tech [2].
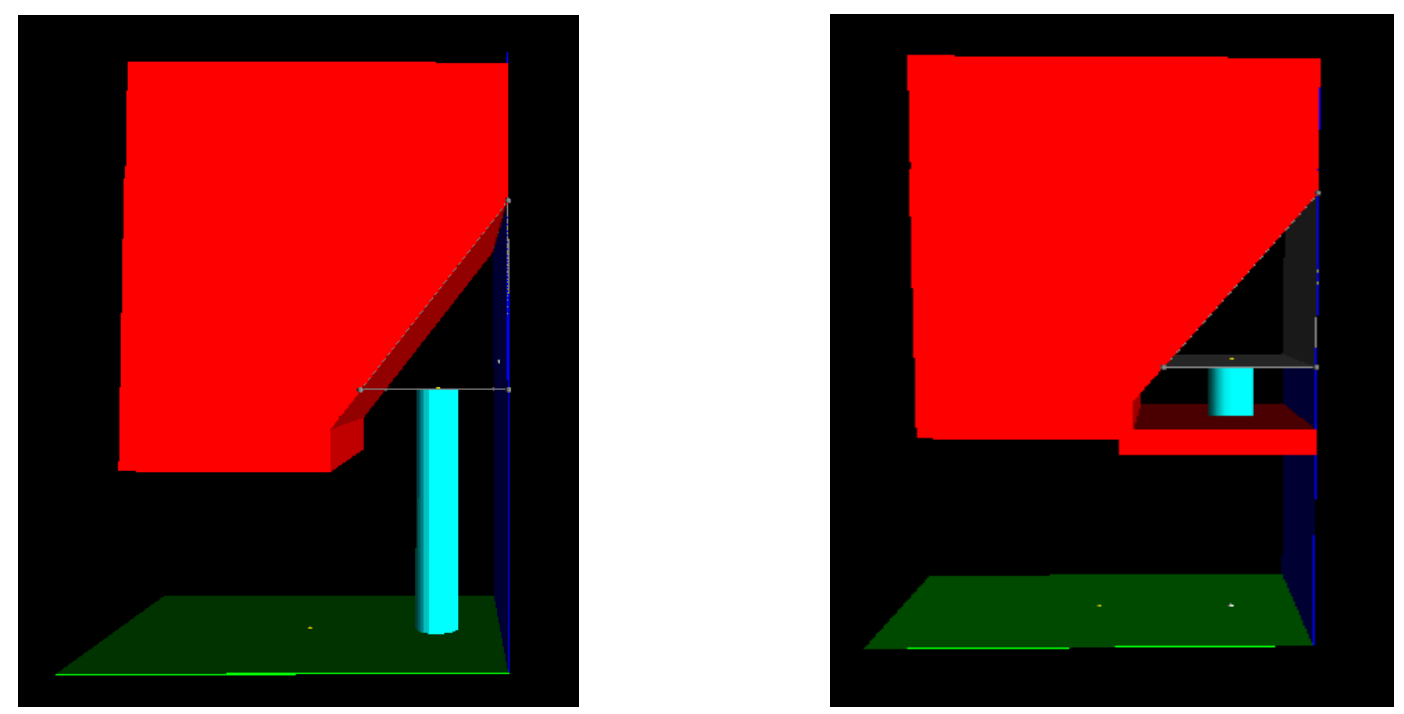

Figure 2. Quarter-truck models developed at Virginia Tech [2]

The stand-alone model was later expanded to a half-truck model, which incorporated a sideframe, control coils, half of a bolster, and two friction wedges. The input body was changed to be the sideframe, to better simulate real world inputs. Again, variably damped and constantly damped geometries were created. The data from simulations in the stand-alone model were again compared with $\mathrm{NUCARS}^{\circledR}$ outputs. Figure 3 shows the half-truck models developed at Virginia Tech $[3,4]$.

\footnotetext{
${ }^{3}$ MATLAB is a registered trademark The MathWorks
} 

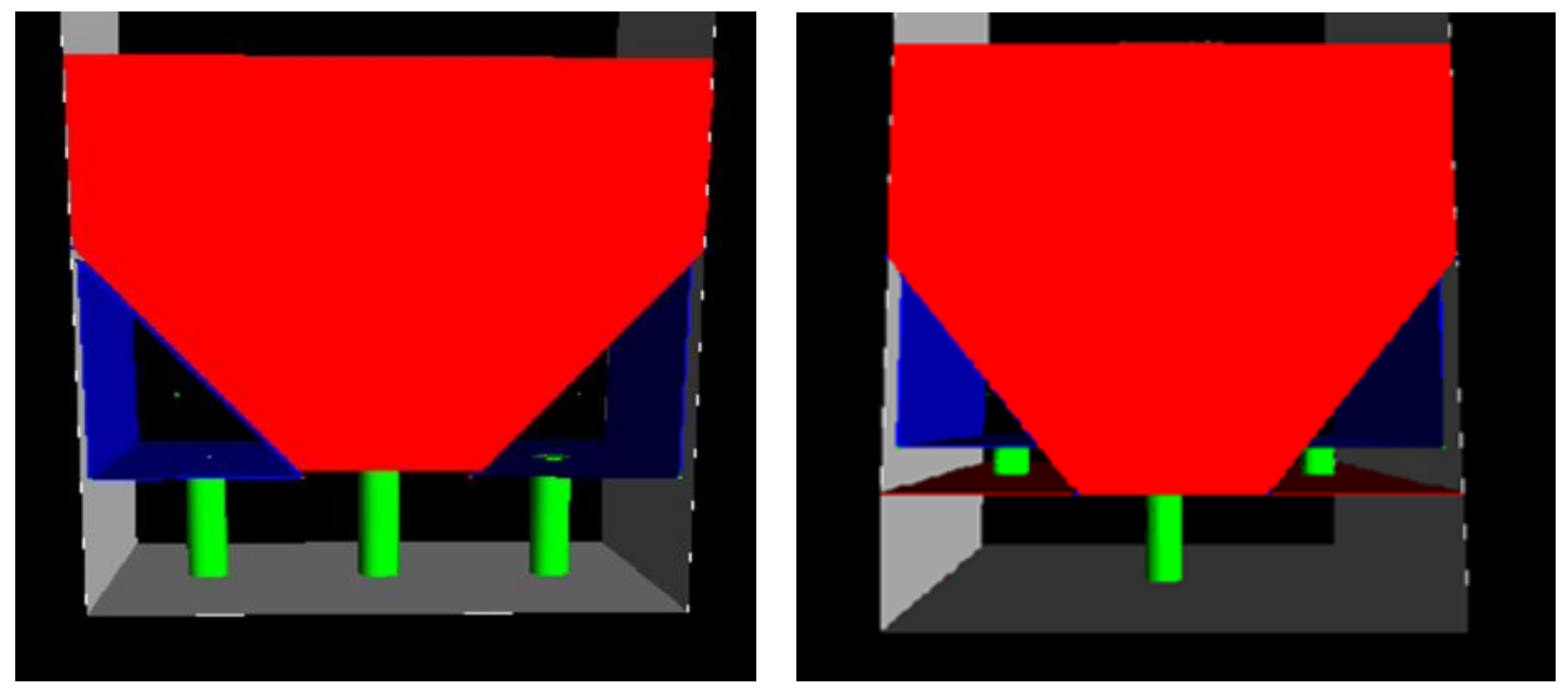

Figure 3. Half-truck models developed at Virginia Tech [3, 4]

The stand-alone models showed significant differences than NUCARS ${ }^{\circledR}$ in many parameters that were output. While there was no experimental data to compare to at the time, it is believed that the stand-alone model was capturing events that $\mathrm{NUCARS}^{\circledR}$ was not capable of, because of the way the wedges are modeled as a 'joint's' without geometry or inertial properties.

The research discussed in this thesis is a continuation of this work that has been ongoing at the RTL.

\subsection{Problem Statement}

The purpose of this research is to gain a better understanding of the dynamic behavior of the freight train truck, focusing especially on the friction wedge's contribution to the behavior. By developing a better understanding of the importance of each of the bodies within the truck, future simulation software will better capture truck behavior, and therefore, overall car behavior.

The first goal of this research was to create a full-truck model that incorporated each component of the truck, with appropriate body dimensions, mass, and inertial properties. 
Simulation results of this model will be compared with warp test data performed at TTCI, and with NUCARS ${ }^{\circledR}$.

The second goal was to create a full-truck model with severe wear on the sideframes. The output data from this model will be compared with the original model to evaluate the effects of wear on the warp performance of the truck.

The third goal was to create a full-truck model with split-wedges instead of standard (one piece) friction wedges. The data obtained from this model will also be compared with the original model to evaluate the warp performance of the split-wedge, since there is currently no test data available for dynamics of a truck with split-wedges.

\subsection{Approach}

Each of the full-truck models considered will be developed in LMS $^{\circledR}$ Virtual.Lab, a widely used, general multibody dynamics modeling software. Input data and the geometry parameters will be provided from $\mathrm{TTCl}$, and were used to create a model that accurately represents the full-truck used in the warp testing that was performed at $\mathrm{TTCl}$ in the spring of 2008. Using this data, the model of the truck will be created from the sideframes up. This setup includes the two sideframes, two full spring nests, four friction wedges, and a bolster.

The two sideframes will be created as massless rigid bodies. The sideframes will be left massless because they will be used as the input bodies, so their mass will never oppose any motion within the system. The friction wedges will be created with the geometry, mass, and inertial properties of an actual wedge. The bolster will be given the inertial properties of a real world bolster, with the mass of the car also lumped at the bolster. The wedges and the bolster will be connected to the sideframe by a full spring nest. The contact with the sideframe and with the bolster will be modeled using a five-by-five grid of contact points on each face of the friction wedges. 


\section{Chapter 2. Review of Literature}

This chapter will introduce reference material that was reviewed before research began.

\section{Advanced Multibody Dynamics Modeling of the Freight Train Truck System [3] by B. Ballew}

This paper is a continuation of research from the Railway Technology Laboratory at Virginia Tech. The study's focus was to create a stand-alone model which could capture the dynamic behavior of the friction wedge. A half-truck model was created in MATLAB ${ }^{\circledR}$ to include four rigid bodies: a bolster, a sideframe, and two friction wedges. The model included geometries and inertial properties of the friction wedges, and allowed them four degrees-offreedom: vertical and longitudinal displacement, pitch, and yaw. Models were created for both variably damped and constantly damped configurations, and results were compared with NUCARS $^{\circledR}$. This paper also discusses the implementation of wedge wear as an input, by creating a quarter-truck variably damped model that allows for curved wedge faces.

A Multibody Dynamics Approach to the Modeling of Friction Wedge Elements for Freight Train Suspensions [2] by J. Steets

This paper investigates modeling the interactive effects of the friction wedge with the bolster and sideframe. A quarter-truck model was created using MATLAB ${ }^{\circledR}$, which gave the friction wedge geometry and inertial properties. This model was compared with NUCARS ${ }^{\circledR}$, and was shown to be capable of capturing events with NUCARS ${ }^{\circledR}$ could not.

A Multibody Dynamics Approach to the Modeling of Friction Wedge Elements for Freight Train Suspensions: Part I - Theoretical Modeling [5] by J. Steets, B. Chan, and C. Sandu

This paper presents the theoretical approach to producing a stand-alone model which will better capture friction wedge interactions with the bolster and sideframe. This approach uses a fully defined wedge with four degrees-of-freedom, rather than the simple damping equations used by many train modeling software's. 
A Multibody Approach to the Modeling of Friction Wedge Elements for Freight Train Suspension: Part II - Simulation and Analysis [6] by J. Steets, B. Chan, C. Sandu, B. Ballew

This paper presents the results of the previous papers modeling approach. The results of the stand-alone MATLAB ${ }^{\circledR}$ model were compared with results of NUCARS ${ }^{\circledR}$ simulations with similar inputs. These comparisons showed improvements in capturing wedge behavior, and were able to capture parameters which NUCARS ${ }^{\circledR}$ could not.

Modeling Friction Wedges, Part I: The State-of-the-Art [7] by P. Klauser

This paper discusses how current software models the friction wedge element. The author discusses how both $\mathrm{NUCARS}^{\circledR}$ and $\mathrm{VAMPIRE}^{\circledR}$ model the friction wedge as a two dimensional force, with vertical and lateral translations. The author then compares how the two software's systems compare with each other, and what improvements could be made to how the friction wedge is modeled.

Modeling Friction Wedges, Part II: The State-of-the-Art by [8] P. Klauser

This paper discusses the implementation of a new friction wedge model in VAMPIRE ${ }^{\circledR}$. The new model included mass for the friction wedges, which had been seen as insignificant in previous iterations. The model also added a grid of elements across the faces of the friction wedges which give a pressure distribution across the faces and allow for more accurate warp stiffness calculations.

Dynamic Modeling and Simulation of Three-Piece North American Freight Vehicle Suspension with Non-linear Frictional Behavior Using ADAMS/Rail [9] by R. Harder

This paper presents the methods they used to create a friction wedge and three-piece bogie model using ADAMS. The author discusses the results of this model, and the challenges of creating a model due to the complexity of the behavior of the friction wedge.

Modeling and Dynamics of Friction Wedge Dampers in Railroad Freight Trucks [10] by A. Kiser, J. Cusumano, and J. Gardner

This paper presents a model that was developed of the bolster and friction wedge elements, using dry friction formula for contact between bodies. Their model showed that the friction behavior varied significantly with different amplitudes and speed. It was determined 
that models that use equivalent linear models for the behavior of the friction wedge may be unable to accurately model many important cases.

After reviewing literature related to freight train suspension modeling, it was found that the current systems being use do not adequately model the behavior of the system for some common and important scenarios. While the friction wedge models have been improving, it is still common practice to neglect the geometry and inertial properties of the wedge. Research has shown that including the geometry and inertia of the wedges, the model can better capture the behavior of the system. 


\section{Chapter 3. Models}

This chapter of the thesis explains the different stand-alone models that were developed using LMS $^{\circledR}$ Virtual.Lab. Section 3.1 describes the half-truck model created for validating the implementation of the LMS $^{\circledR}$ Virtual.Lab model against the MATLAB ${ }^{\circledR}$ model [3]. Section 3.2 focuses on the development of the full-truck model which would be used for warp simulations. Section 3.3 highlights the changes in the full-truck model to incorporate worn sideframes and wedges. Section 3.4 explains the split-wedge model which was made to investigate the effects of different types of wedge geometry.

\subsection{Half-truck Model}

The first step of developing the full-truck models was to develop a half-truck model for comparison with the models discussed in the Previous Work section. Figure 4 shows the halftruck model created in $\mathrm{LMS}^{\circledR}$ Virtual.Lab.
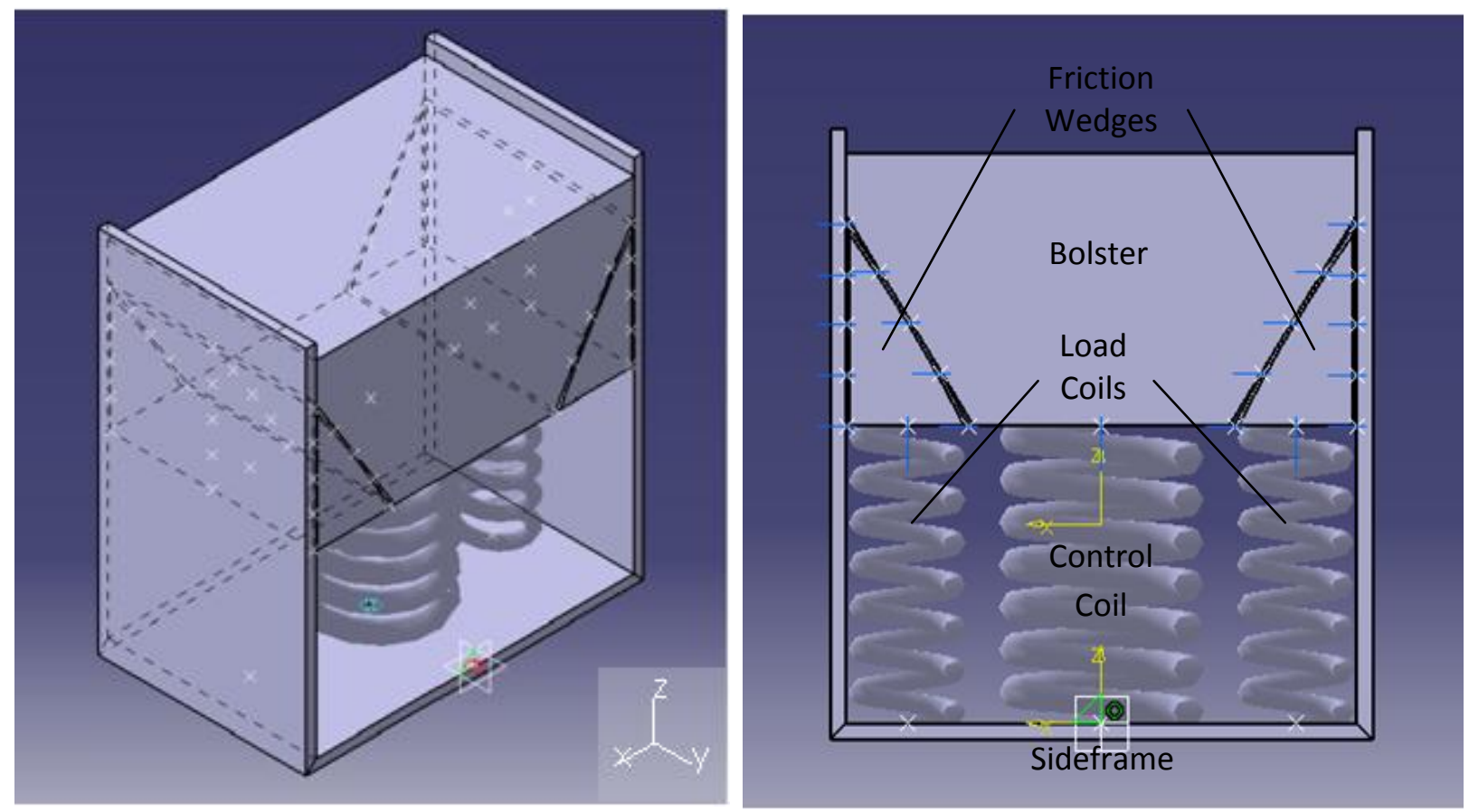

Figure 4. Half-truck model created in $\mathrm{LMS}^{\circledR}$ Virtual.Lab

It was important to keep some level of continuity with the previous work done at Virginia Tech, which made this comparison necessary. It was also important to see that the 
results were similar, because there was no experimental data available for validation at the time, so the best comparison possible was against these previous models. Moreover, the fulltruck model is able to predict dynamic effects for which there is no experimental data available; thus, it was important to gain confidence in the modeling technique by first validating the halftruck model against accepted results.

The main analysis in this study focused on warping simulations, for which the test data came from a car with a constantly damped suspension system. Because the full-truck model had to have a constantly damped configuration, this was implemented in of the half-truck model as well. The basic parameters of the model were created to match the previous MATLAB ${ }^{\circledR}$ model [3].

The half-truck model consists of 4 bodies: the sideframe, the bolster, and two friction wedges. The bodies were created to closely approximate the geometries of the actual parts; in the case of the bolster, just the portion of the bolster in contact with the wedges has accurate geometry implemented. The sideframe was created in LMS ${ }^{\circledR}$ Virtual.Lab by first creating a " $U$ "shaped line using the sketch function that was the basic shape of the part of the sideframe that was of interested for the simulations. This sketch was then extruded to give the sideframe a depth dimension. Finally, the thick surface command was used to give the body thickness, which was necessary for it to be used in conjunction with contact points. The wedge was modeled with a curved face in contact with the bolster, which would be the geometry in a new wedge. To create the wedge, a sketch of the outline of the side view of the wedge was created, and then extruded to the appropriate depth. The bolster was created in the same manner as the wedges, by first creating a sketch of the side view of the bolster, and then extruding the body to the appropriate depth. Figure 5 show the dimensions of the main bolster body. The parameters of the bodies are listed in Table 1. 


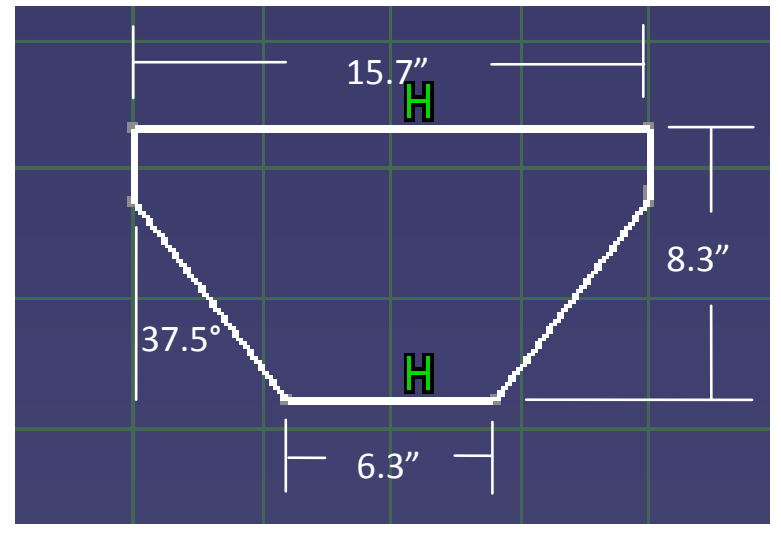

Figure 5. Sketch of bolster side view

The contact between the wedges and the bolster or sideframes was modeled as a grid of point contacts. Each wedge had a grid of points, five rows and three columns, on the face in contact with the bolster and the same for the face in contact with the bolster sideframe. Each point was constrained to a particular position on the surface of one of the wedges. The sphereto-extruded surface contact type was assigned to each of these points. The parameters of contact were then entered into the sphere-to-extruded surface contact window. These parameters included the material properties of the extruded surface, which in our case was either the sideframe or the bolster. A radius for each contact point also was required; in our case we chose used a radius of 0.001 inches. The contact forces are calculated by determining the relative location of the contact points to the body it is contacting. The contact forces become active any time the distance between the sphere and the respective extruded surface is equal to or less than zero. This relationship determines the normal force on the sphere, and is used to also calculate the frictional forces on the sphere. One concession that had to be made by using point contact was that only a dynamic friction coefficient could be input, whereas full surface contact allowed both static and dynamic friction coefficients to be used.

Points were also defined on the underside of the wedges and bolster and on the sideframe for the springs. The spring forces were defined by inputting the free length and stiffness of the spring that connects the points. In this case, equivalent spring forces were used rather than a full spring nest. The parameters of the contact points and spring forces are listed in 
Table 2. As in the models previously created at Virginia Tech, the model was modified to incorporate the toe-in (-0.003 rad, wear plates slightly closer together at bottom), no-toe (0 rad, wear plates parallel), and toe-out (0.003 rad, wear plates slightly further apart at bottom) geometries. These geometries were created by slightly modifying the shape of the sketch used to create the sideframe body. These geometries were created by slightly modifying the shape of the sketch used to create the sideframe body. The top of the sideframe was held at a constant width while the bottom was narrowed or widened to achieve the appropriate angle.

Table 1. Wedge and bolster properties for the half-truck model

\begin{tabular}{|c|c|}
\hline Wedge $h_{x}$ (in) & 3.827 \\
\hline Wedge $h_{y}$ (in) & 10.5 \\
\hline Wedge $h_{z}$ (in) & 6.125 \\
\hline Wedge face curvature radius (in) & 45 \\
\hline Wedge mass (lb) & 28.15 \\
\hline Wedge $I_{x x}\left(I b-i n^{2}\right)$ & 10.775 \\
\hline Wedge $I_{y y}\left(I b-i n^{2}\right)$ & 3.803 \\
\hline Wedge $I_{z z}\left(I b-i n^{2}\right)$ & 9.107 \\
\hline Bolster mass (lb) & 65000 \\
\hline Bolster $I_{x x}\left(I b-i n^{2}\right)$ & 10443 \\
\hline Bolster $I_{y y}\left(I b-i n^{2}\right)$ & 6750.8 \\
\hline Bolster $I_{z z}\left(I b-i n^{2}\right)$ & 7494.9 \\
\hline Sideframe $h_{x}$ (in) & 16 \\
\hline Sideframe $h_{z}$ (in) & 18 \\
\hline
\end{tabular}


Table 2. Contact and spring parameters for the half-truck model

\begin{tabular}{|l|l|}
\hline Sideframe coefficient of friction $\mu_{\mathrm{s}}$ & 0.40 \\
\hline Bolster coefficient of friction $\mu_{\mathrm{b}}$ & 0.25 \\
\hline Sideframe stiffness (Ibf/in) & $10^{6}$ \\
\hline Bolster stiffness (lbf/in) & $10^{6}$ \\
\hline Control coil stiffness (lbf/in) & 1491 \\
\hline Load coil stiffness (lbf/in) & 22417.8 \\
\hline
\end{tabular}

\subsection{Full-truck Model}

The full-truck stand-alone model was developed and simulation results were compared to data from a warp test performed at $\mathrm{TTCl}$ in the spring of 2008. The model consists of seven bodies; two sideframes, four friction wedges, and a bolster. After validating the half-truck model, upgrades were made to the full-truck model. Most notably, the contact grid on the wedges was increased to a five-by-five grid, and the equivalent springs were replaced by full spring nests, as they appear in an actual truck. The process for making the bodies on for the full-truck model was the same as for the half-truck model for the sideframes and wedges. The centers of the sideframes were placed 39 inches from the center of the truck. The bolster was considerably different for the full-truck model. As with the half-truck model, a sketch of the bolster's primary shape, with the edges angled to match the wedge geometry, was created and then extruded. The bolster was extruded 47 inches in each direction so that it was able to span across the sideframes. The bottom face of the bolster was then selected, and sketches of the pocket walls were created. These sketches were then extruded into the existing bolster. Figure 6 shows a bottom view of the bolster, where the pockets are visible. Figure 7 shows the fulltruck model created in LMS $^{\circledR}$ Virtual.Lab. Figure 8 is a schematic of the full-truck model which shows how the body's interactions were modeled. The numbering convention seen in the schematic was consistent throughout all of the models and was used in the analysis. 


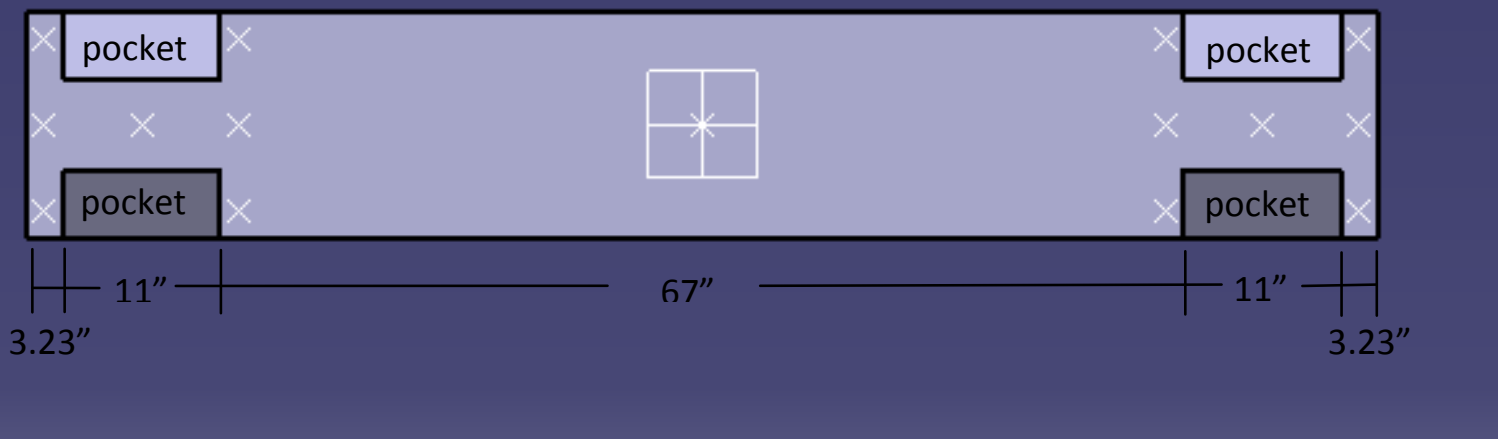

Figure 6. Bottom view of bolster

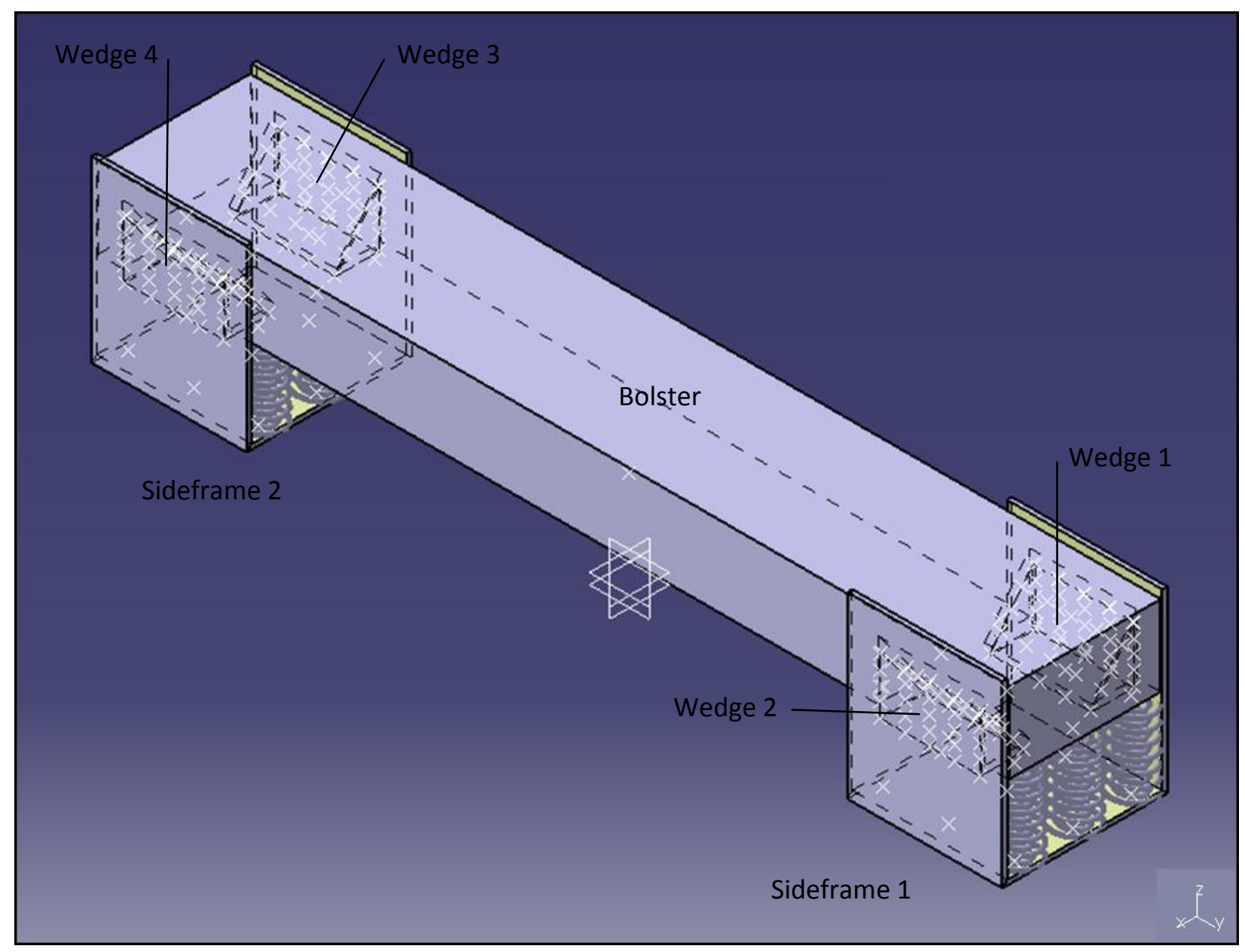

Figure 7. Full-truck model created in LMS $^{\circledR}$ Virtual.Lab 


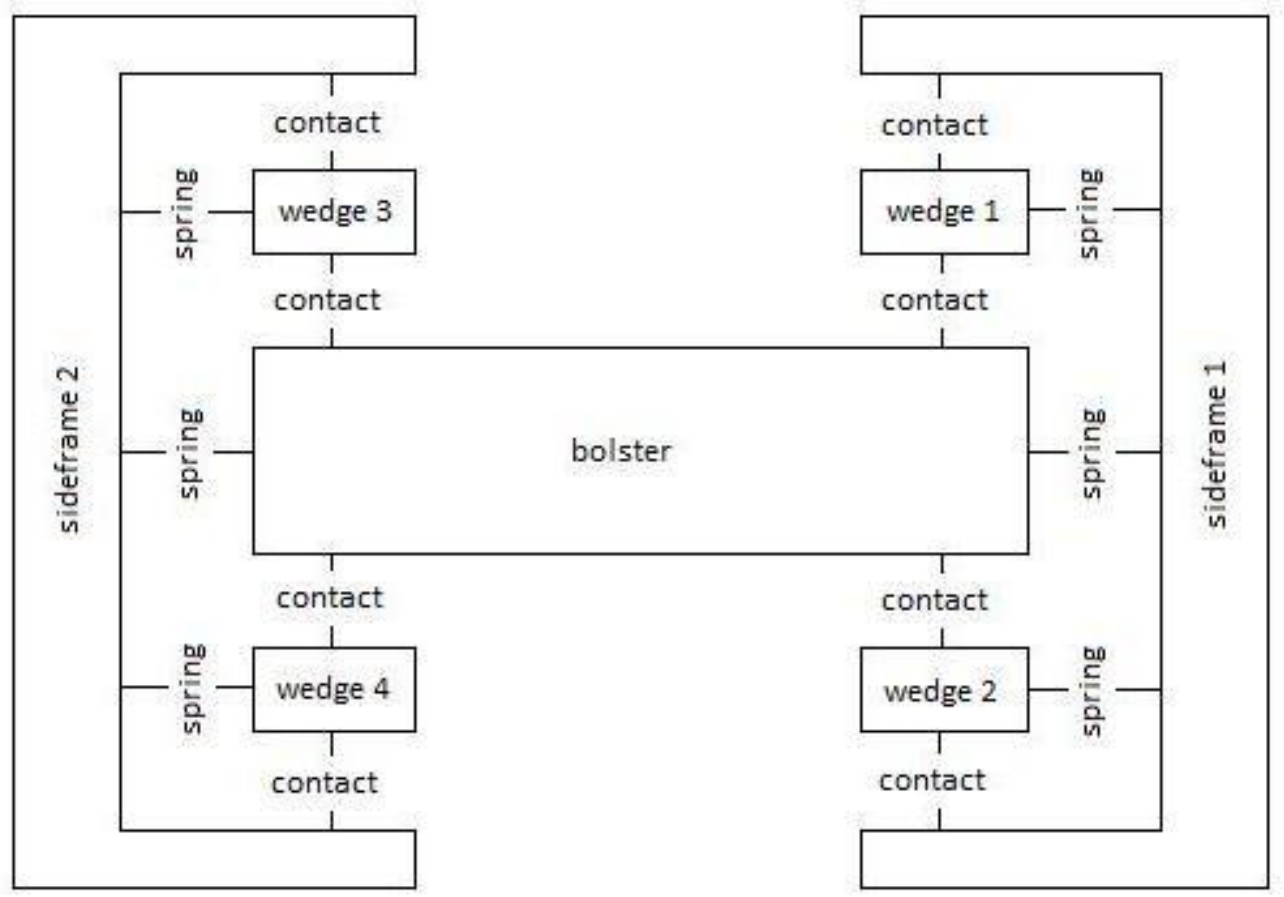

Figure 8. Schematic diagram of the full-truck model

In the full-truck model, the sideframes were used as the input bodies for motion, since this is a realistic input for the truck in a real-life situation. The sideframe was otherwise constrained so that all of the motion was limited to the $\mathrm{x}$-direction, which would be the direction of the tracks, with no rotation allowed, as it is the stable platform on which the suspension sits. The four friction wedges were allowed all six degrees of freedom, three translational and three rotational. The bolster was also given six degrees, but a pivot was placed on the bolster that effectively removed the $x$ - and $y$-translations. This was done to replicate the constraints placed on the bolster by the car and the sideframes, which were not modeled as bodies in this study. The properties of the friction wedges and bolster are listed in Table 3. 
Table 3. Wedge and bolster properties for the full-truck model

\begin{tabular}{|l|l|}
\hline Wedge $\mathrm{h}_{\mathrm{x}}(\mathrm{in})$ & 4.7 \\
\hline Wedge $\mathrm{h}_{\mathrm{y}}(\mathrm{in})$ & 10.5 \\
\hline Wedge $\mathrm{h}_{\mathrm{z}}(\mathrm{in})$ & 6.125 \\
\hline Wedge face curvature radius $(\mathrm{in})$ & 126.8 \\
\hline Wedge mass $(\mathrm{lb})$ & 28.15 \\
\hline Wedge $\mathrm{I}_{\mathrm{xx}}\left(\mathrm{lb}-\mathrm{in}^{2}\right)$ & 10.775 \\
\hline Wedge $\mathrm{I}_{\mathrm{yy}}\left(\mathrm{lb}-\mathrm{in}^{2}\right)$ & 3.803 \\
\hline Wedge $\mathrm{I}_{\mathrm{zz}}\left(\mathrm{lb}-\mathrm{in}^{2}\right)$ & 9.107 \\
\hline Bolster $\mathrm{mass}_{\mathrm{l}}(\mathrm{lb})$ & 121500 \\
\hline Bolster $\mathrm{I}_{\mathrm{xx}}\left(\mathrm{lb}-\mathrm{in}^{2}\right)$ & 20885.719 \\
\hline Bolster $\mathrm{I}_{\mathrm{yy}}\left(\mathrm{lb}-\mathrm{in}^{2}\right)$ & 13411.58 \\
\hline Bolster $\mathrm{I}_{\mathrm{zz}}\left(\mathrm{lb}-\mathrm{in}^{2}\right)$ & 14989.861 \\
\hline
\end{tabular}

For the full-truck model the moments on the bodies were of greater interest, so the full spring nest was modeled. The nest consists of 18 springs on each sideframe, in a three-bythree configuration, with each location having an inner- and outer-spring. Of these springs, 14, called the load springs, hold the majority of the weight of the bolster. The other four are used to push the wedges up against the bolster, two springs per wedge. In the full truck model the stiffness of the bolster was increased to $10^{7} \mathrm{lbf} /$ in. Three different stiffness's were tested on this model to see if they affected the warp stiffness. It was found that the results were the same with all of the bolster and sideframe stiffness's, so the middle value was chosen. Figure 9 shows the configuration of these springs. The properties of these springs and the contact properties are listed in Table 4. 


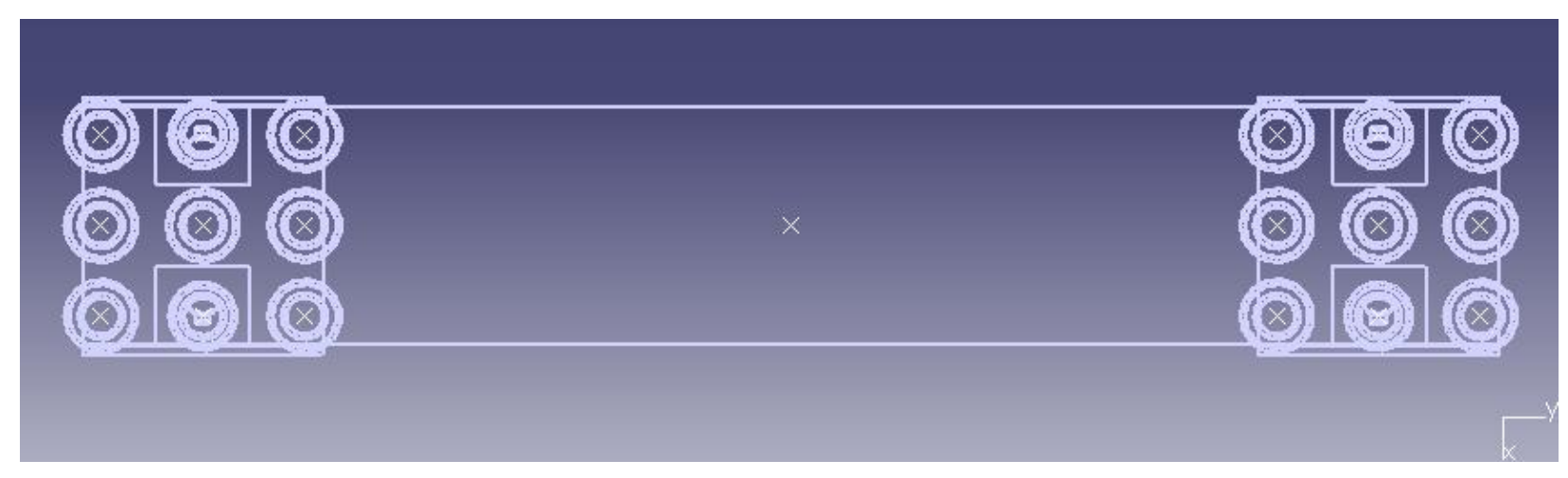

Figure 9. Diagram of the spring configuration used in the $\mathrm{LMS}^{\circledR}$ Virtual.Lab model

Table 4. Contact and spring parameters for the full-truck model

\begin{tabular}{|l|l|}
\hline Sideframe coefficient of friction $\mu_{\mathrm{s}}$ & 0.40 \\
\hline Bolster coefficient of friction $\mu_{\mathrm{b}}$ & 0.25 \\
\hline Sideframe stiffness (Ibf/in) & $10^{7}$ \\
\hline Bolster stiffness (Ibf/in) & $10^{7}$ \\
\hline Inner control coil stiffness (Ibf/in) & 480 \\
\hline Outer control coil stiffness (Ibf/in) & 996 \\
\hline Inner load coil stiffness (Ibf/in) & 1121.067 \\
\hline Outer load coil stiffness (Ibf/in) & 2241.627 \\
\hline Inner control coil length (in) & 12.19 \\
\hline Outer control coil length (in) & 12.06 \\
\hline Inner load coil length (in) & 10.313 \\
\hline Outer load coil length (in) & 10.25 \\
\hline
\end{tabular}

\subsection{Full-truck Model with Worn Sideframes}

In order to determine the effect that wear has on the warp resistance of a truck, a model was created with severely worn sideframes. Figure 10 shows the full-truck model with worn sideframes created in $\mathrm{LMS}^{\circledR}$ Virtual.Lab. Based on information provided by $\mathrm{TTCl}$, the sideframes and friction wedges were made with matching curvature, which makes the 
interaction between them similar to a revolute joint. To create the curvature of the sideframe and wedge, simple modifications were made to their respective sketches. Other than the change to the sketches, the procedures for creating the model were identical to that of the fulltruck model. Figure 11 is a side view of the worn sideframe model, in which the curvature of the sideframes and wedges can be seen. The worn sideframe model has properties which are identical to that of the original full-truck model, other than the fact that the wedge face and sideframe were given a 20 inch radius curve, which is modeled as if material were worn away from the sideframe. Figure 12 shows the dimensions of the sideframe for the worn model.

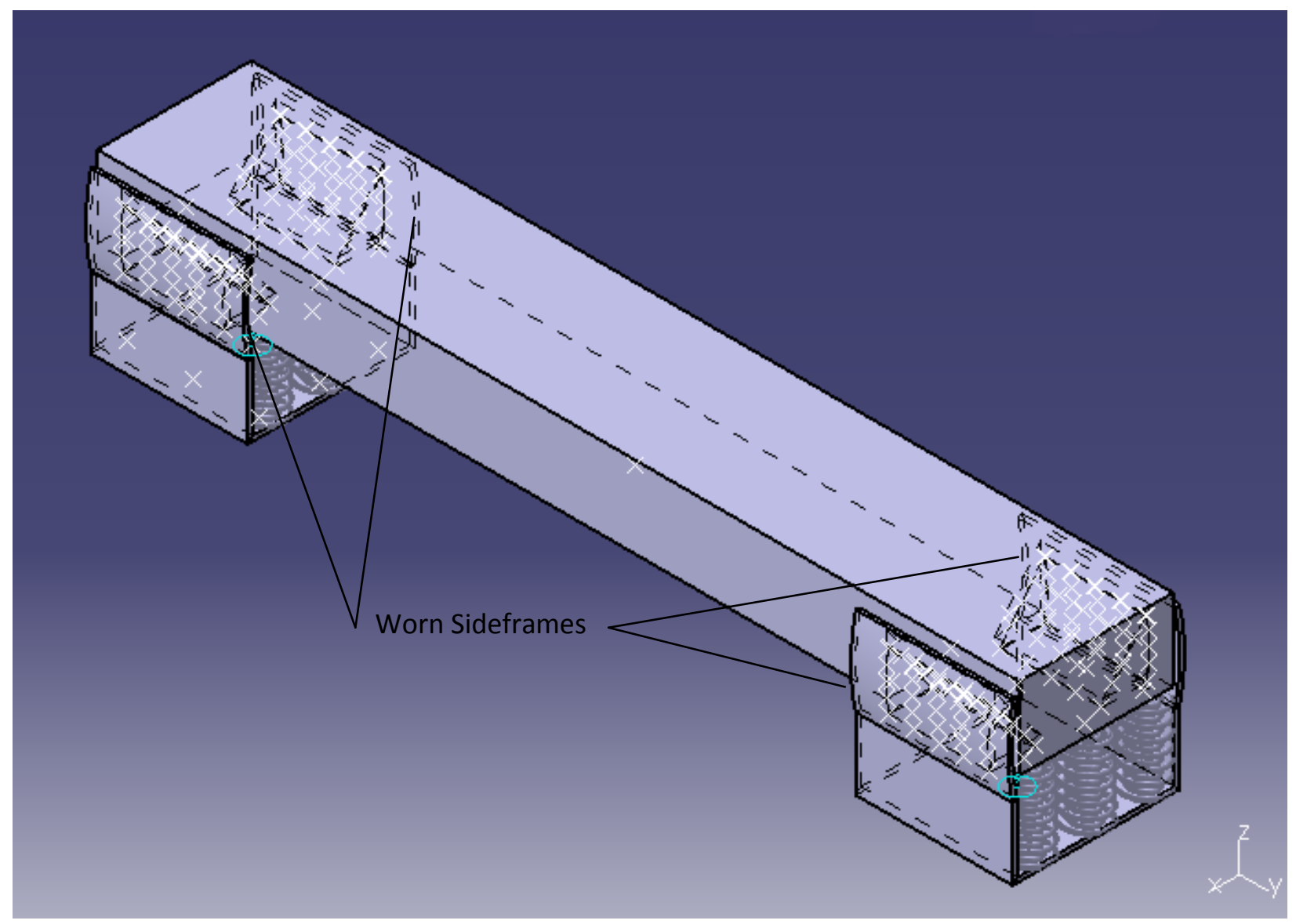

Figure 10. Full-truck model with worn sideframes created in LMS ${ }^{\circledR}$ Virtual.Lab 


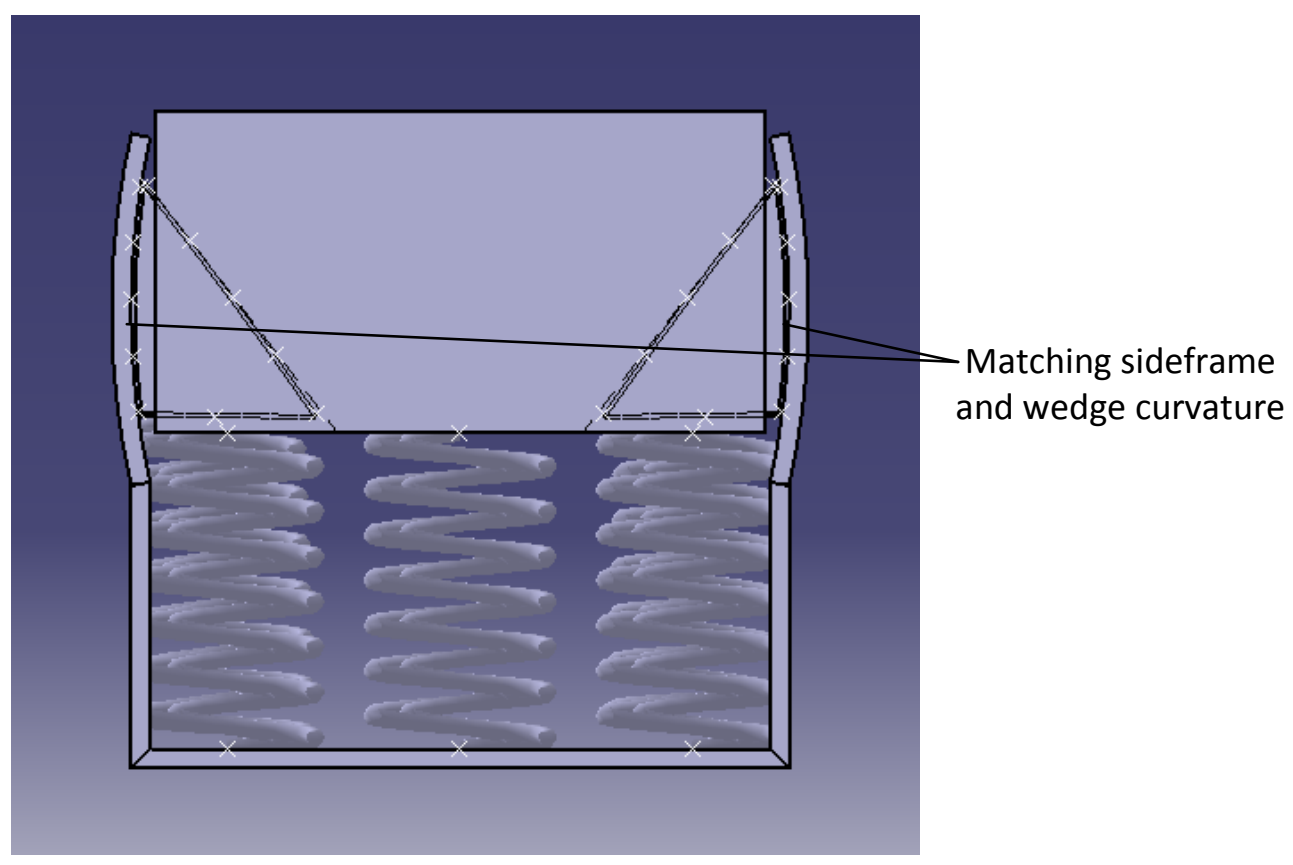

Figure 11. Side view of the worn sideframe model

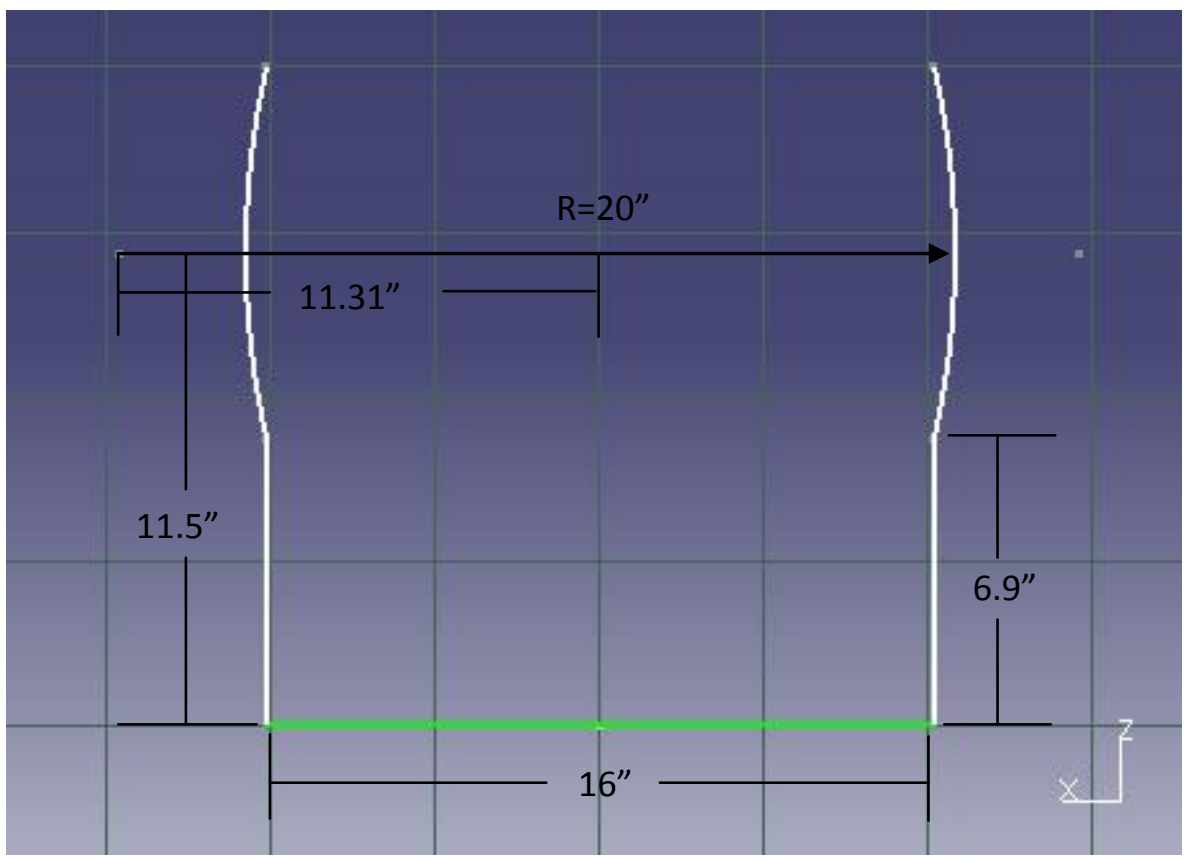

Figure 12. Dimension of sideframe geometry

\subsection{Full-truck Model with Split-Wedges}

The split-wedge is a variation of the friction wedge which uses two wedges on each corner of the bolster. Figure 13 shows a comparison between a standard friction wedge and 
split wedges. As well as having different shapes for the wedges, the bolster contact surface is V-shaped rather than flat, which allows it to contact the faces of each of the split-wedges. The split wedge model was created by modifying the original full-truck model, to incorporate the different wedge and bolster geometries. Figure 14 show the full-truck model with split-wedges.
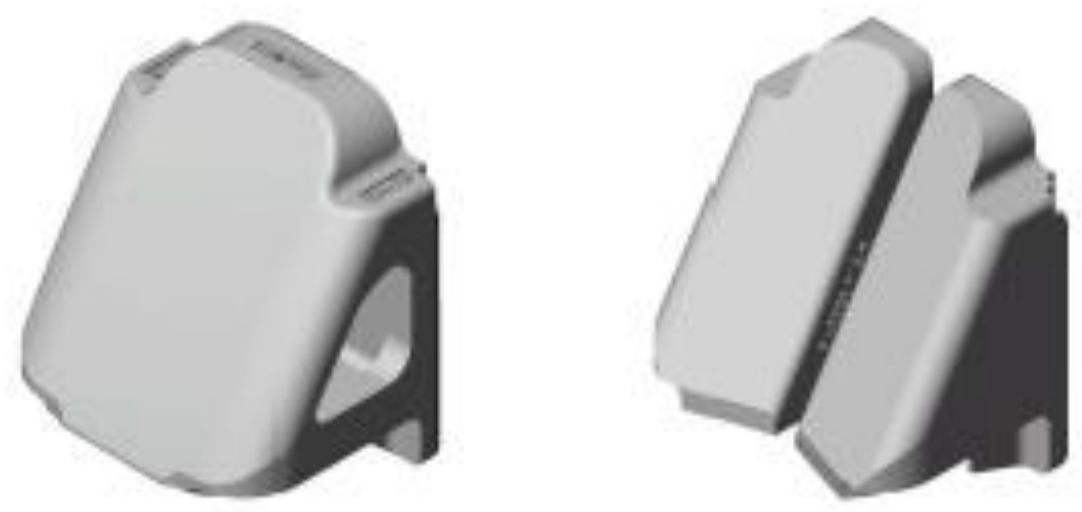

Figure 13. Comparison between a standard friction wedge and a split wedge [10] 


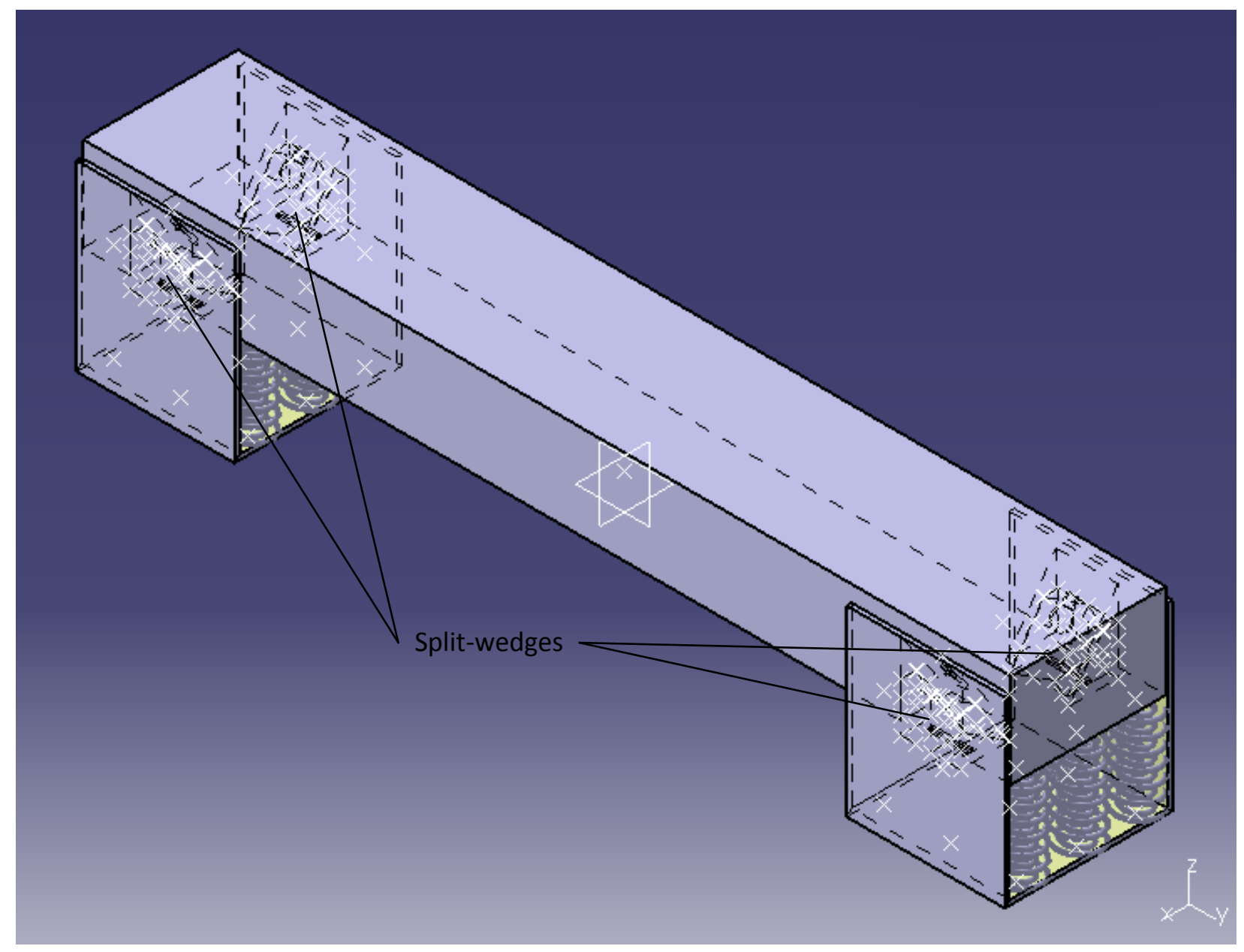

Figure 14. Full-truck model with split wedges

Each of the split-wedges was created as two independent bodies, which are mirror images of each other. As with the other models discussed, the wedges were created by creating a sketch of the wedge shape and extruding it. Due to the more complex angles of the split-wedges, the inner edge of the wedge then was chamfered to create the desired angle of the face. A contact surface was also created on the bolster with matching angles. Figure 15 shows a split-wedge pair that was designed for the $\mathrm{LMS}^{\circledR}$ Virtual.Lab model. The two halves were modeled to allow them to separate from each other as the weight of the bolster was placed on them. Due to the way spring forces are defined in LMS $^{\circledR}$ Virtual.Lab, the halves were not allowed independence in the other directions or rotations in this initial attempt at creating a split-wedge model, in order to keep the complexity of the model down. Figure 16 shows one half of a split-wedge with the parameters labeled. 
Table 5 is a list of the parameter values for the split wedge. We were unable to obtain the inertial properties of the split wedge, so they were made to match those of the standard wedge for the simulations performed in this research.

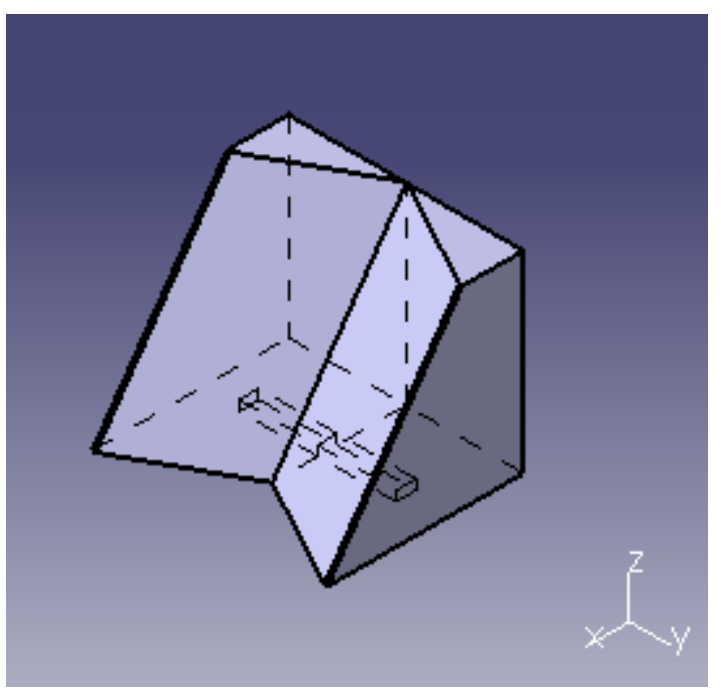

Figure 15. Split-wedge pair as modeled in $\mathrm{LMS}^{\circledR}$ Virtual.Lab

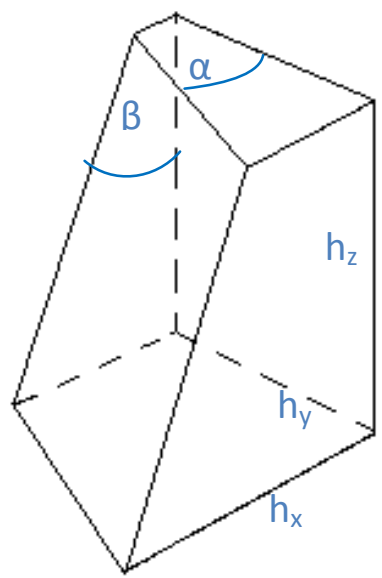

Figure 16. Diagram of half of a split-wedge pair 
Table 5. Wedge properties for the split wedge model

\begin{tabular}{|l|l|}
\hline Wedge $h_{x}($ in) & 5 \\
\hline Wedge $h_{y}($ in) & 3 \\
\hline Wedge $h_{z}$ (in) & 4.88 \\
\hline Wedge $\alpha$ (degrees) & 23 \\
\hline Wedge $\beta$ (degrees) & 35.15 \\
\hline Wedge mass $(\mathrm{lb})$ & 28.15 \\
\hline Wedge $\mathrm{I}_{\mathrm{xx}}\left(\mathrm{lb}\right.$-in $\left.{ }^{2}\right)$ & 10.775 \\
\hline Wedge $\mathrm{I}_{\mathrm{yy}}\left(\mathrm{lb}-\mathrm{in}^{2}\right)$ & 3.803 \\
\hline Wedge $\mathrm{I}_{\mathrm{zz}}\left(\mathrm{lb}-\mathrm{in}^{2}\right)$ & 9.107 \\
\hline
\end{tabular}




\section{Chapter 4. Warp Test and NUCARS ${ }^{\circledR}$ Model}

In this chapter we present the methodologies used to collect benchmark data against which we compared the results of the full-truck model developed. Section 4.1 describes the warp test that was performed at TTCl. Section 4.2 describes the NUCARS ${ }^{\circledR}$ model that was created for a software comparison.

\subsection{Warp Test}

In the spring of 2008, TTCI preformed a series of warp tests at their facility in Pueblo, CO. Receiving data from this test was extremely important, because in the previous work at Virginia Tech, test data was not available for comparison. In Chapter 5 , the data from this test will be used as a benchmark for comparison with the stand-alone LMS Virtual.Lab full-truck model. Warping is a condition where the truck becomes skewed, or one sideframe moves forward while the other moves back with respect to the center of the bolster, as Figure 17 shows.

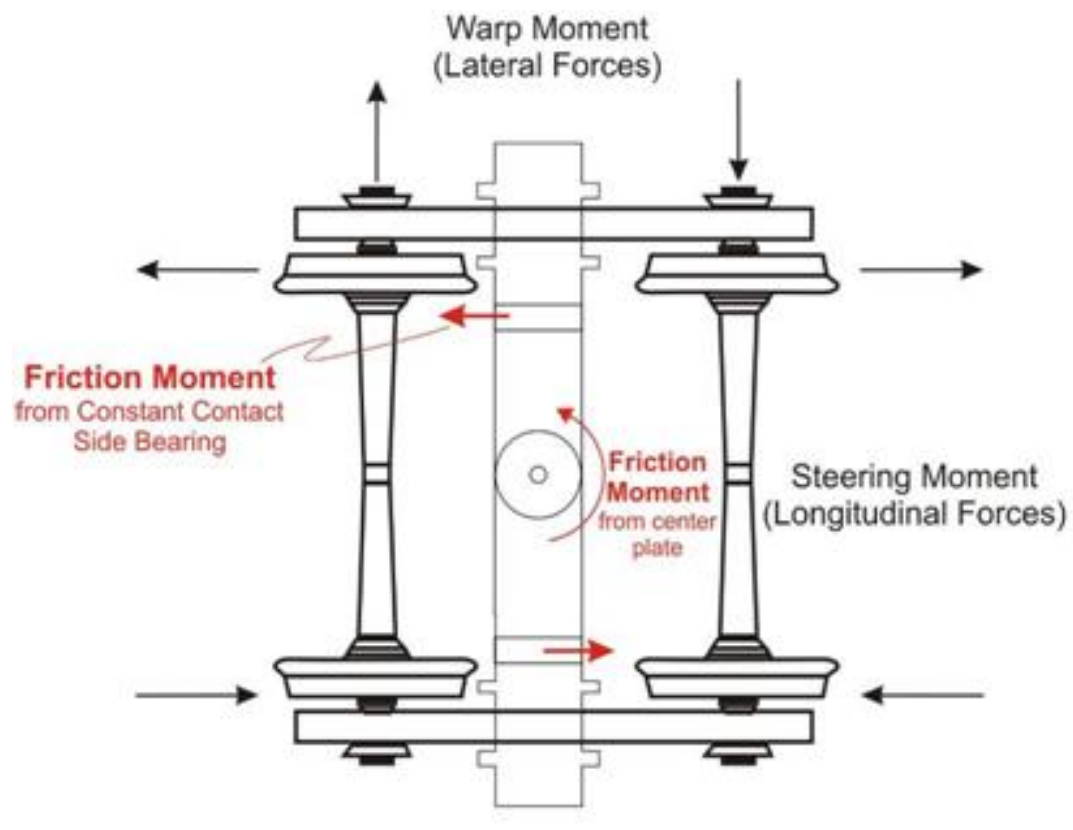

Turning Moment $=$ Warp Moment + Steering Moment

Figure 17. Diagram of truck warp [3] 
The test setup consisted of a car, two actuators, and an instrumented truck. The car was loaded to approximately 120 tons, the weight of a fully loaded hopper. The sideframe was connected to actuators (1), which were used to warp the truck. Figure 18 shows the actuator connection with the sideframe. Another actuator (1) was connected to the car (2) to induce bouncing. Figure 19 shows the actuator connection with the car. These actuators were able to be computer or manually controlled, with either force or displacement inputs. The truck was instrumented to measure the torque resisting the forces placed on the system by the actuators.

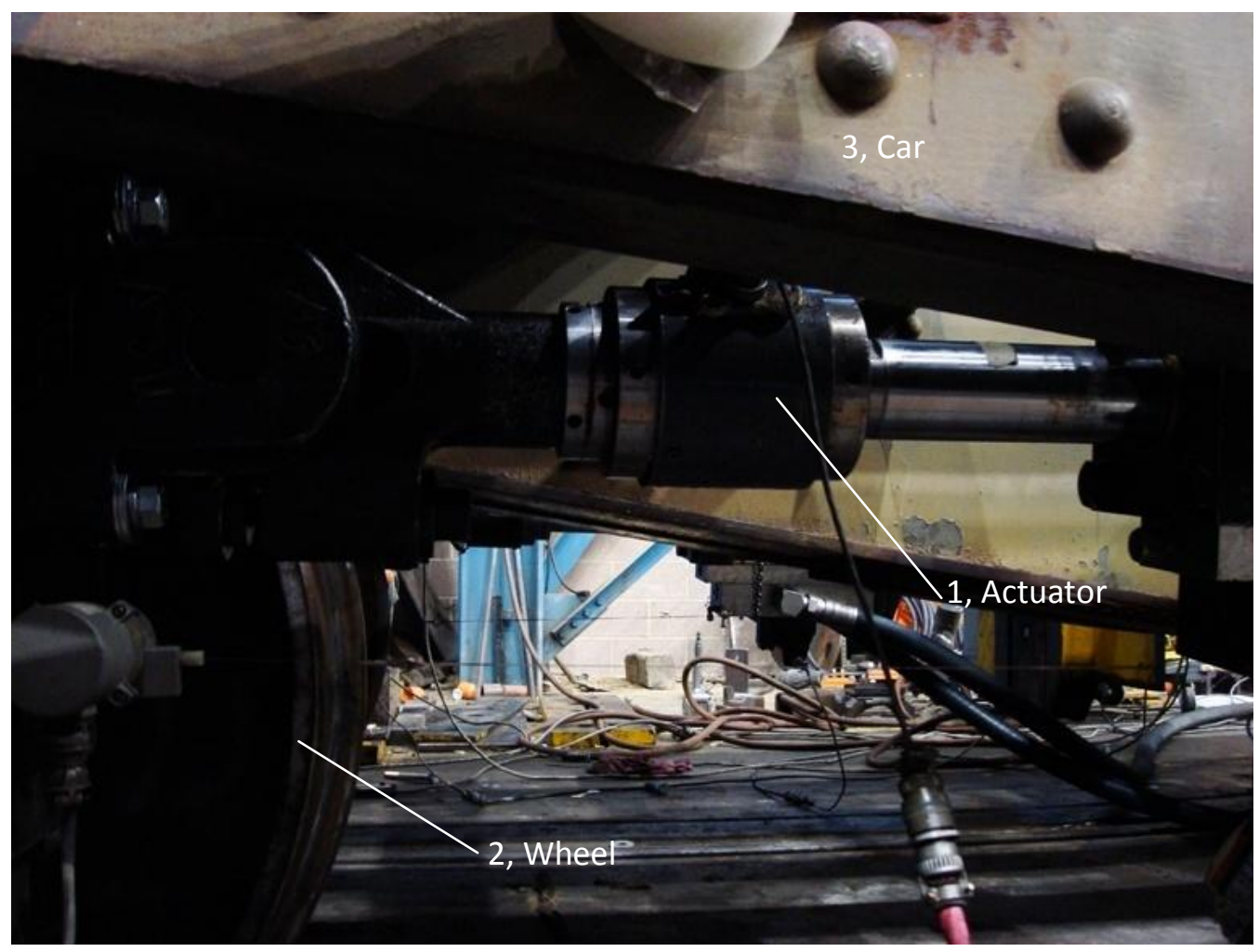

Figure 18. Actuator-sideframe connection 


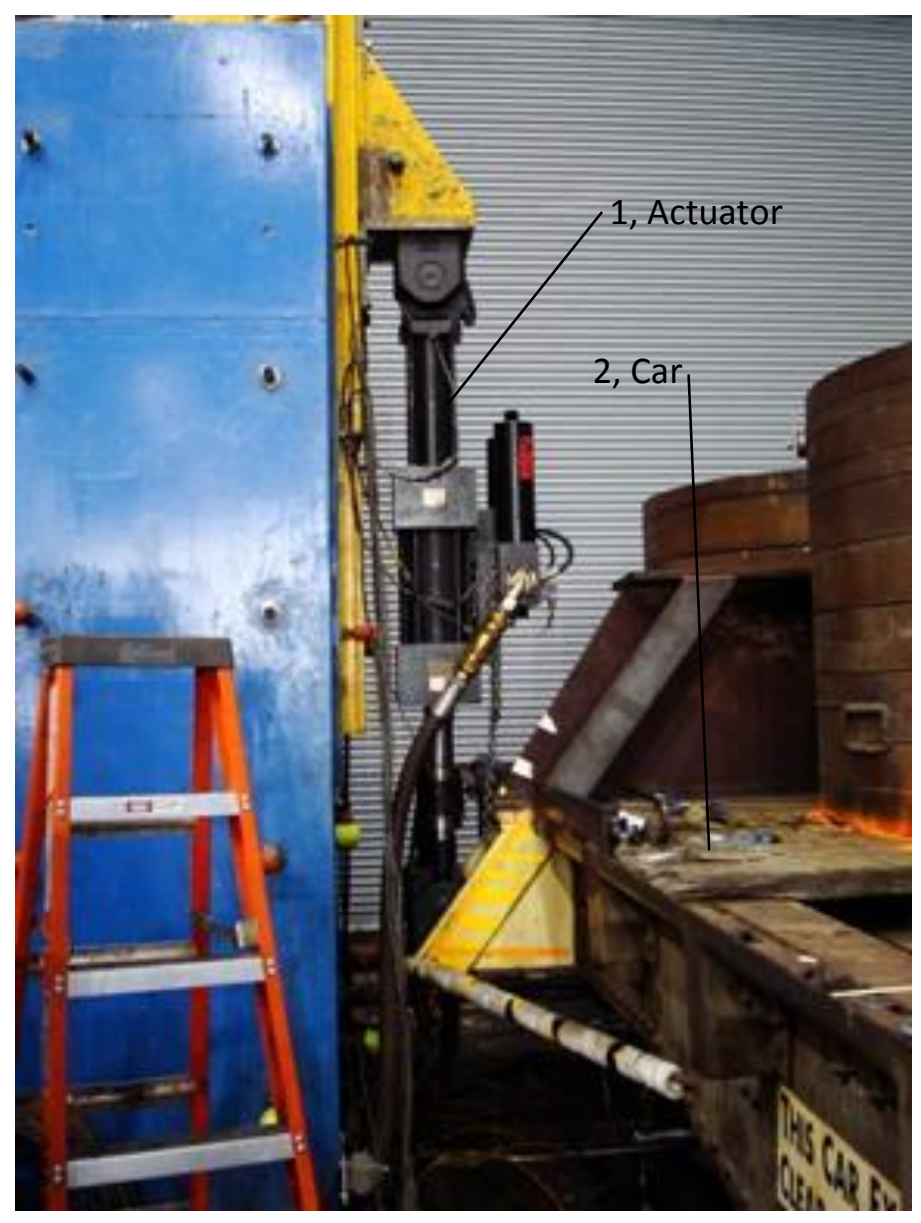

Figure 19. Actuator-car connection

The test that will be used for comparison with the full-truck model was performed using displacement inputs from the actuators to both warp and bounce the truck system. A sinusoidal peak-to-peak bounce of 0.2 inches at $2 \mathrm{~Hz}$ was input through the actuator connected to the car. The actuator controlling the warp was controlled manually in this case. While it was not perfectly sinusoidal, the motion was estimated to have a peak-to-peak amplitude of approximately 2.5 inches at the sideframe, at a frequency of about $0.016 \mathrm{~Hz}$. The results from the test are shown in Figure 20. 


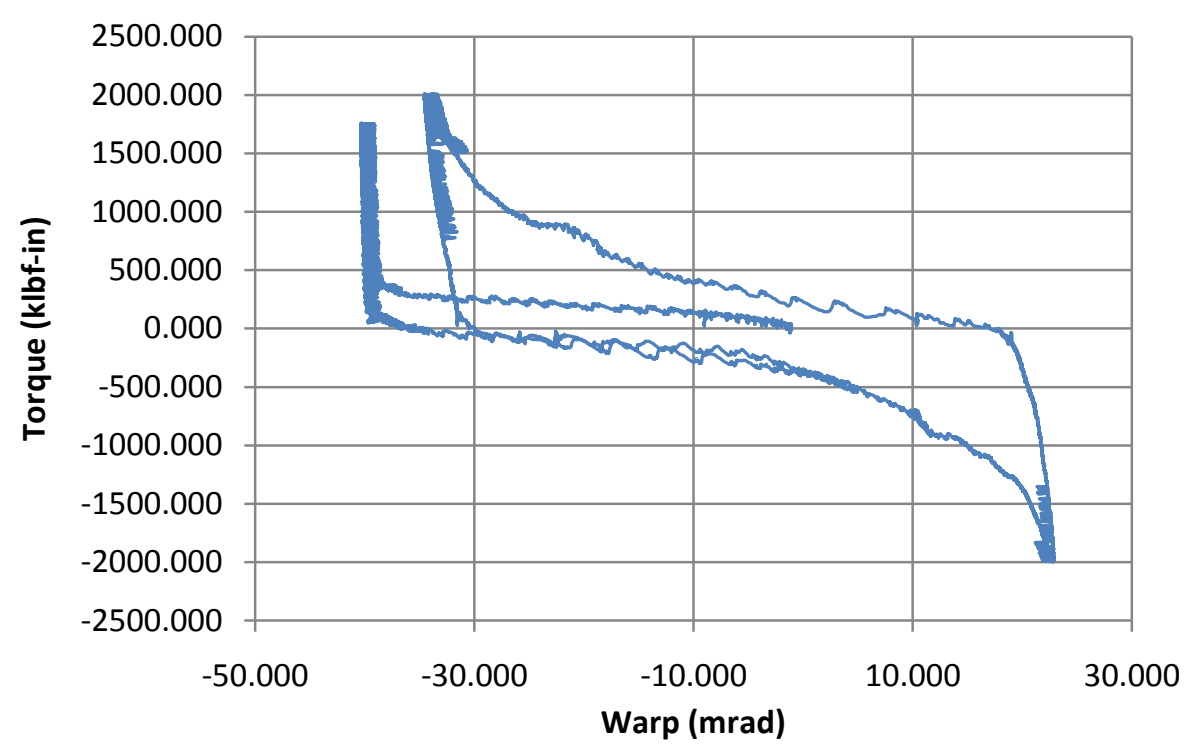

Figure 20. Results from the TTCl warp test

\subsection{NUCARS $^{\circledR}$ Warp Simulations}

NUCARS $^{\circledR}$ is a well establish computer software for multibody dynamics simulations of train systems, developed and maintained by $\mathrm{TTCl}$. NUCARS ${ }^{\circledR}$ simulations were used as a second comparison for the stand-alone full-truck model. Models which match the parameters of the stand-alone model were provided by $\mathrm{TTCl}$, using three different evolutions of wedge element models. The type 6.7 model is a two-dimensional wedge connection, with slider dry friction. This version allows for vertical and lateral displacements of the wedge. The type 6.8 model is also a two-dimensional wedge connection, but includes stick-slip friction capabilities. This version also allows for only vertical and lateral displacements. The type 6.9 model is a three dimensional model with stick-slip friction capabilities. This version allows for vertical, lateral, and longitudinal displacements. All three of these wedge element models neglect mass and inertial properties of the friction wedges.

The simulation cases run in $\mathrm{NUCARS}^{\circledR}$ were configured to match the warp and bounce inputs of the warp test for all three wedge element models. Figure 21 shows that the warp stiffness, or the slope of the torque versus warp curve, is the same for all three NUCARS ${ }^{\circledR}$ wedge elements, with the major difference being how the different models react to the bounce 
input. The model with wedge element 6.7 shows little effect from the bounce input, while the model using element 6.8 shows large increases in torque from the bounce input, and the model using element 6.9 shows a moderate effect. These effects are likely due to the differences in the friction formulations in the three elements.
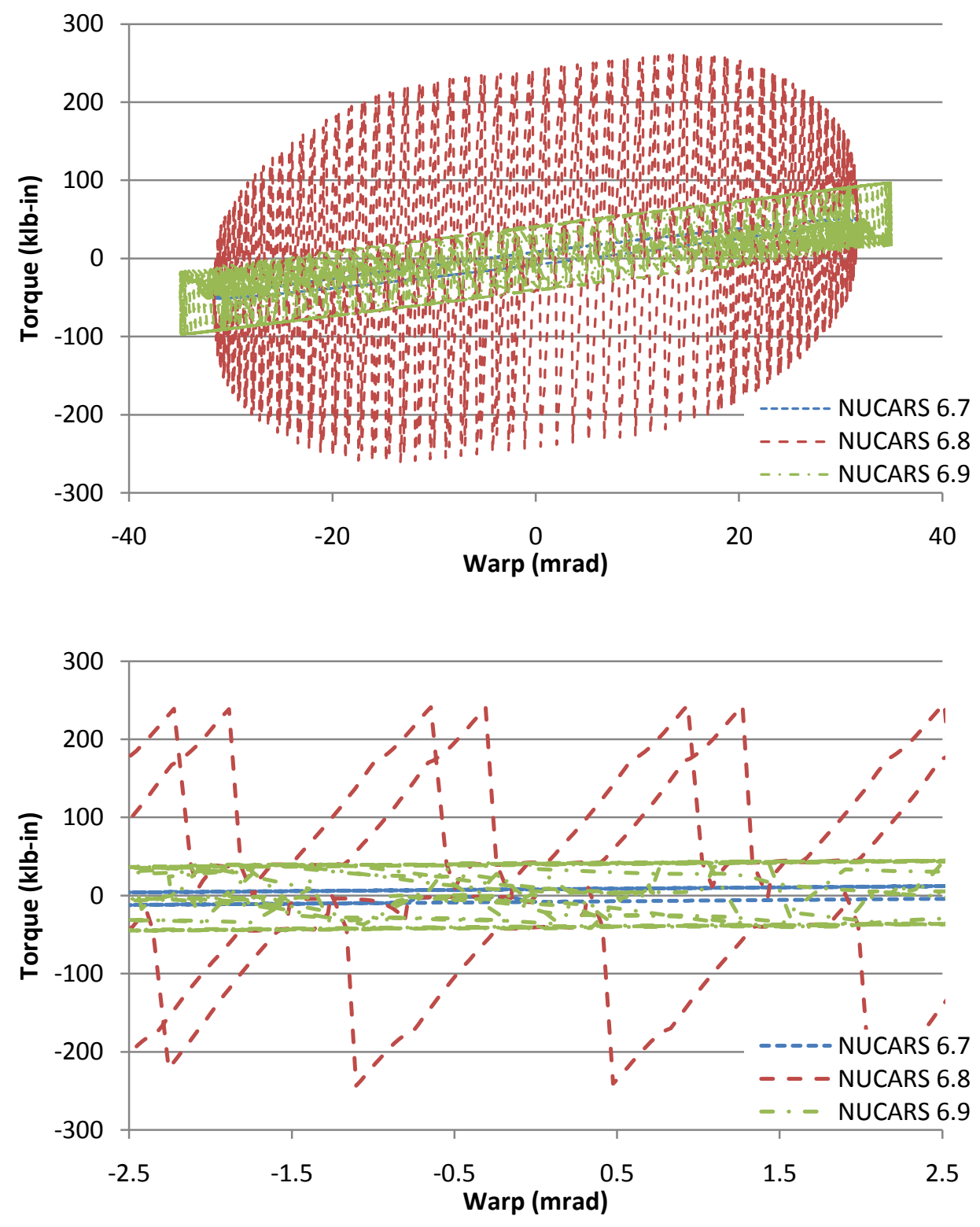

Figure 21. NUCARS ${ }^{\circledR}$ warp stiffness comparison

Figure 22 shows the bolster yaw during the simulation using the three wedge elements. The results show that all three simulations yielded similar results in the general shape of the 
bolster yaw plot, with the exception of the effect of the bounce input. The NUCARS ${ }^{\circledR}$ simulation using wedge element 6.9 showed the bounce input had a slight effect on the bolster yaw, which increased as the warp reached its maximum.

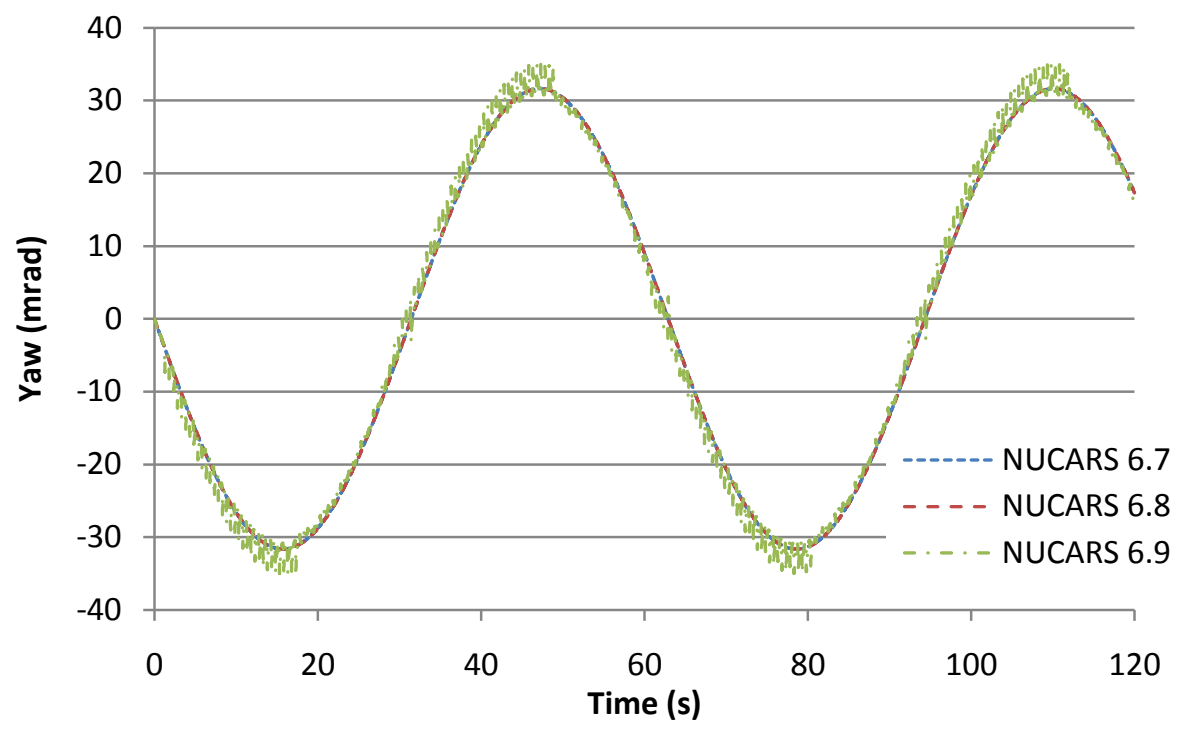

Figure 22. NUCARS ${ }^{\circledR}$ bolster yaw comparison

The forces that the simulation calculated to be on the wedges were also important, as the research is investigating the effects of modeling the wedge as a body. In Figure 23 we see that the vertical force on the wedge is similar for the three wedge element models. The most important aspect that this plot shows is that version 6.9 shows more of a variation due to the warp input, which would be expected in real world operation. In Figure 24 we see that there are significant variations in the lateral force between the three elements. As with the warp stiffness, these differences are likely due to the friction models used in the wedge joint formulations. 

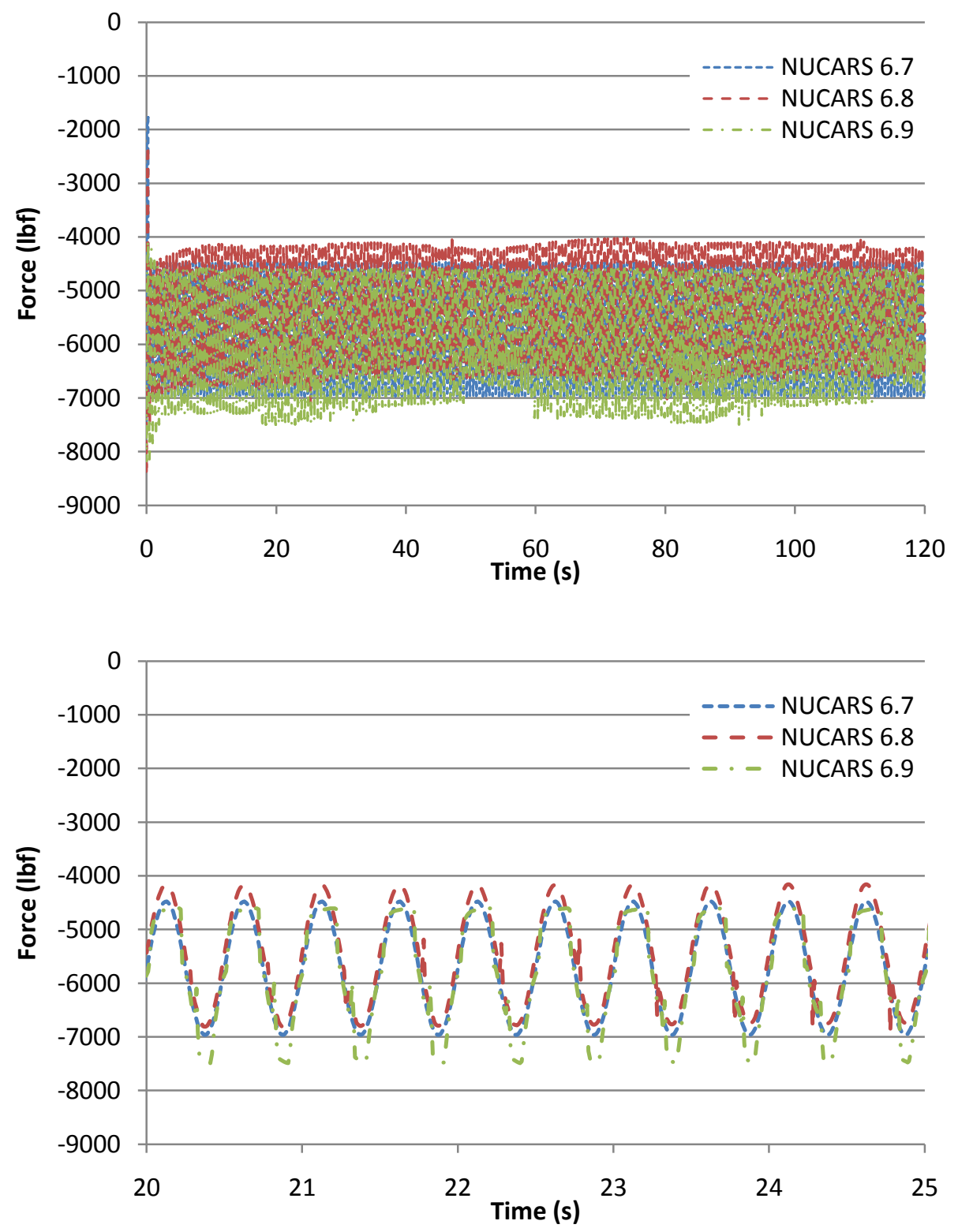

Figure 23. NUCARS ${ }^{\circledR}$ wedge vertical force comparison 

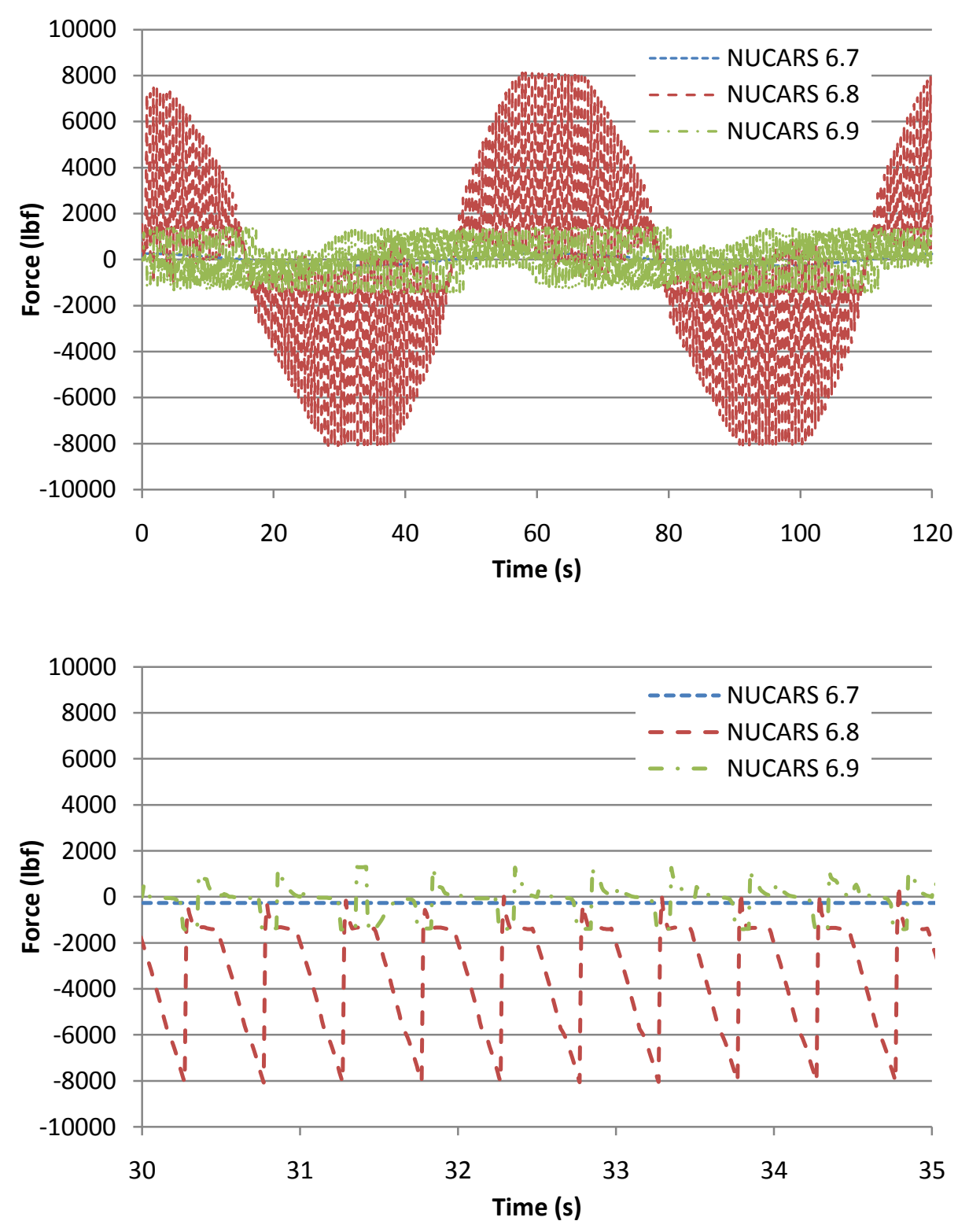

Figure 24. NUCARS ${ }^{\circledR}$ wedge lateral force comparison 


\section{Chapter 5. Results}

This chapter of the thesis will explain the results of the stand-alone models developed in LMS $^{\circledR}$ Virtual.Lab. Section 5.1 focuses on the results of simulations run on the half-truck model. Section 5.2 discusses the results of simulations run on the full-truck model. Section 5.3 discusses the results of the simulations run on the full-truck model with worn sideframes. Section 5.4 discusses the results of the simulations run on the full-truck model with splitwedges.

\subsection{Half-truck Model}

The first simulation in every model and configuration was a dynamic settling run. This consisted of setting the bodies to be in contact, and running a simulation with no external forces or displacements. This allowed the bodies to reach an equilibrium position that would be used as the initial conditions in the simulations with external inputs. Figure 25 is a plot of the bolster and wedge locations during the settling run.

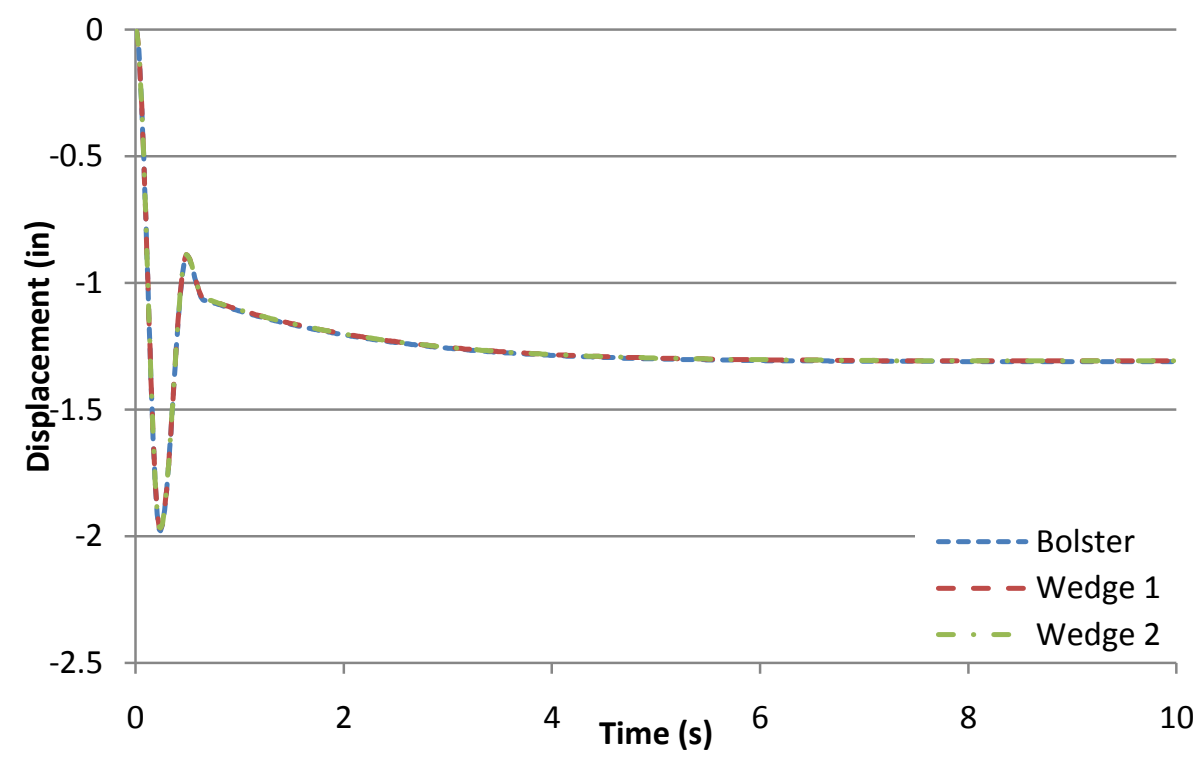

Figure 25. Bodies settling positions for the no-toe geometry case

The half-truck model simulations were conducted with a 0.5 inch displacement of the sideframe at $2.5 \mathrm{~Hz}$. The simulation was repeated for the no-toe, toe-in, and toe-out cases, as 
they were in the $\operatorname{MATLAB}^{\circledR}$ model developed previously $[3,4]$. Figure 26 is a plot of the sideframe displacement input, and the bolster displacement, both relative to ground. In the plot, a lag between the initial movement of the bolster and the steady-state motion is evident. This effect is attributed to the bodies starting at rest, and having to overcome the inertia of the bodies to get them to move. This also causes a background frequency, seen as the amplitude of motion oscillating, which is damped out over time. The plot shows that this background frequency is damped out the fastest in the toe-in configuration, and remains present longest in the toe-out configuration. This is expected behavior, as the toe-in configuration yields more force on the wedge from the sideframe, and thus more damping.

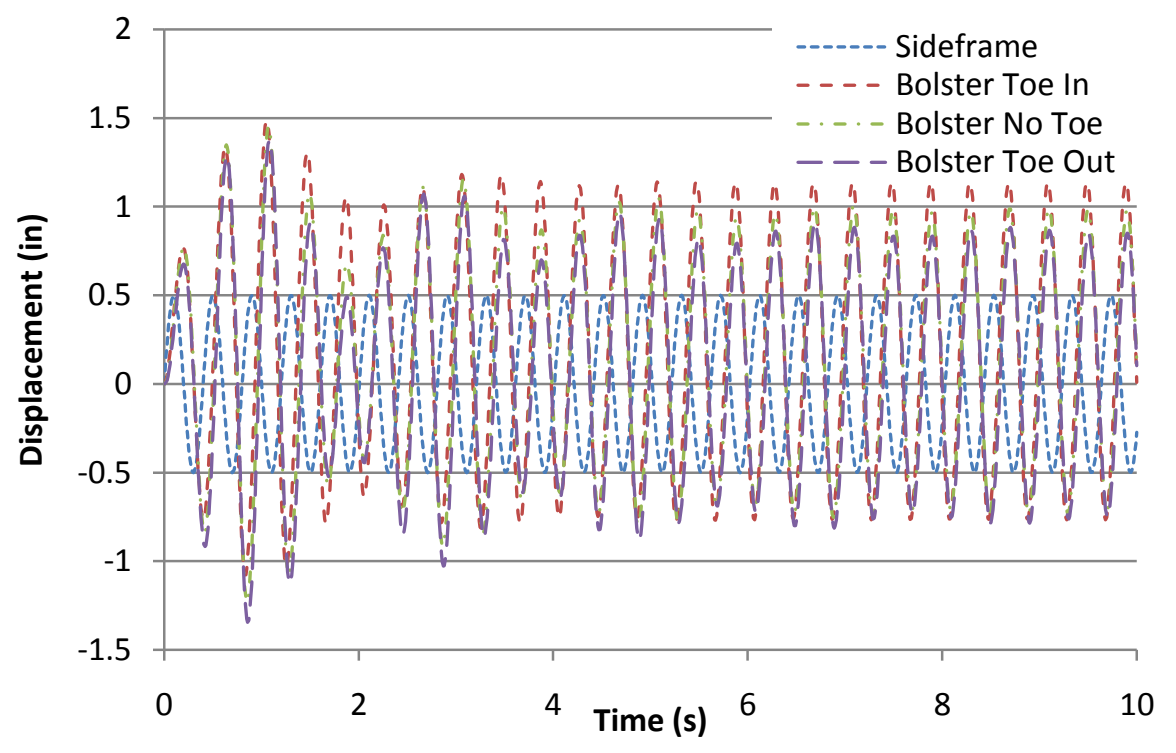

Figure 26. Sideframe and bolster displacements for a 0.5 inch, $2.5 \mathrm{~Hz}$ input

From the same set of simulations, the vertical hysteresis of the three toe cases was examined. Figure 27 is a plot of the vertical hysteresis for the different toe cases. The plot shows that at the bottom of the bounce (left side of plot), the toe-in case experiences the greatest force while the toe-out case experiences the least. This is an expected result, as the sideframes are closer together at the bottom in the toe-in case. This effectively creates a situation where the further down the wedge goes, the more it is pinched between the bolster and sideframe. Conversely, the toe-out case geometry has the sideframes further apart at the bottom, resulting in a looser fit as the wedge moves downward. The plot also shows that the 
motion for the toe-in case centers on a higher location than the no-toe and toe-out cases. Again, this is expected for the reasons listed above.

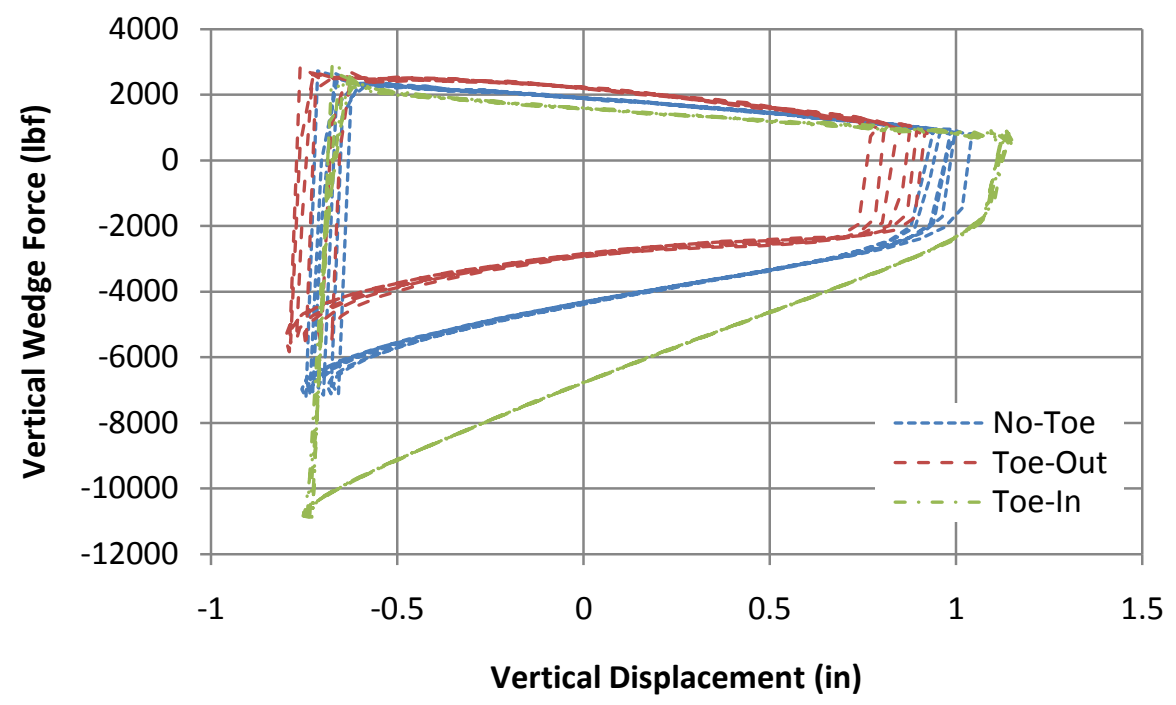

Figure 27. Vertical wedge force hysteresis loops for all toe cases

In order to maintain continuity with the previous work done at Virginia Tech, a comparison between parameters of the model had to be performed. Figure 28 is a plot of the vertical force on one of the wedges using the 0.5 inch, $2.5 \mathrm{~Hz}$ sideframe input. Figure 29 is a plot of the vertical force on one of the wedges under similar conditions in the MATLAB ${ }^{\circledR}$ model. Although the frequency and amplitude of these two plots were different, resulting in different values for the amplitude of the forces, the shape of the force plots is very similar, which confirms that the $\mathrm{LMS}^{\circledR}$ Virtual.Lab model captures the behavior of friction wedges similarly to the MATLAB ${ }^{\circledR}$ model. 


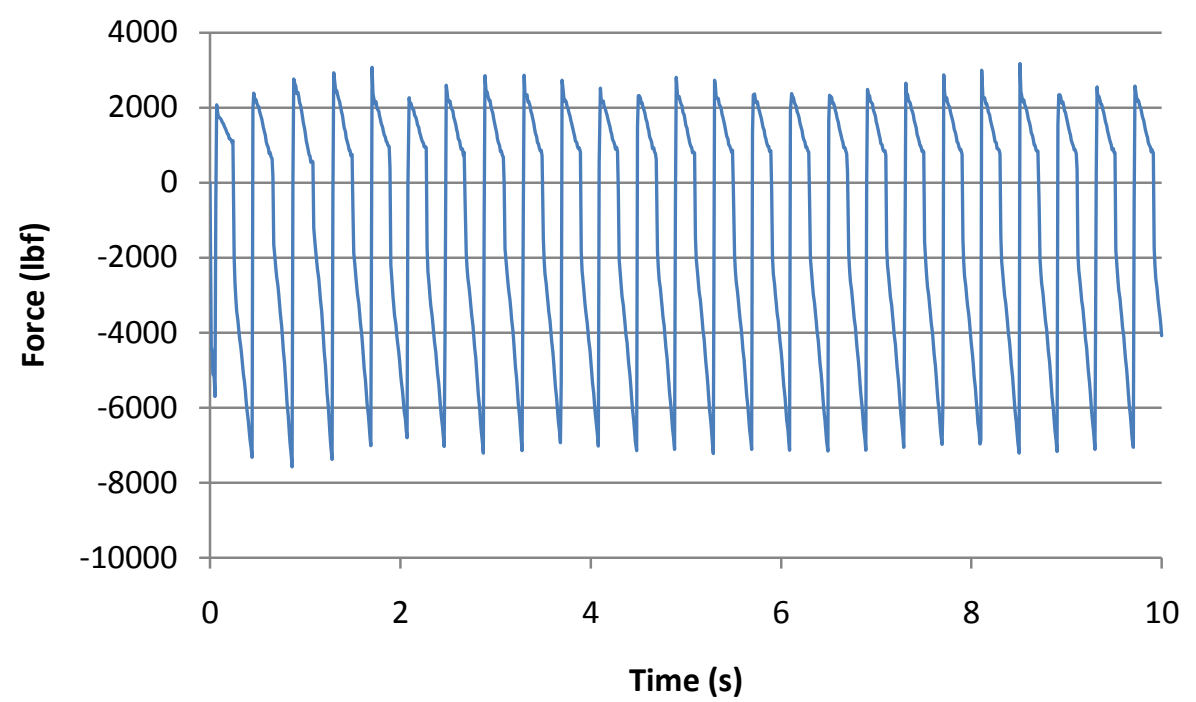

Figure 28. Vertical forces on the wedge from the $\mathrm{LMS}^{\circledR}$ Virtual.Lab model

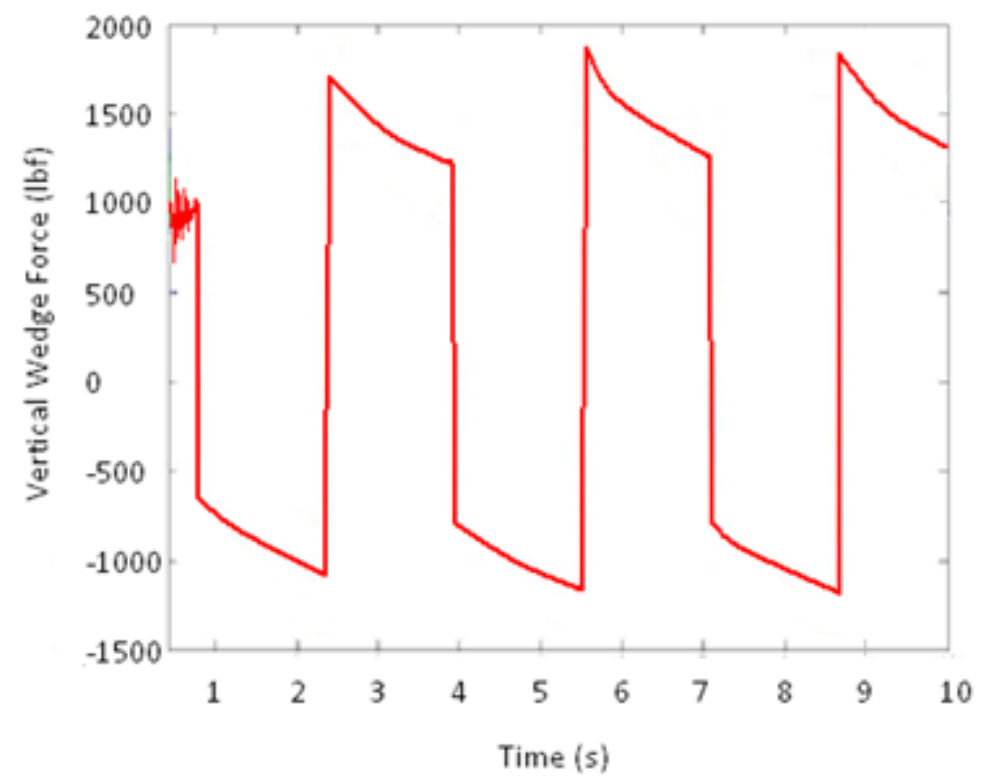

Figure 29. Vertical forces on the wedge from the MATLAB ${ }^{\circledR}$ model 


\subsection{Full-truck Model}

The first simulation run on the full-truck model was the dynamic settling run, to find the initial conditions to be used for further simulations. Because the full-truck model is not simply the half-truck model mirrored, but a model developed from scratch, having minor geometry differences with the half-truck model, it was not safe to just assume the same settling positions. Figure 30 is a plot of the settling positions of the bolster and of the friction wedges.

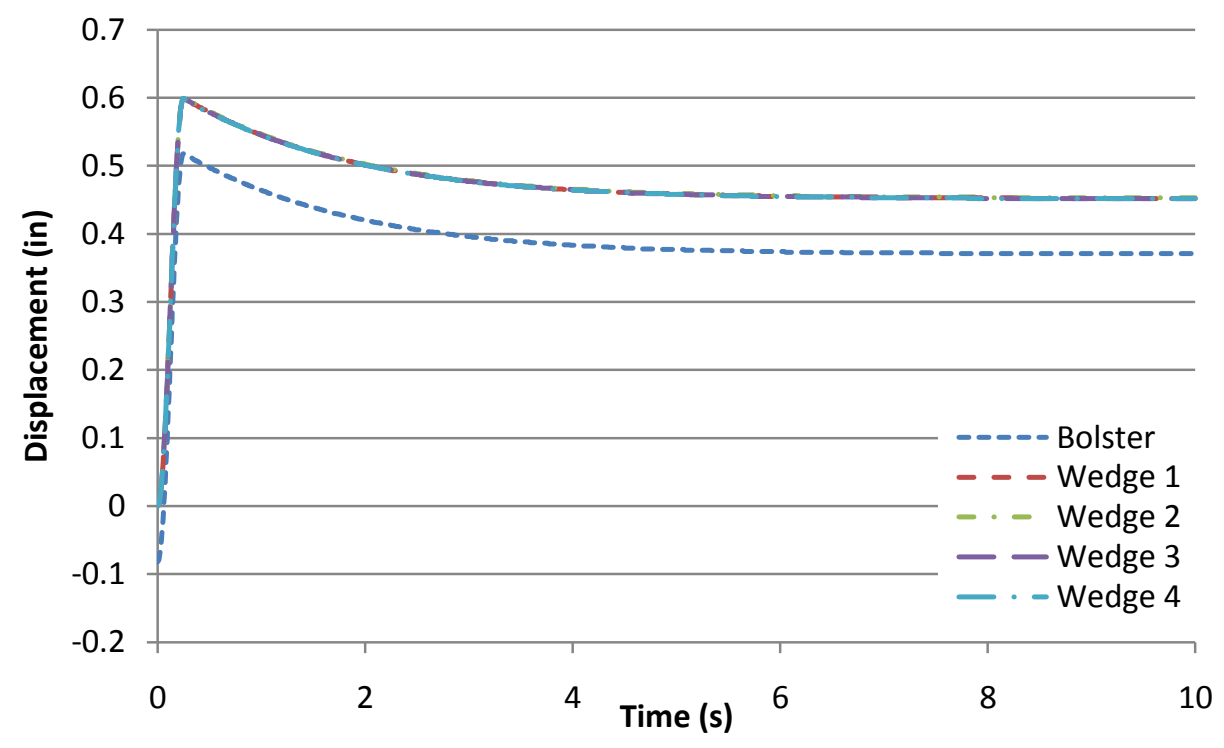

Figure 30. Bodies settling positions for the full-truck model

To ensure that the full-truck model has a realistic behavior, it was first compared with the half-truck model. Figure 31 is a plot of the vertical forces on one of the wedges on the fulltruck and the half-truck, using the 0.5 inch, $2.5 \mathrm{~Hz}$ bounce input. The plot shows that the fulltruck model performed similarly to the half-truck model. With the confidence that the fulltruck model was performing as expected, further simulations were run. 


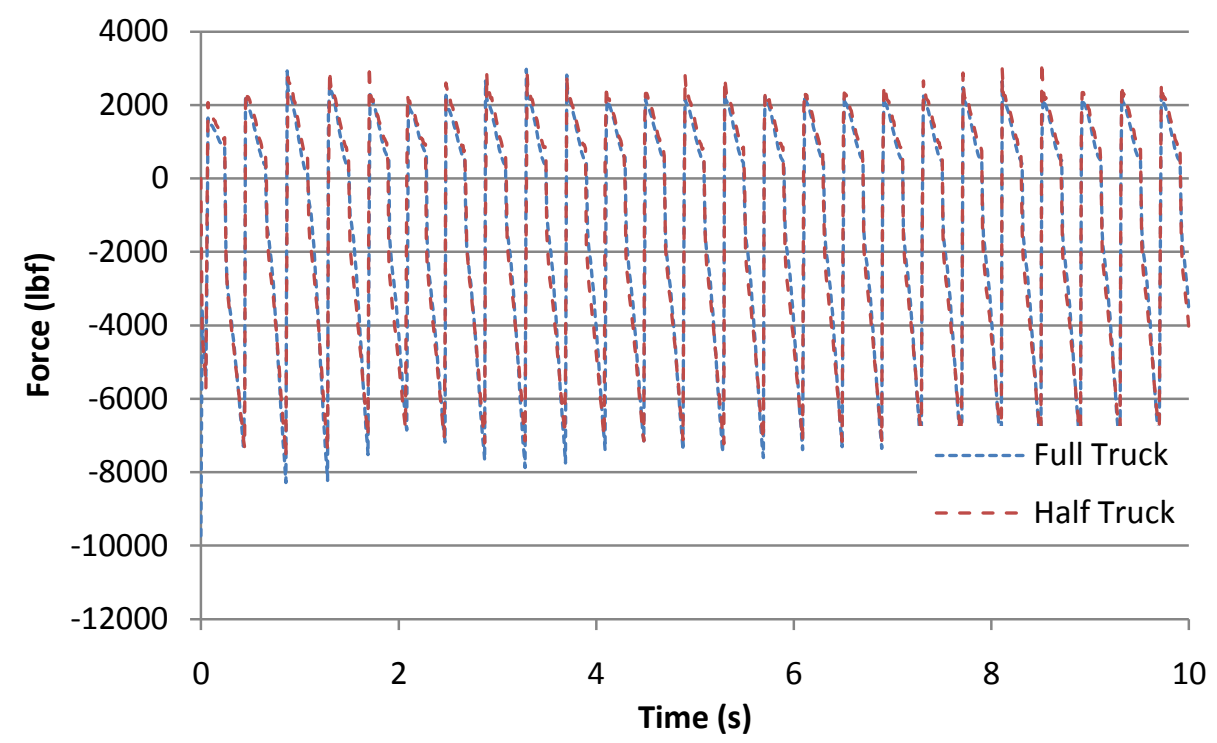

Figure 31. Vertical wedge force comparison between the full-truck and the half-truck models

After making minor geometry changes to the wedges, to better match the system used by $\mathrm{TTCl}$ in the warp test, the 0.5 inch, $2.5 \mathrm{~Hz}$ bounce simulation was run again. With this set of simulation parameters the simulation was run on all three full-truck models discussed in this thesis. In later sections, the worn sideframe and split-wedge models will be analyzed using the following results from the full-truck model as a benchmark. Figure 32 is a plot of the vertical displacements of selected bodies of the system. In the plot we can see that the bolster and wedge have similar displacements, because they were in contact at all times during this simulation. Figure 33 is a plot of the pitch angle of the friction wedges, which show that the flat surface contact between the wedges and the sideframes allow for little pith. Figure 34 is a plot of the vertical force on one of the wedges. As mentioned in the comparison with the half-truck model, the shape of the vertical force curve compares well with data from previous models. Figure 35 is a plot of the longitudinal forces on the same wedge. The longitudinal force also compares well with data from previous models. 


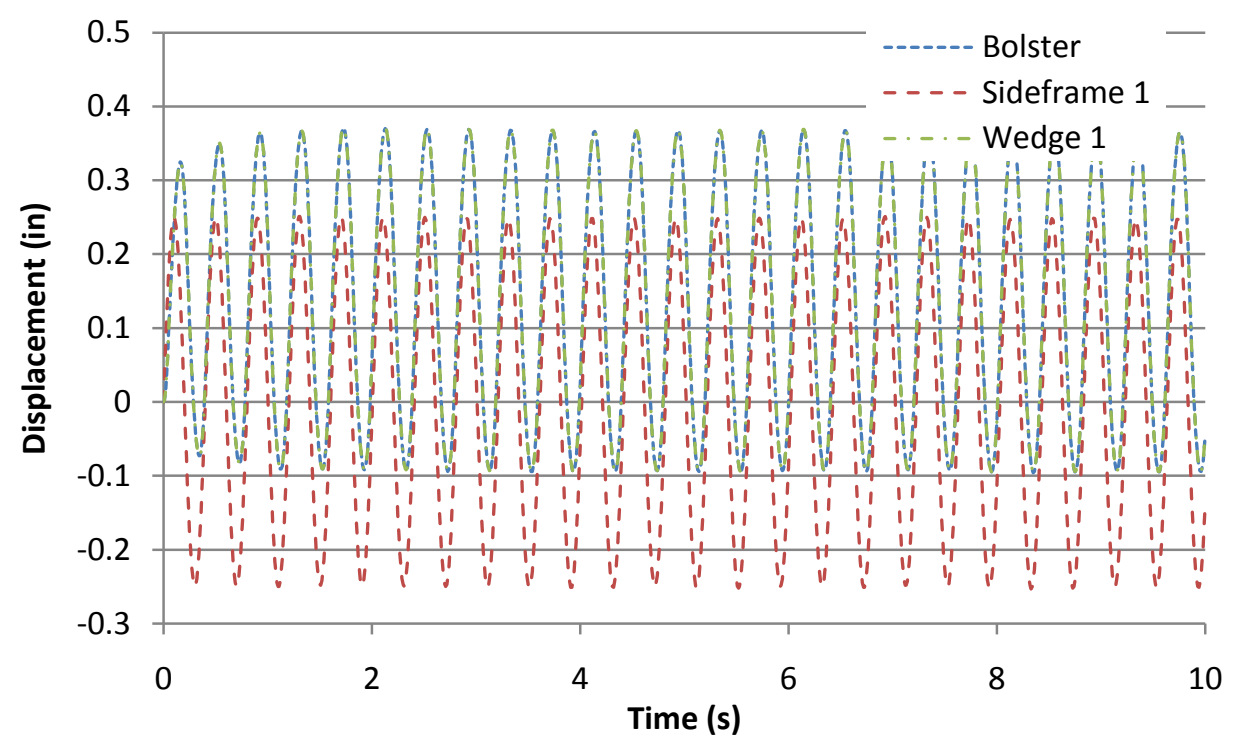

Figure 32. Vertical displacement during bounce simulation

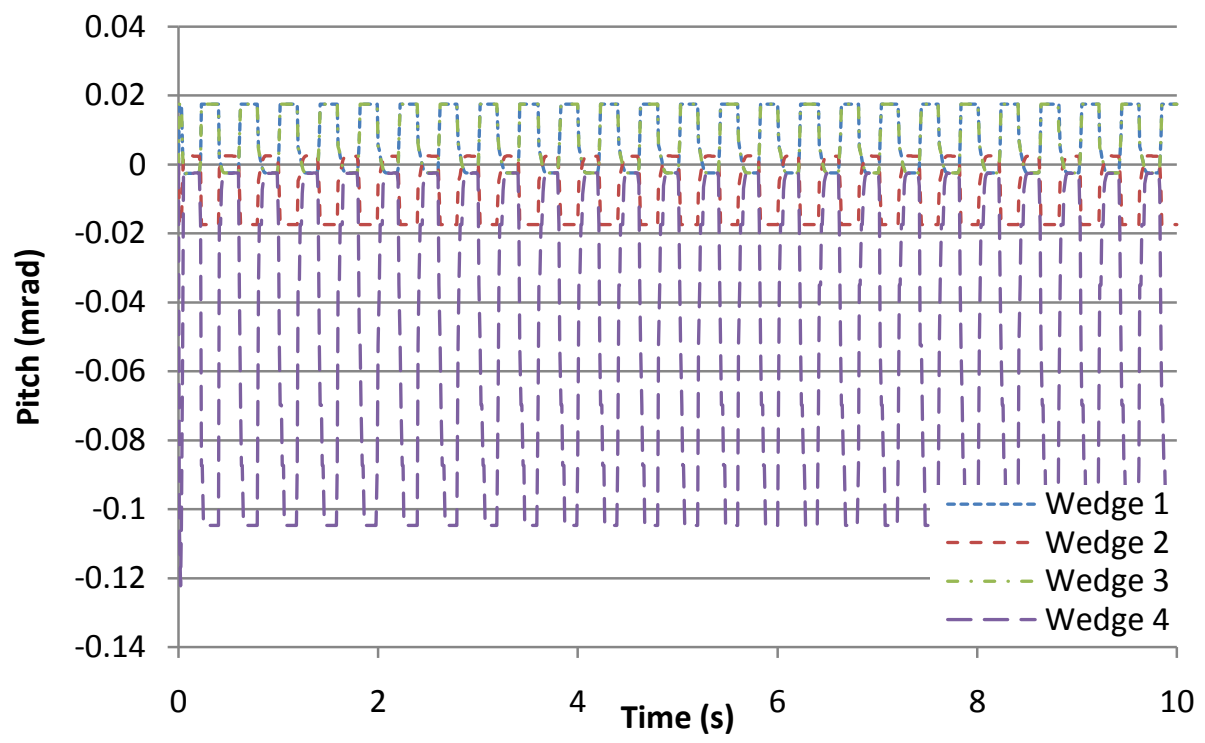

Figure 33. Pitch angle of the friction wedges during bounce simulation 


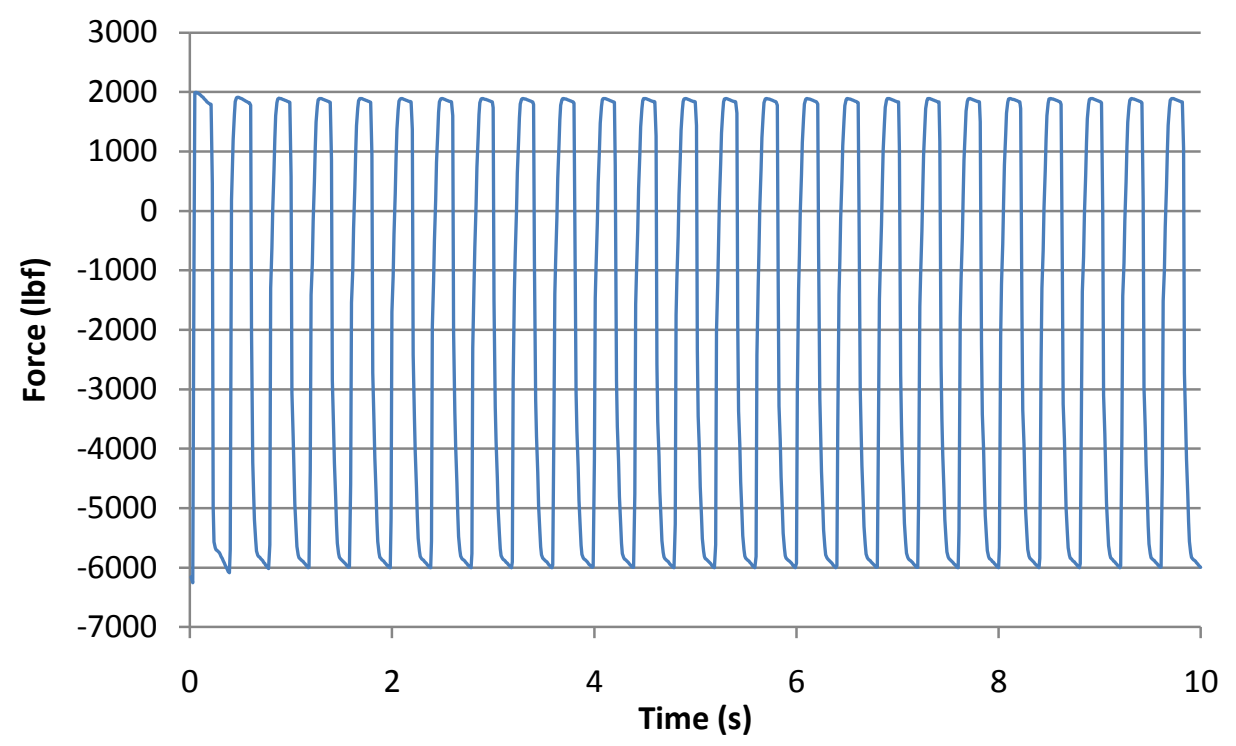

Figure 34 . Vertical force between wedge 1 and the sideframe

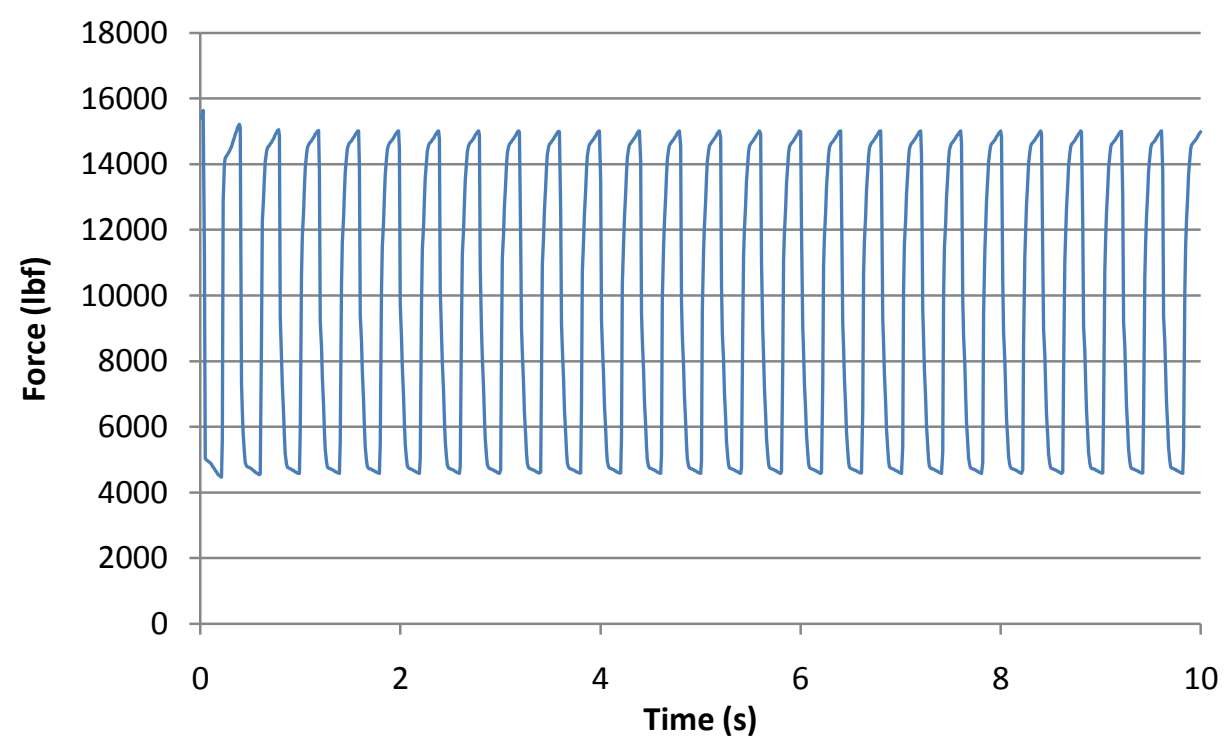

Figure 35. Longitudinal force between wedge 1 and the sideframe

The validation of the stand-alone model against the test data collected at TTCI was one of the major goals of this research. The displacement inputs to the model were made to closely match those of the test, with a 0.2 inch amplitude bounce at $2 \mathrm{~Hz}$, and a 2.5 inch amplitude sideframe displacement at $0.016 \mathrm{~Hz}$. Figure 36 is an overlay plot of the torque on the bolster of the stand-alone model and the test data. The test data was shifted to center the warp around zero, as the instruments were not zeroed before the test. This allowed for a better comparison 
with the stand-alone model. The main focus was for the model to be able to accurately output the warp stiffness, or the slope of the torque versus warp plot. The plot shows a good correlation between the test and the model until the extremes, where the test data had rapid increases in torque. When taking the average of the center $40 \mathrm{mrad}$ of motion, we found a warp stiffness of about $20 \mathrm{klbf}-\mathrm{in} / \mathrm{mrad}$ for both the model and the test. It is important to note that the model does not simulate the entire sideframe structure, or the axles and the wheel/rail contact, while the test was done on a fully operational truck system. We believe that these other components of the truck may add stiffness to the system, and could be the reason for the sharp peaks. Another factor that we believe contributes to the sharp peaks in the test data is that as the wedges slow down, they reach the threshold where the stick phase of stick-slip friction takes over. When the wedges begin sticking, the entire system becomes more rigid, which increases the warp stiffness.

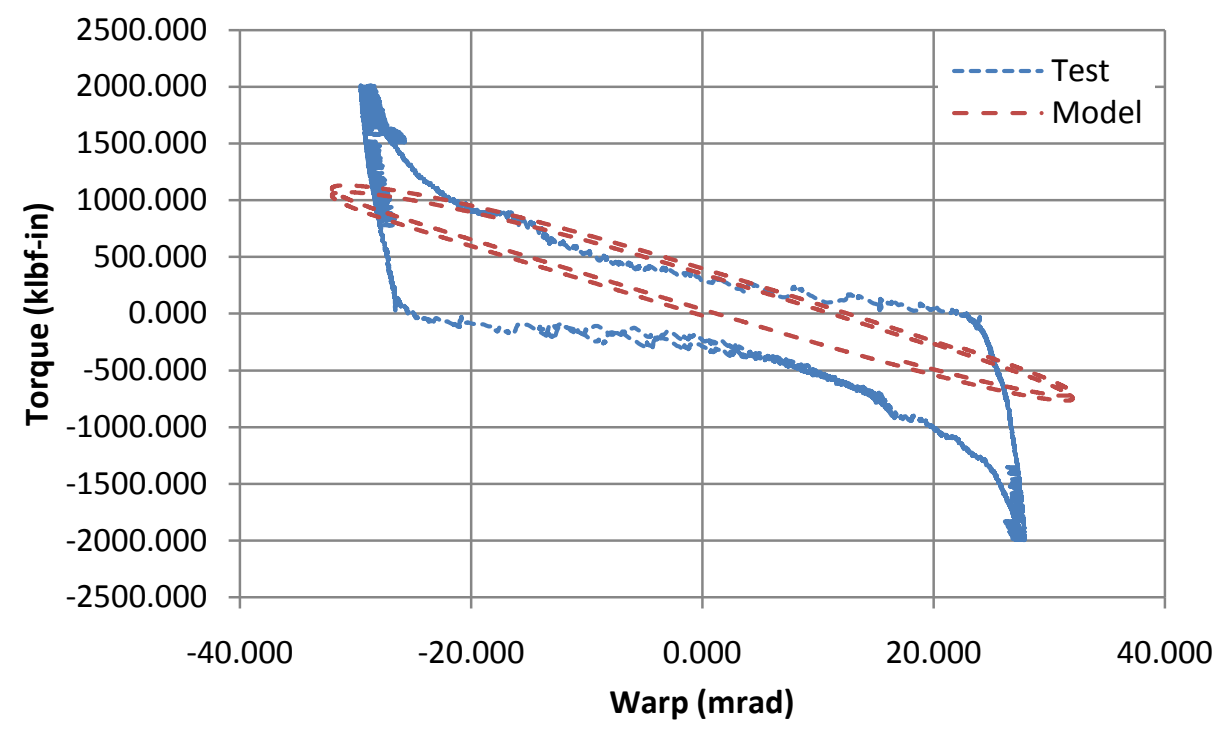

Figure 36. Warp stiffness comparison between $\mathrm{LMS}^{\circledR}$ Virtual.Lab model and test data

The stand-alone model's results were also compared to the results of the NUCARS ${ }^{\circledast}$ simulations that were run to match the inputs of the test. As discussed in the previous chapter, three different versions of the wedge element in NUCARS ${ }^{\circledR}$ were used for comparison purposes. By comparing these results, the goal was to determine if modeling the wedges as bodies enables the stand-alone model to capture the behavior of the truck better under 
warping, which has been thought to be a weakness of the current approach to modeling wedges in NUCARS ${ }^{\circledR}$. Figure 37 is a plot of the calculated torque on the bolster from the standalone model and from NUCARS ${ }^{\circledR}$. Based on the results from the test, and the correlation between the test and the stand-alone model, the plot reveals that the warp stiffness output from all of the NUCARS ${ }^{\circledR}$ models is significantly lower than expected.
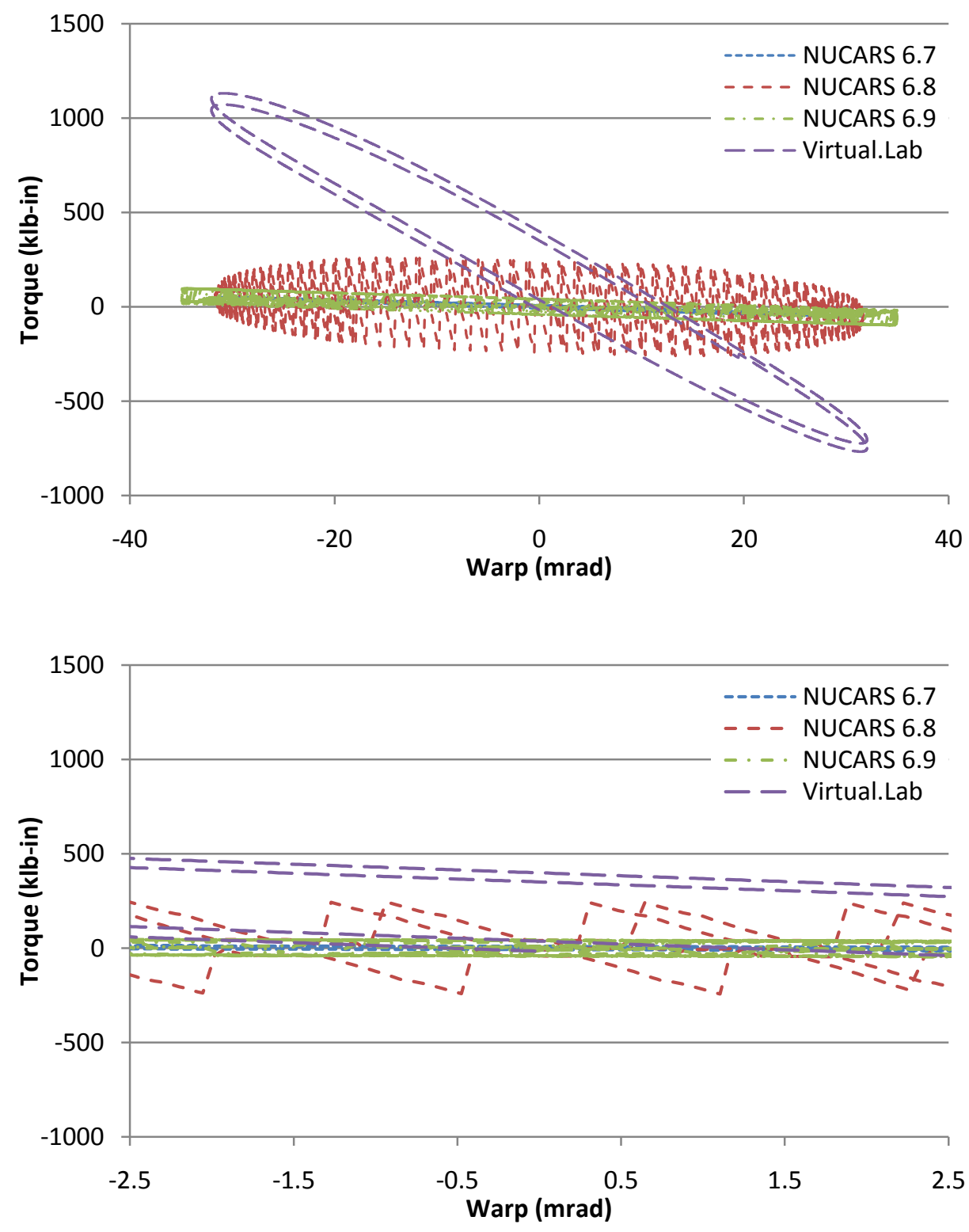

Figure 37. Warp stiffness comparison between LMS $^{\circledast}$ Virtual.Lab model and NUCARS ${ }^{\circledast}$

To further investigate the effects of modeling the wedges as bodies with geometry rather than connection joint equations, other important output parameters from the stand- 
alone model were compared with the NUCARS ${ }^{\circledast}$ outputs. Figure 38 is a plot of the yaw angle of the bolster over the time period of the run for both the stand-alone model and for NUCARS ${ }^{\circledR}$. A significant difference in bolster yaw is seen in the stand-alone model, which is largely due to the wedge geometry. The simulation showed that there was a significant rise of the trailing wedges (relative to their particular sideframe's motion) as the sinusoidal warp began to slow before changing direction, at around 15 seconds. The geometry of the wedge then kept the bolsters yaw slightly skewed for the remainder of the simulation. This case is a prime example of the shortcomings of modeling a wedge as connection with no geometry rather than as a solid body.

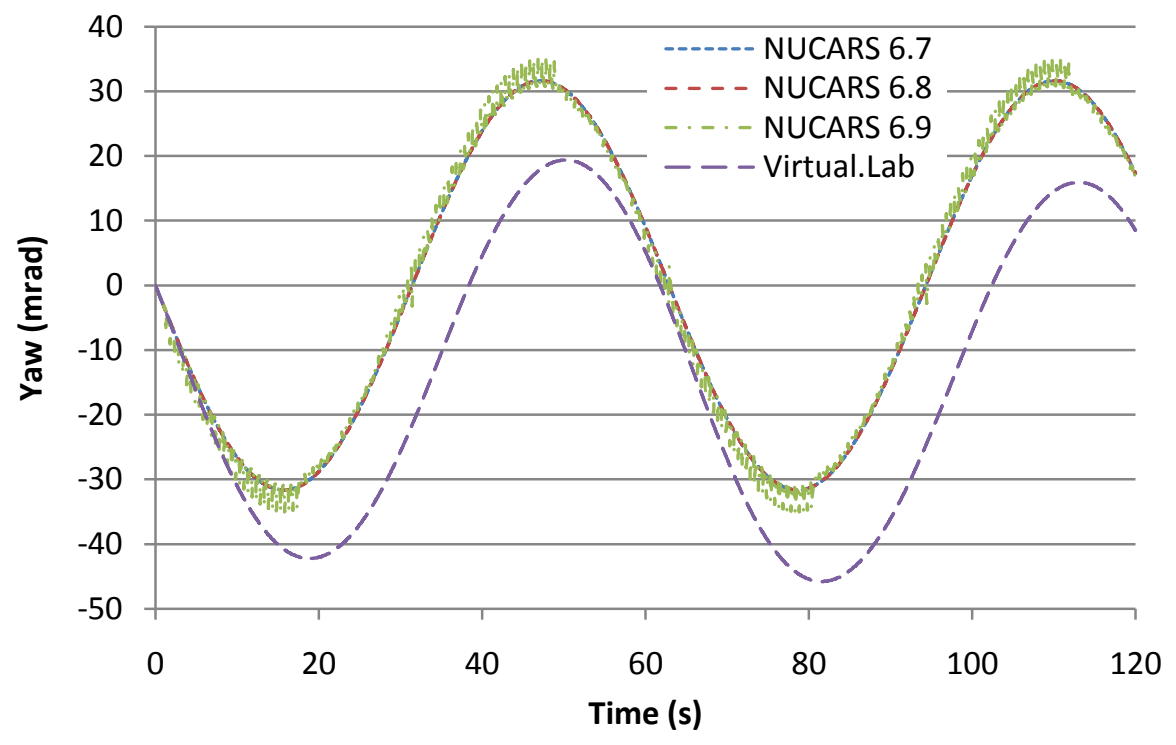

Figure 38. Bolster yaw comparison between LMS ${ }^{\circledast}$ Virtual.Lab model and NUCARS ${ }^{\circledast}$

The vertical force on the friction wedges is an important parameter to investigate, as it is the force that damps the movement of the system. NUCARS ${ }^{\circledR}$ defines the vertical force on the wedge as a combination of the force from the spring and the vertical friction force, so for any comparisons with NUCARS ${ }^{\circledR}$, the vertical force outputs from the stand alone model were made to do the same. The sign convention was also switched to match that of NUCARS ${ }^{\circledR}$. Figure 39 shows that in all of the NUCARS ${ }^{\circledR}$ simulations, the vertical force stays relatively steady throughout the warping simulations. The stand-alone model, however, indicated significant decreases in the vertical force at times throughout the run. It was found that these decreases in vertical force correspond with the wedge rise mentioned before. 

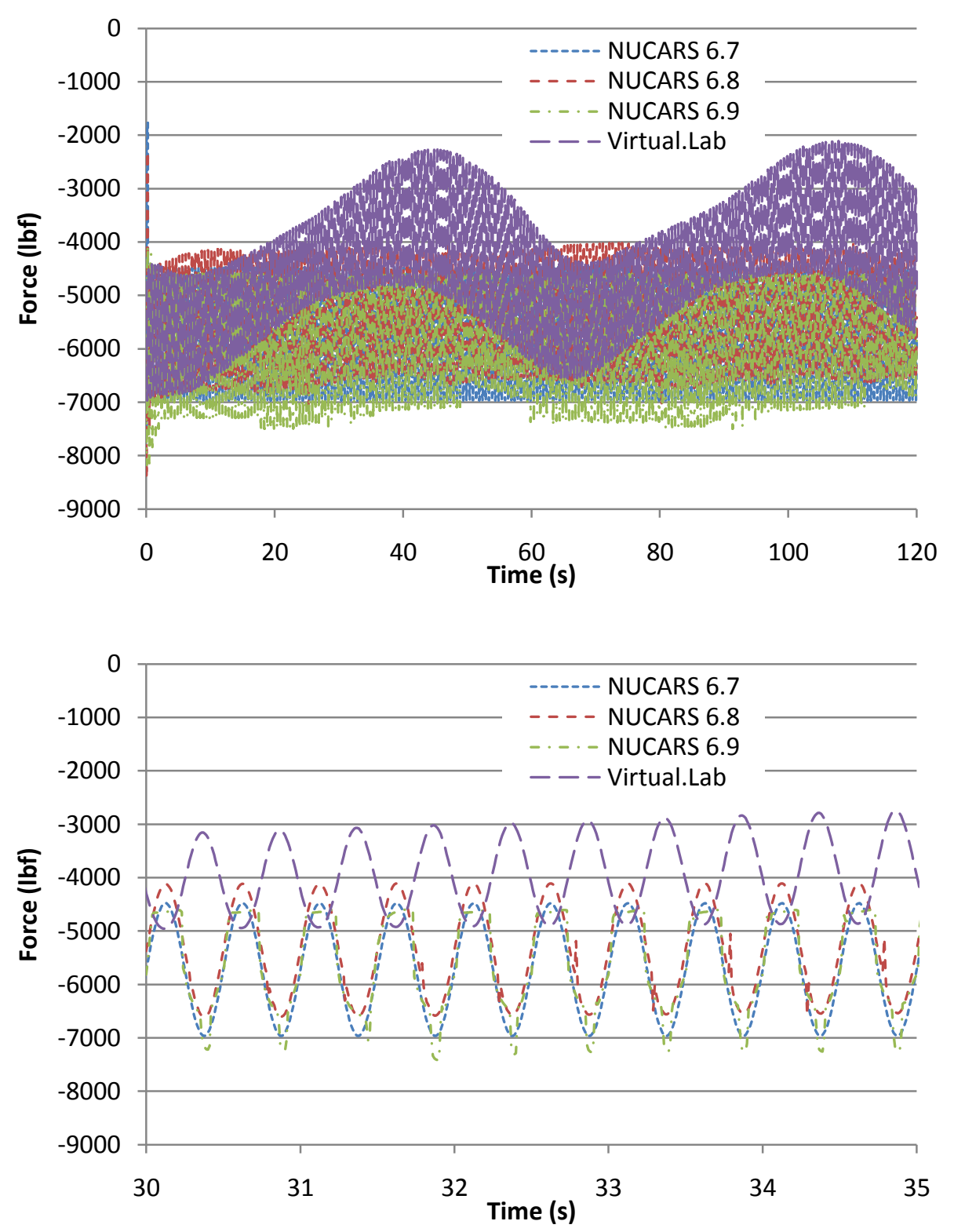

Figure 39. Vertical wedge force comparison between LMS $^{\circledR}$ Virtual.Lab model and NUCARS ${ }^{\circledR}$

The lateral force on the wedge is the force that acts perpendicular to the direction of the track. Figure 40 shows that the stand-alone model compares fairly well to wedge elements 6.7 and 6.9 , while 6.8 does not appear to follow the same pattern as the others. The variation in version 6.9 seems to be caused by the bounce input, while the stand-alone model experienced very minor lateral effects from the bounce. The variation in lateral force of the stand-alone model appears to be almost entirely from the warp input. 

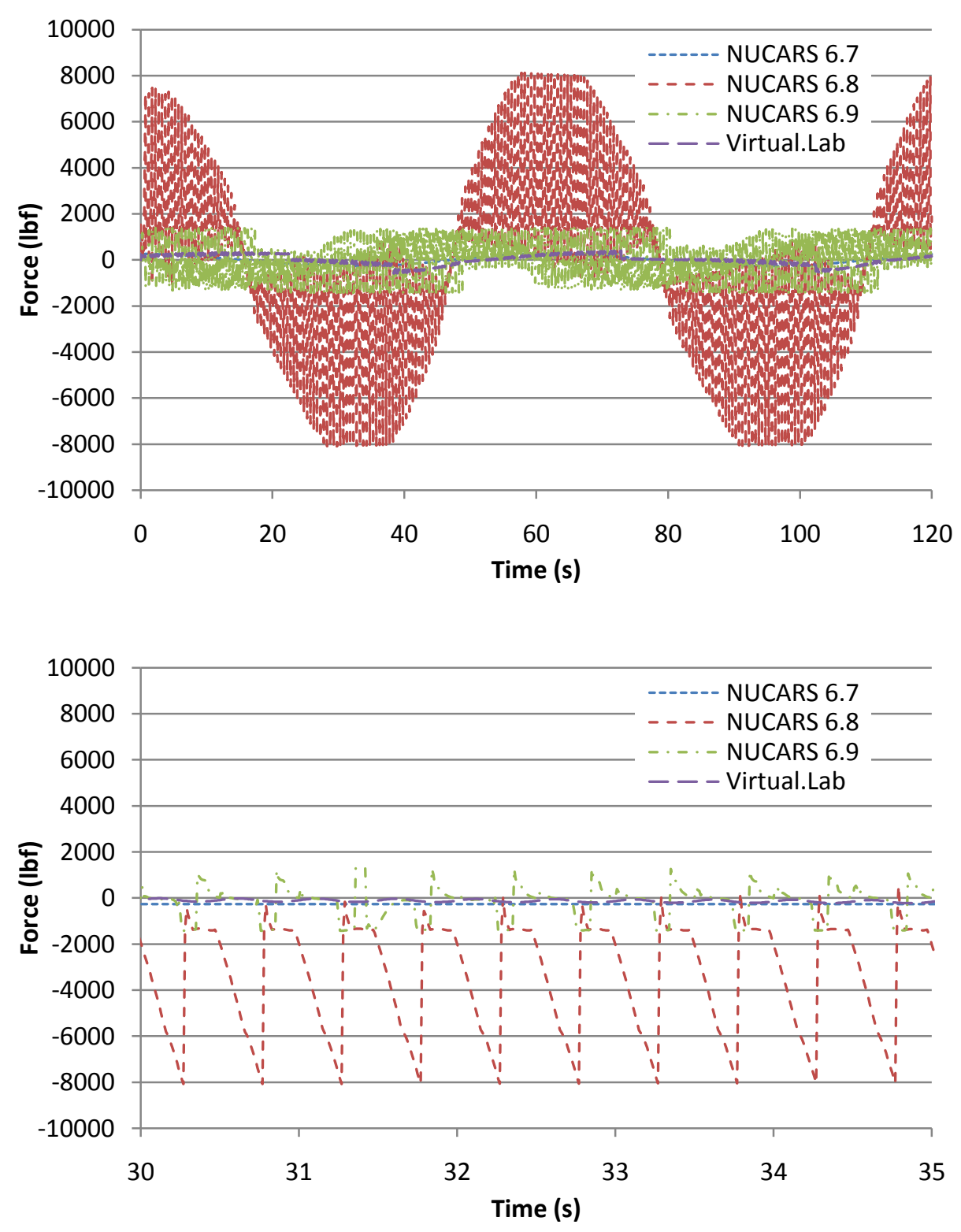

Figure 40. Lateral wedge force comparison between $\mathrm{LMS}^{\circledR}$ Virtual.Lab model and NUCARS ${ }^{\circledR}$

The longitudinal force of the wedge is an important factor in the friction wedges performance, because it is equivalent to the normal force for the friction between the wedge and the sideframe. As discussed in the NUCARS ${ }^{\circledR}$ section, versions 6.7 and 6.8 are not able to output the longitudinal force, so only version 6.9 was used for this comparison. Figure 41 shows the longitudinal force between the wedge and the sideframe. The longitudinal force on the stand-alone model varies as the warp angle changes, increasing and decreasing pressure on 
the wedge from the bolster, which is expected behavior, while the NUCARS ${ }^{\circledR}$ model's outputs did not behave as expected.
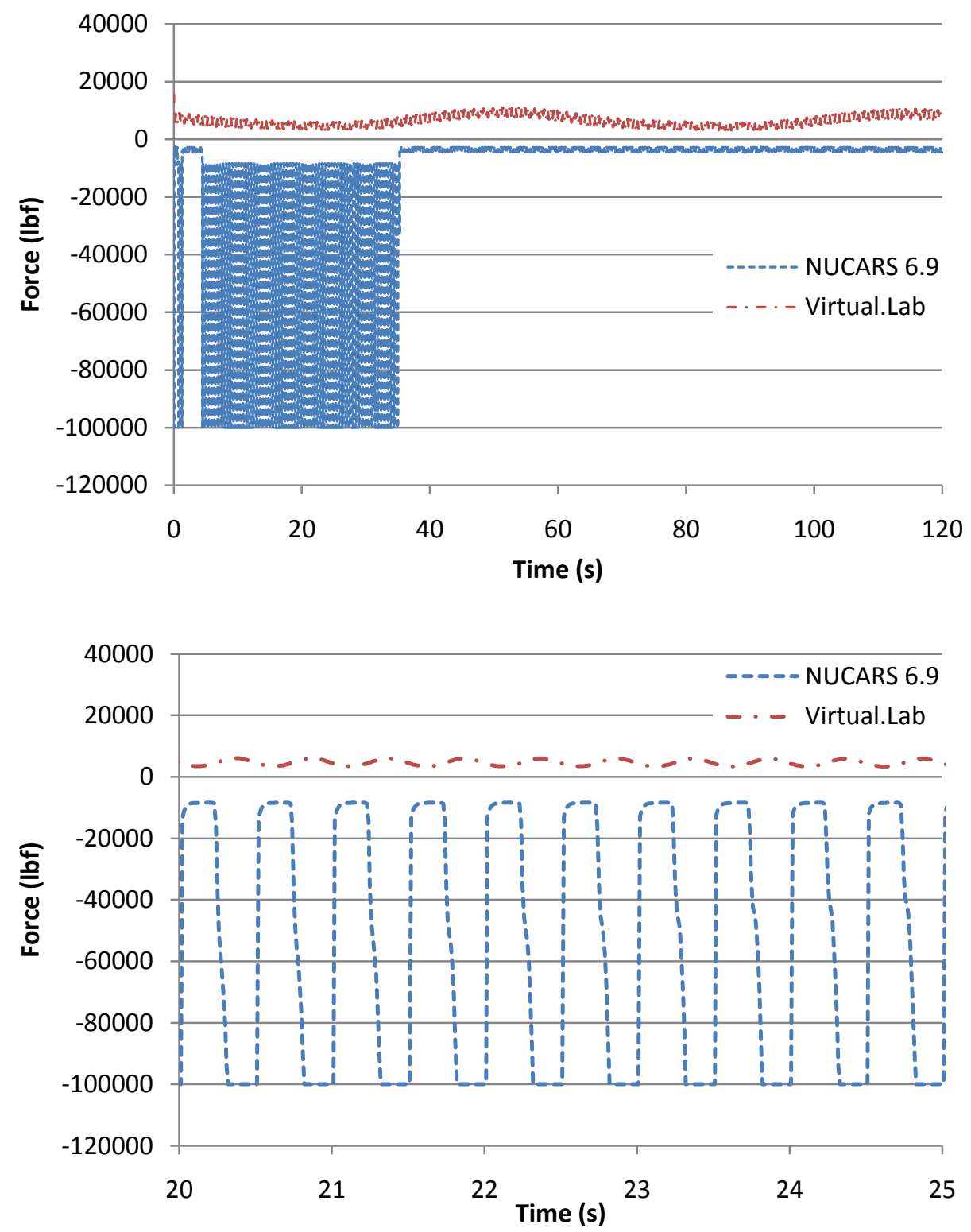

Figure 41. Longitudinal force comparison between $\mathrm{LMS}^{\circledR}$ Virtual.Lab model and NUCARS ${ }^{\circledR}$

With the benchmarking against the test data and NUCARS ${ }^{\circledR}$, variations of the warp and bounce inputs were used to investigate the combined effect of these inputs on the performance of the truck. Specifically we looked at the displacement, torque, and yaw of the bolster. In Figure 42 we can see that the amplitude of the bolsters displacement is similar for 
the pure bounce and warp/bounce inputs, with the warp/bounce oscillation centered on the displacement location from the pure warp case. Figure 43 shows that the warp stiffness was affected very little by the bounce input that was used in these simulations. It was also found that the bolster yaw angle was affected minimally by the bounce. Figure 44 show the yaw angle of the bolster from the two simulations.
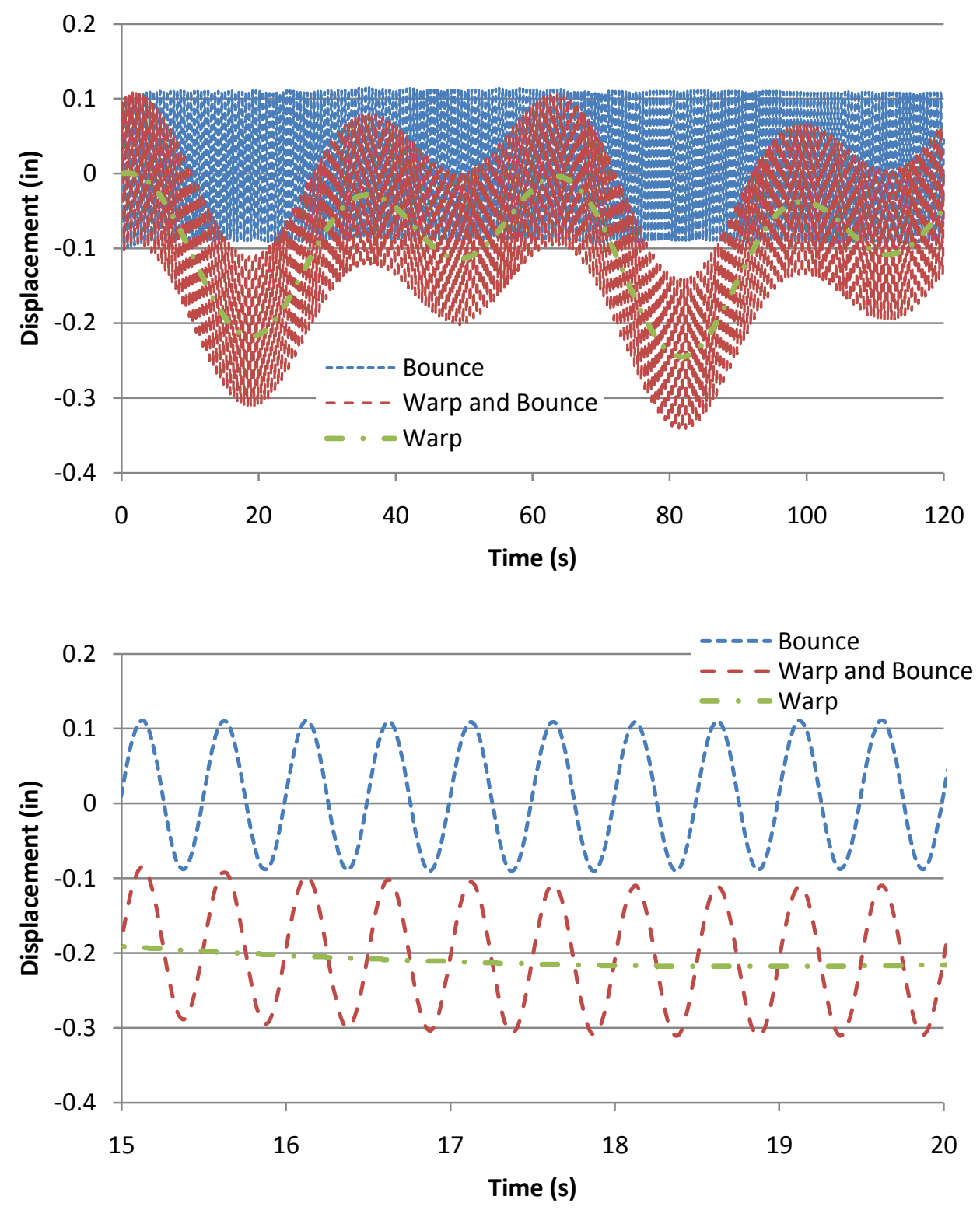

Figure 42. Bolster vertical displacement comparison between pure warp, pure bounce, and warp with bounce 


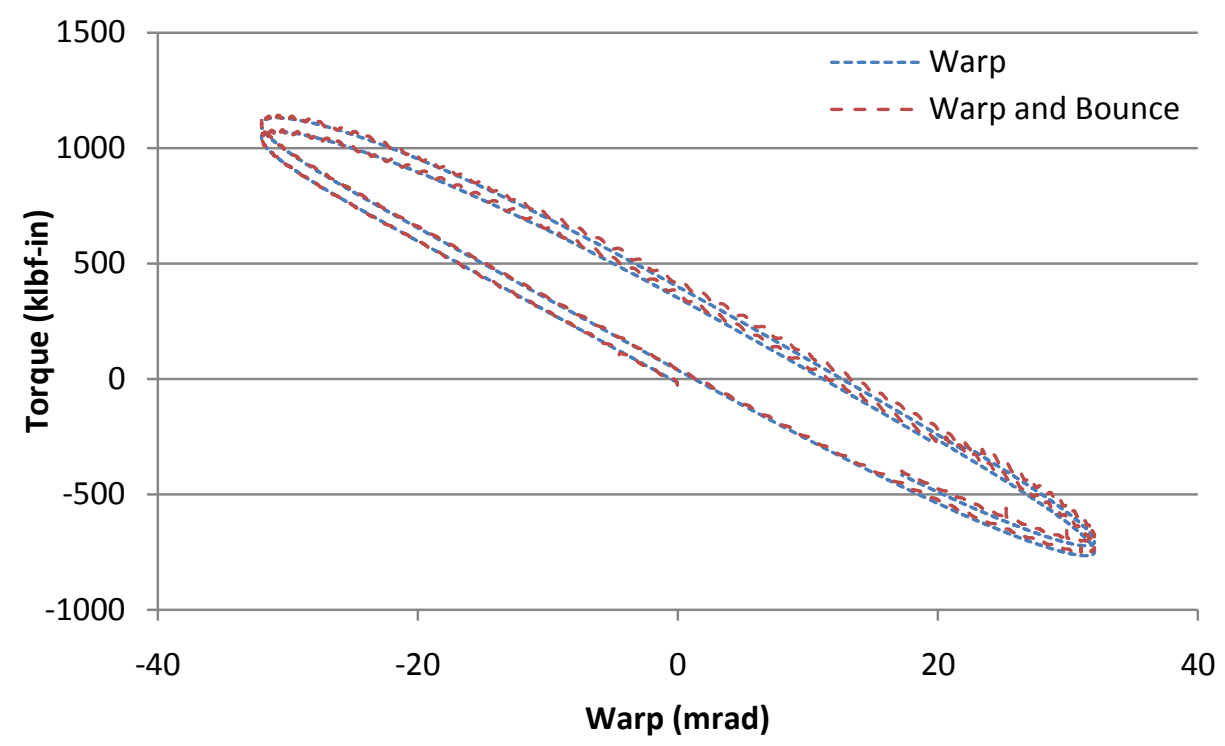

Figure 43. Warp stiffness comparison between pure warp and warp/bounce

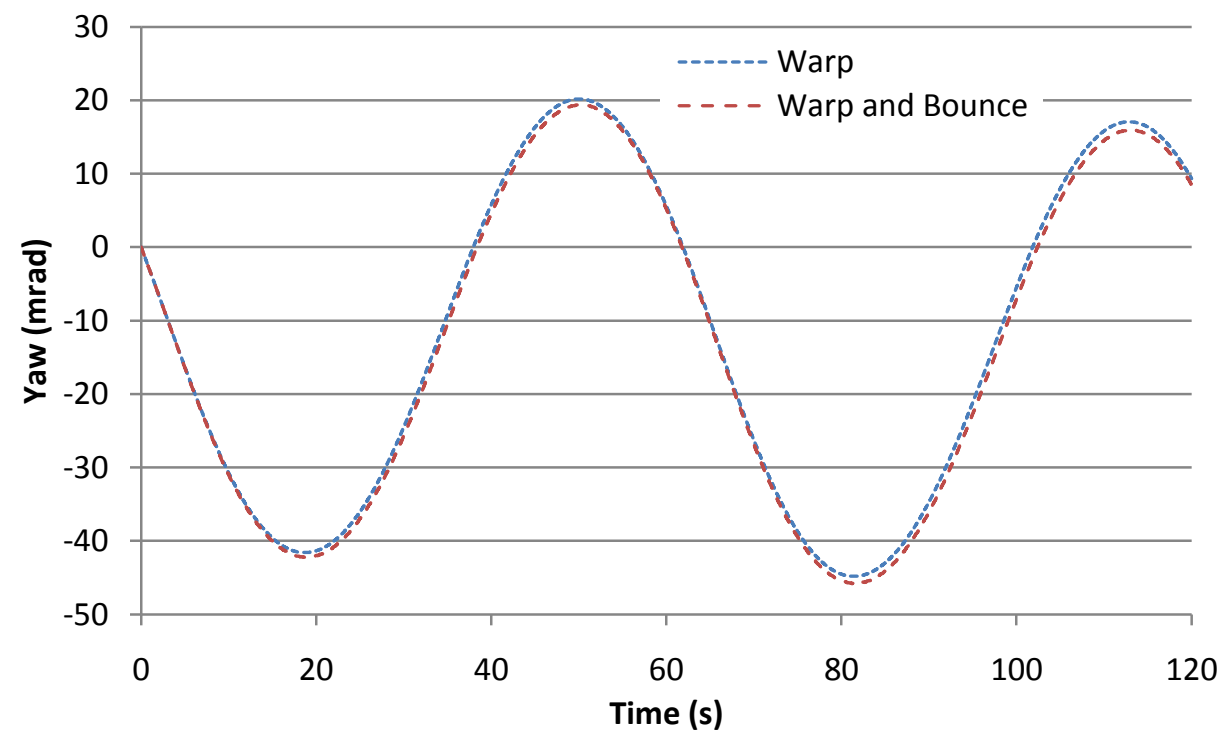

Figure 44. Bolster yaw comparison between pure warp and warp/bounce

Another point of interest was to see how the forces on the wedge are affected by the combination of warp and bounce. By plotting data from pure bounce against warp/bounce, and pure warp against warp/bounce, we can determine the effects of the interaction. For vertical force comparison between versions of the stand-alone model, this force is the vertical friction force between the sideframe and the wedge only, the spring force was not included. Figure 45 is a comparison of the vertical force on a wedge with a warp and a warp/bounce 
input. The plot shows that the bounce creates an oscillation in the force plot, centered fairly close to the force values for the pure warp case. The exception to this seems to be around the 20-30 seconds and 80-90 seconds, where the amplitude of this oscillation shrinks. This indicates an area where there is less vertical force available to damp the bounce of the bolster.
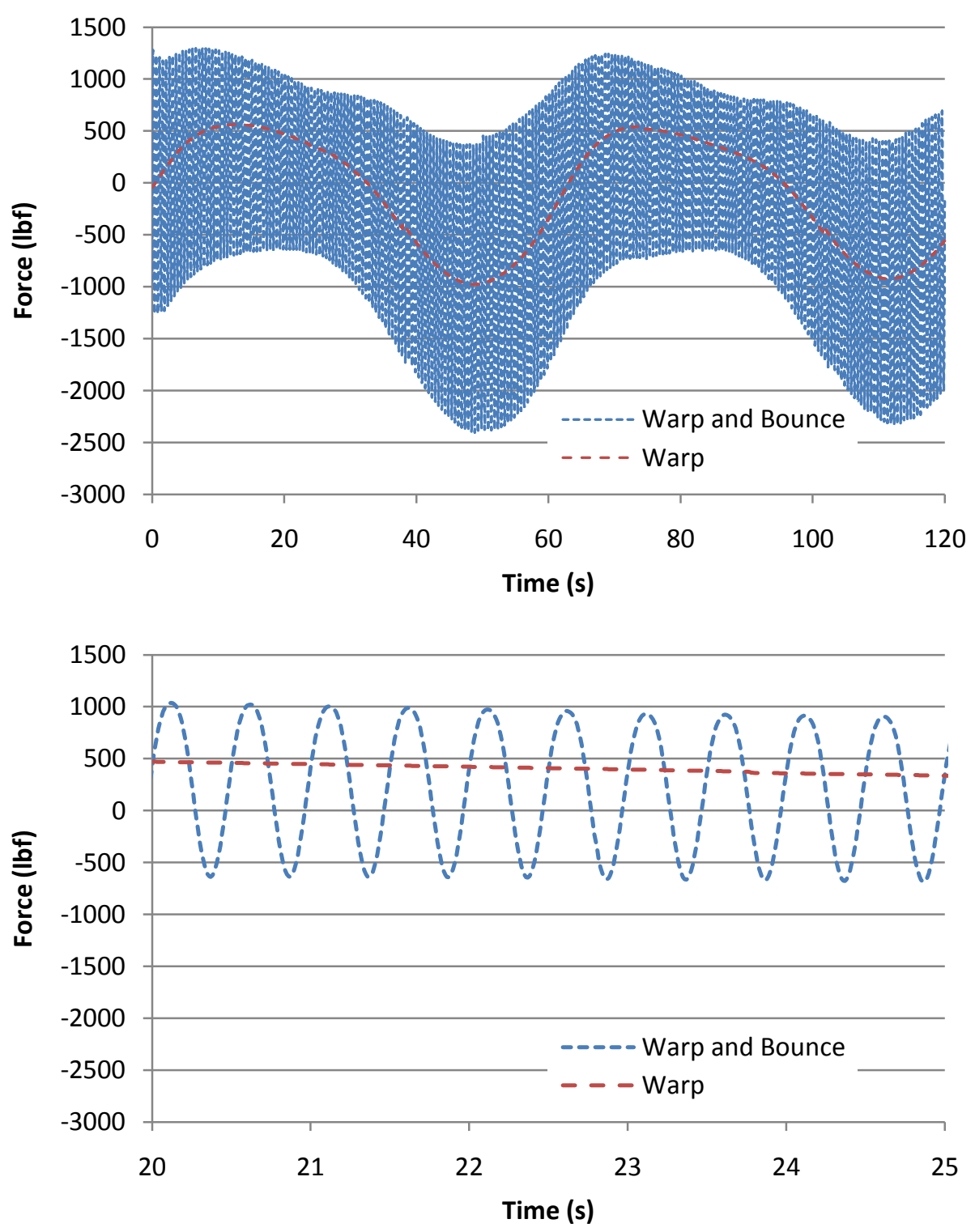

Figure 45. Wedge vertical force comparison between pure warp and warp/bounce

Figure 46 is a comparison between the vertical force on a wedge with a bounce and a warp/bounce input. The plot shows a significant decrease in the amplitude of the force as well as a decrease in the total force when the system is being both warped and bounced, which is 
especially evident in the sections previously mentioned. This indicated that the ability of the friction wedges to damp the system is diminished while the system is being warped.
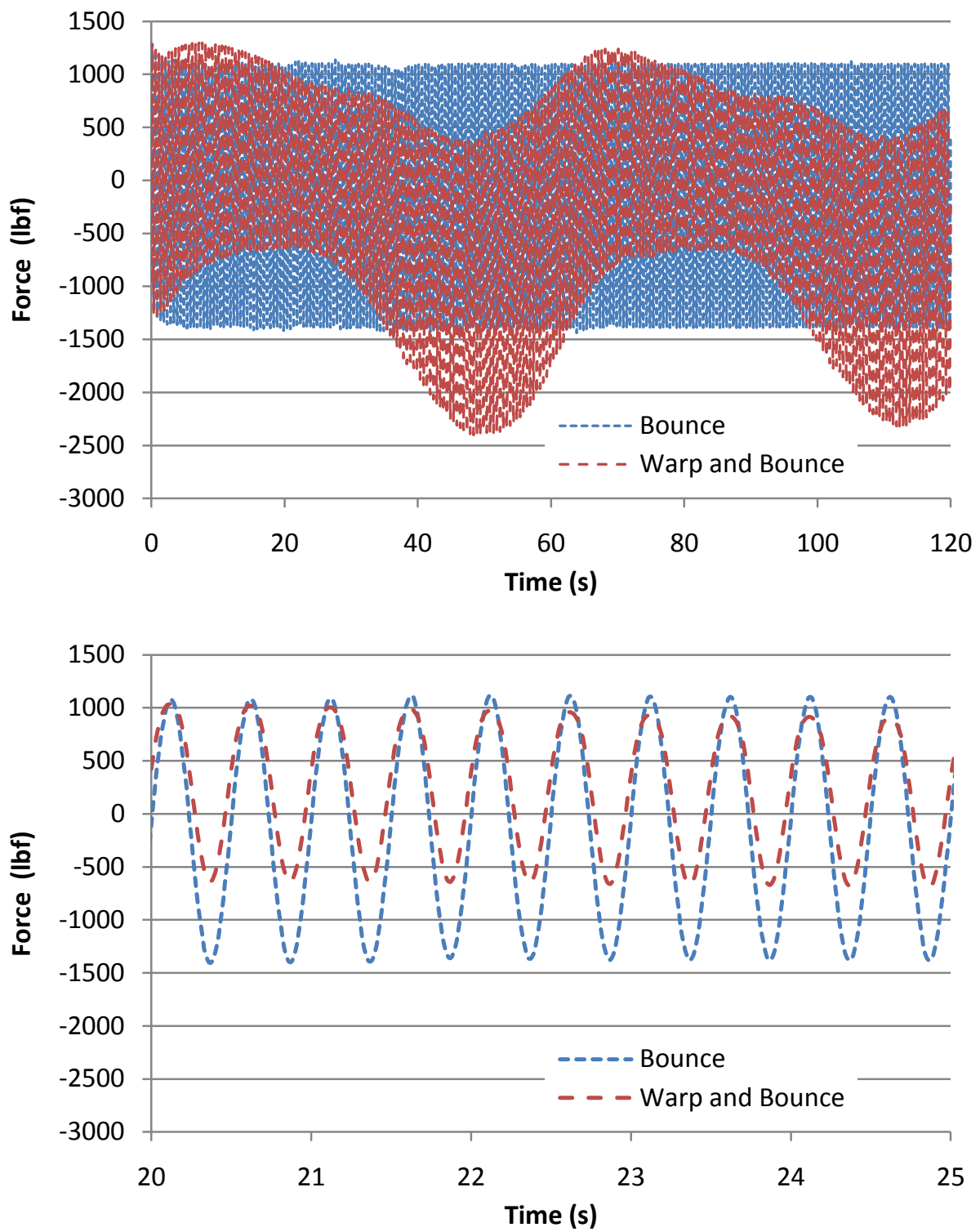

Figure 46. Wedge vertical force comparison between pure bounce and warp/bounce

The longitudinal forces were also plotted for the same cases. Figure 47 shows the comparison of longitudinal force between the pure warp and warp/bounce inputs. The plot indicates that the force from the warp/bounce case oscillates around the value of force for the warp case, with a relatively constant amplitude. Figure 48 shows the comparison of longitudinal force between the pure bounce and warp/bounce inputs. By comparing the 
longitudinal force plot to the vertical force plot, it was found that in the timeframe where the amplitude of the vertical force dropped, the amplitude of the longitudinal force also saw a drop. This is expected, as the vertical force is dependent on the longitudinal force, because it acts as the normal force in the friction equation.
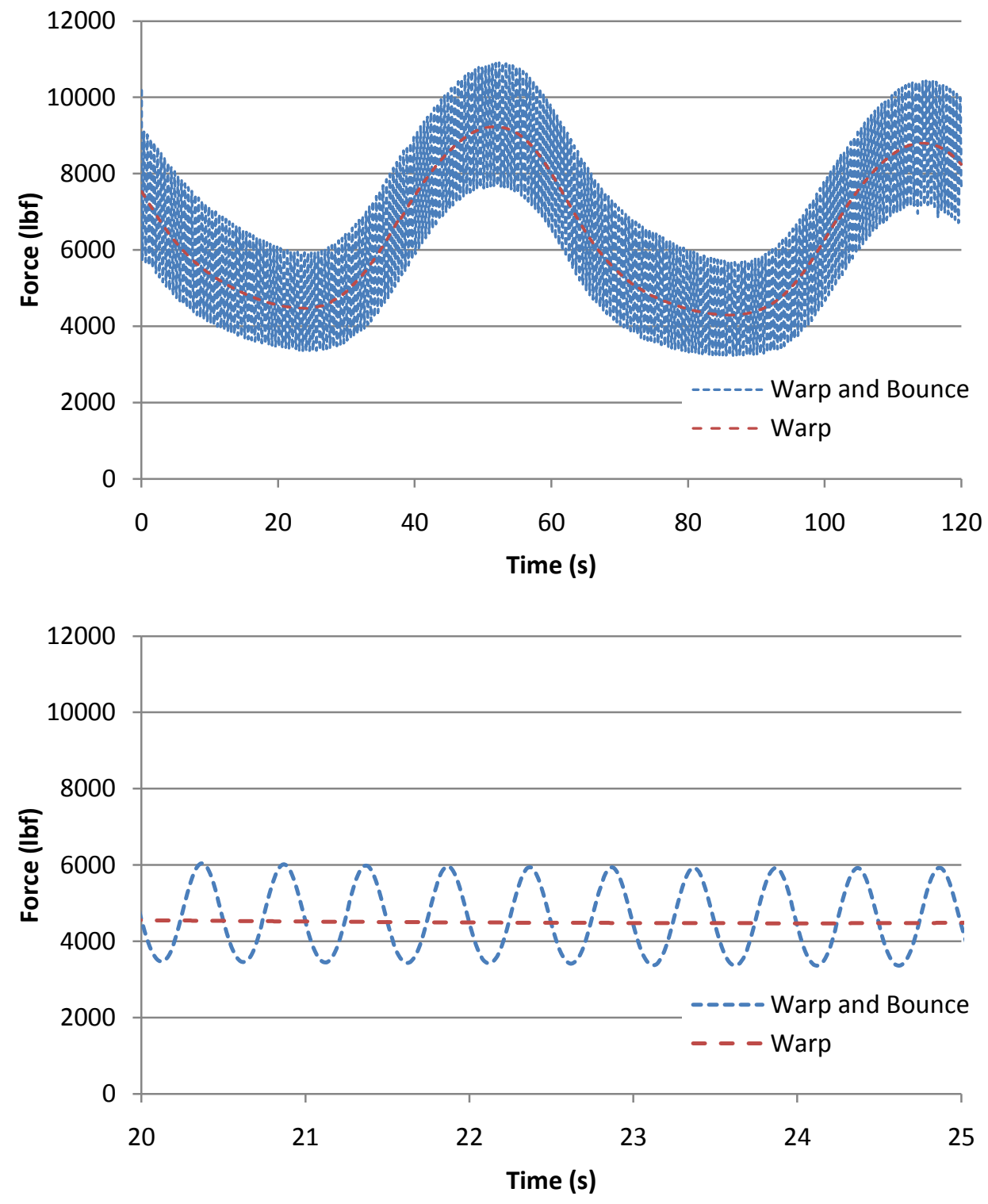

Figure 47. Longitudinal force comparison between pure warp and warp/bounce 

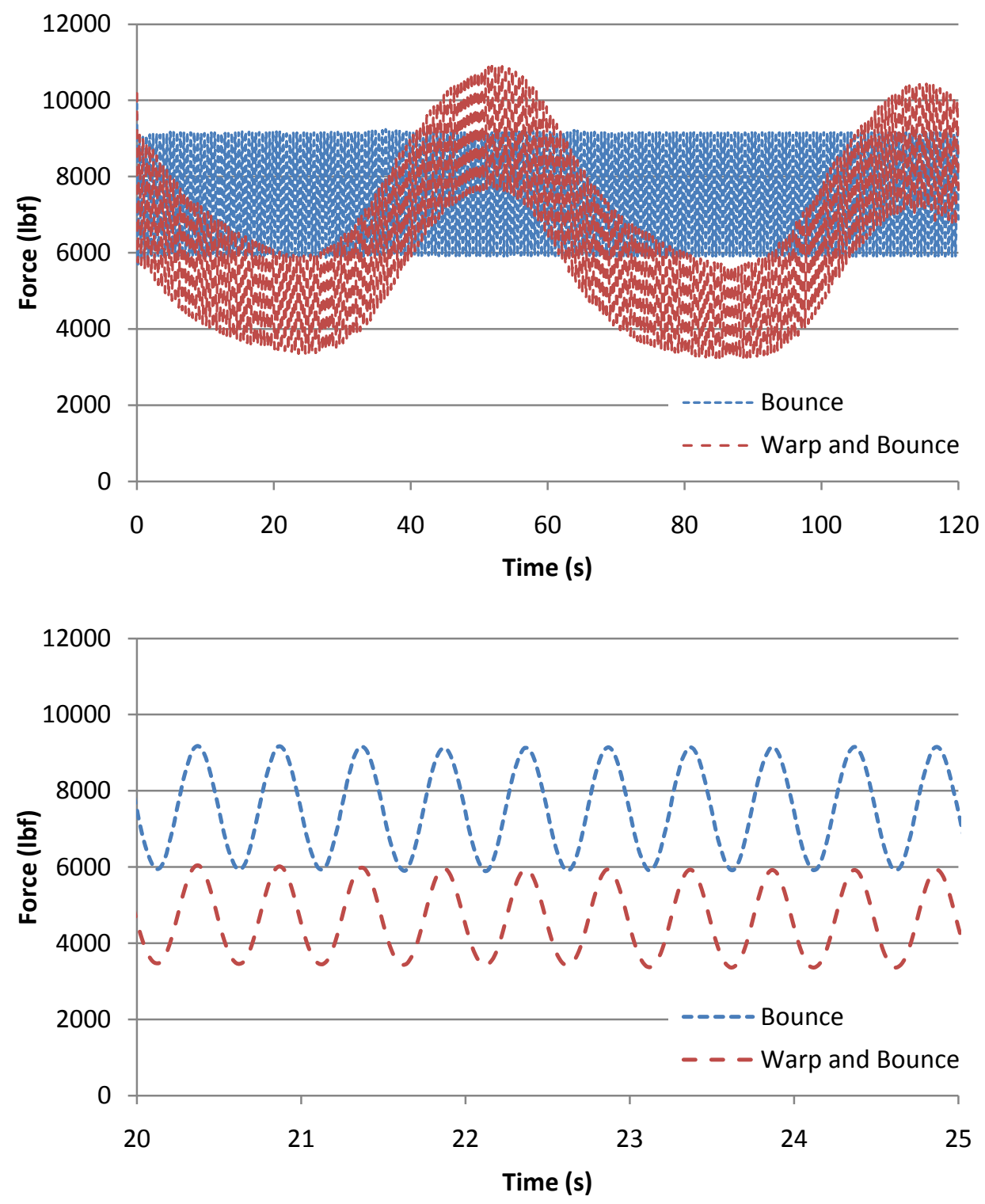

Figure 48. Longitudinal force comparison between pure bounce and warp/bounce 


\subsection{Full-truck Model with Worn Sideframes}

As with the standard full-truck model, a settling simulation was run to find the initial conditions of the bolster and wedges. Figure 49 shows the settling position of the bodies.

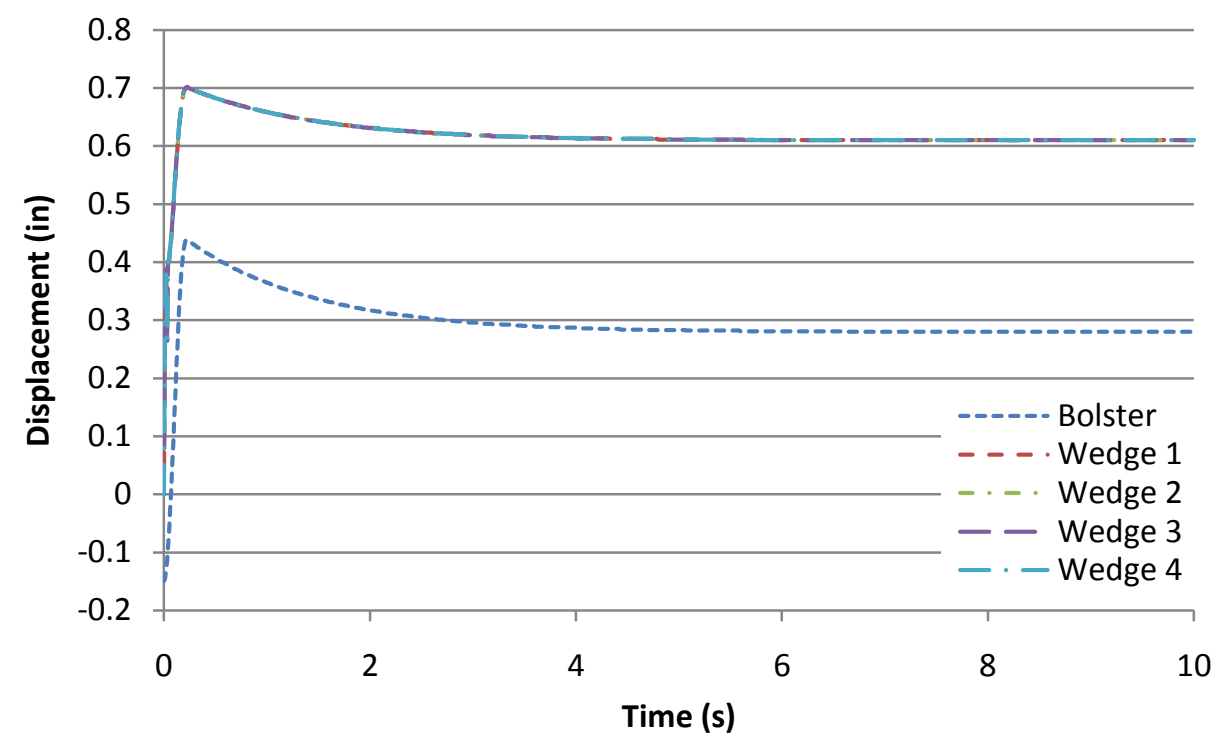

Figure 49. Bodies settling positions for the full-truck model with worn sideframes

Simulations were run on the full-truck model with worn sideframes with inputs to match the standard full-truck model, as there was no available test data to compare with. The first set of simulations was run with inputs of 0.5 inch bounce at $2.5 \mathrm{~Hz}$ on the sideframes. Figure 50 shows the displacements of the bolster and one of the wedges for both models. This plot shows that with the severely worn sideframes, the bolster and wedge are allowed significantly more motion than the unworn system. This indicates a dramatic decrease in the amount of damping the wedges provide. Figure 51 shows the pitch angle of the wedges during the bounce simulation. Only two of the wedges were plotted, because they were representative of the others. The plot shows that the wedges were able to pitch orders-of-magnitude more than in the unworn configuration. The pitch of both wedge 1 and wedge 2 in Figure 51 stays nearly constant around zero. This is because the wedge-sideframe interactions become almost like a ball joint for the worn wedges, allowing the wedges to rotate within the system. 


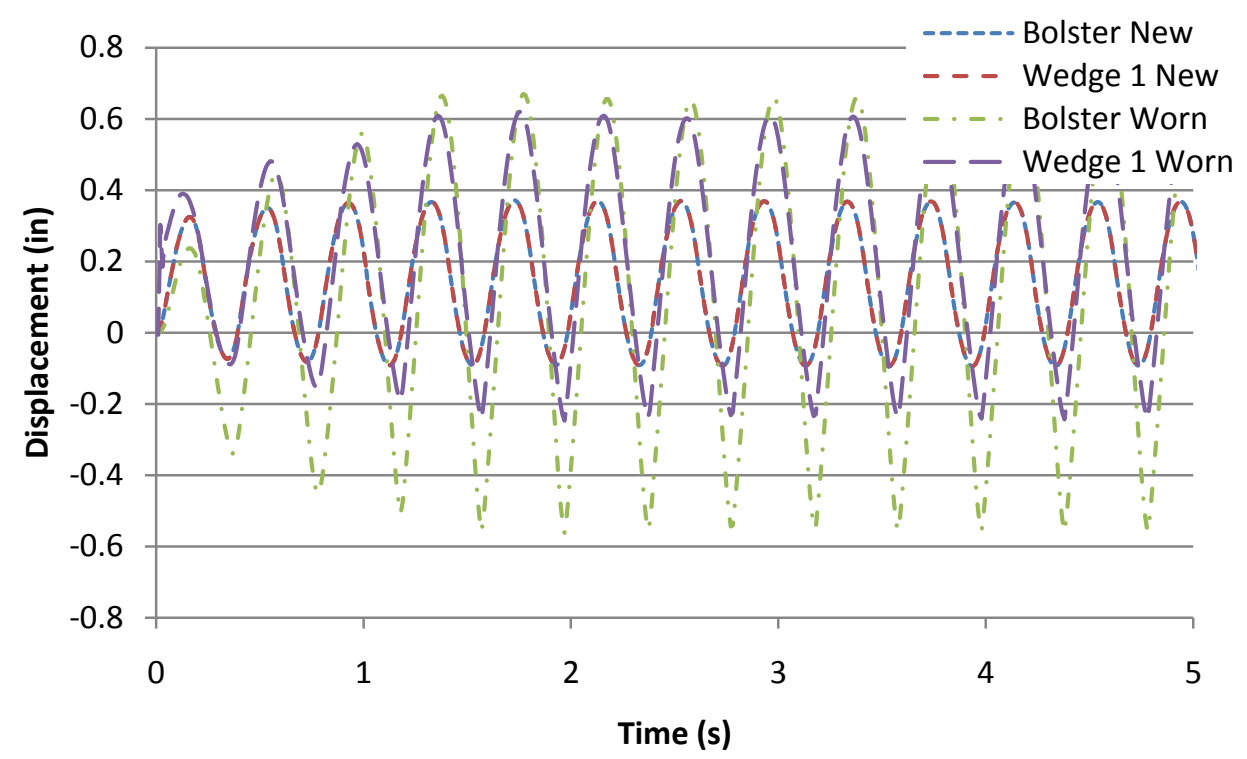

Figure 50. Displacement comparison between standard and worn models (bounce)

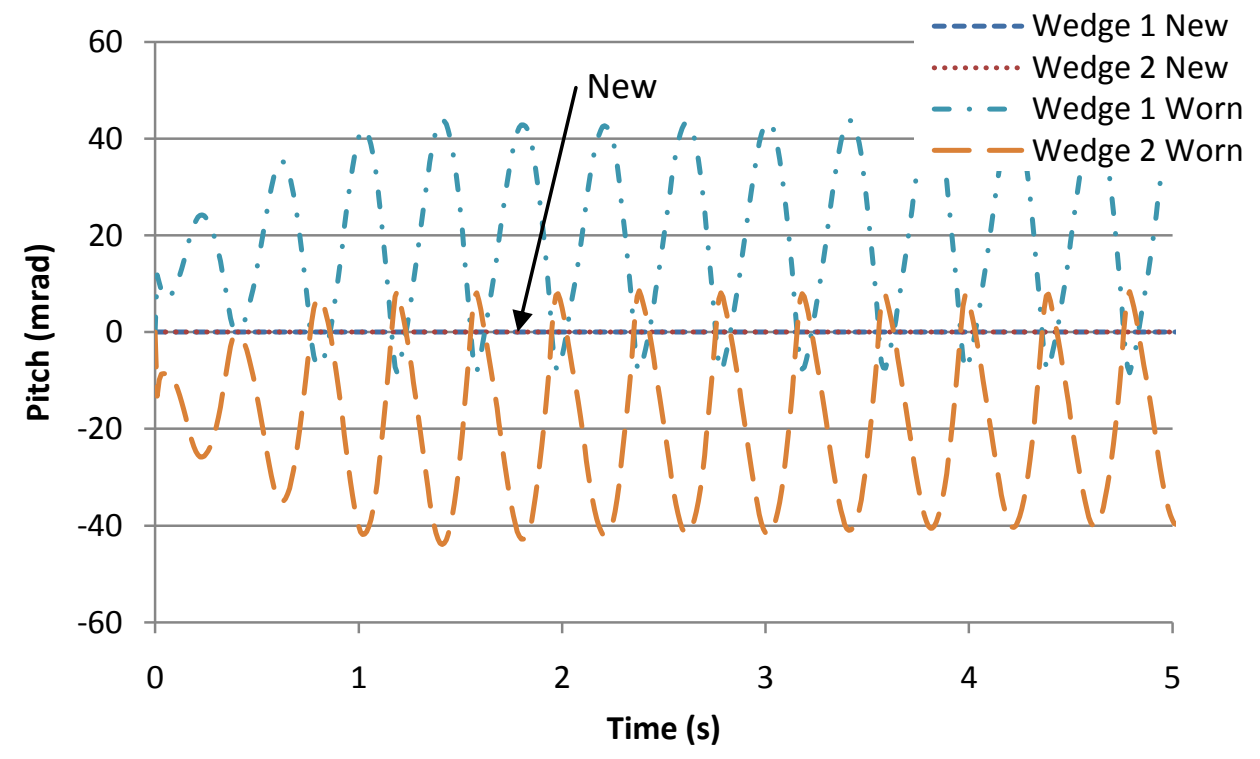

Figure 51. Wedge pitch comparison between standard and worn models (bounce)

By analyzing the forces on the wedge, we can better understand what happens throughout the bounce simulation. Figure 52 shows the vertical forces on the wedge, while Figure 53 shows the longitudinal forces. What these plots show is that for the worn model, the forces are lower until the very bottom of the wedge's displacement, where it is effectively getting stuck between the bolster and sideframe, causing a sudden large spike in force. 


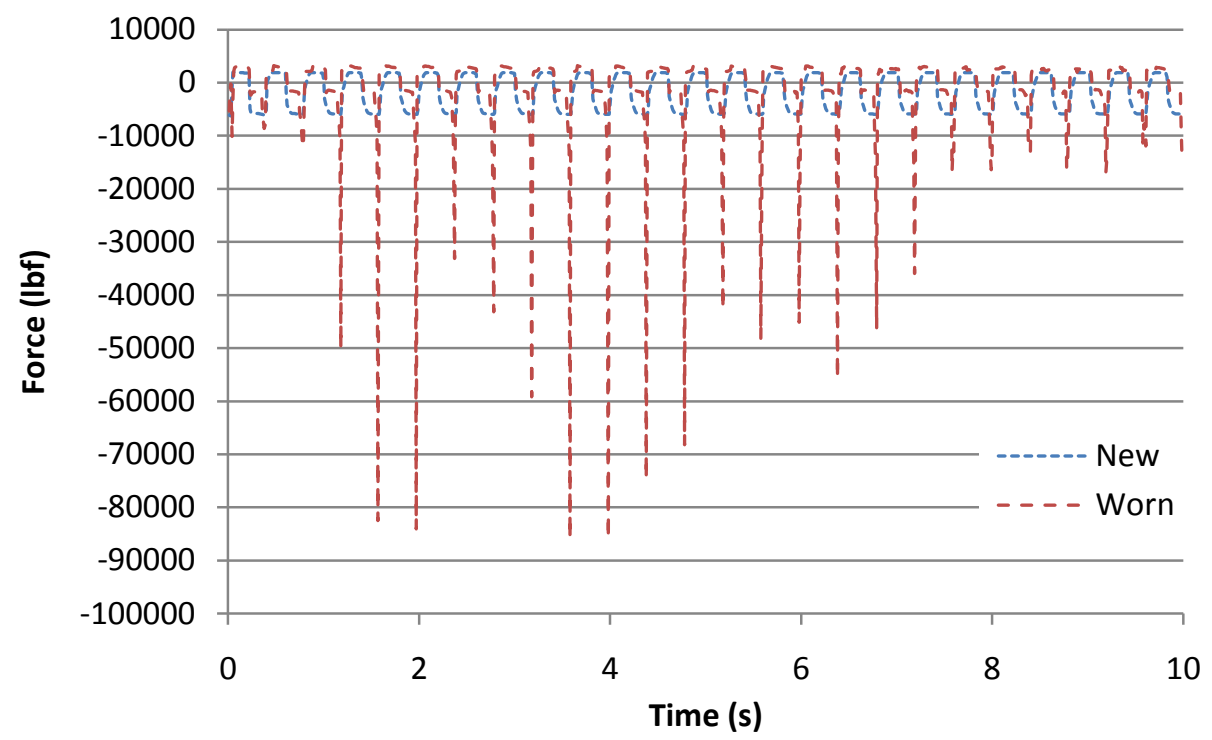

Figure 52. Vertical force comparison between standard and worn models (bounce)

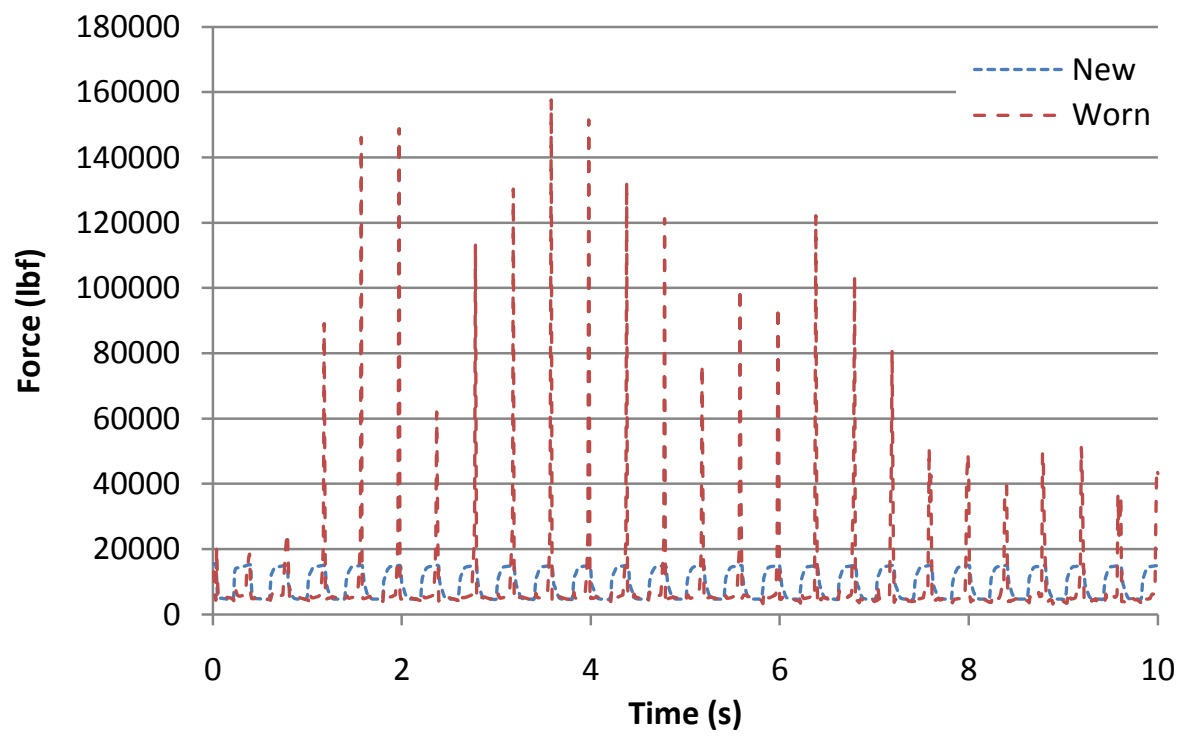

Figure 53. Longitudinal force comparison between standard and worn models (bounce)

The full-truck model with worn sideframes was then run to match the pure warp simulations run on the standard full-truck model. Figure 54 is a comparison of the warp stiffness between the two models. The plot shows that the worn model's warp stiffness is orders of magnitude lower than that of the standard unworn model. This indicates that without maintenance, the performance of the truck becomes greatly diminished. Figure 55 is a comparison of the bolster yaw angle for the two models. The worn model was able to keep the 
bolster more centered, which is a result of the wedge having a very loose fit between the bolster and sideframe. This loose fit meant that the forces were not large enough for the wedges to prevent the bolster from reaching its natural equilibrium point.

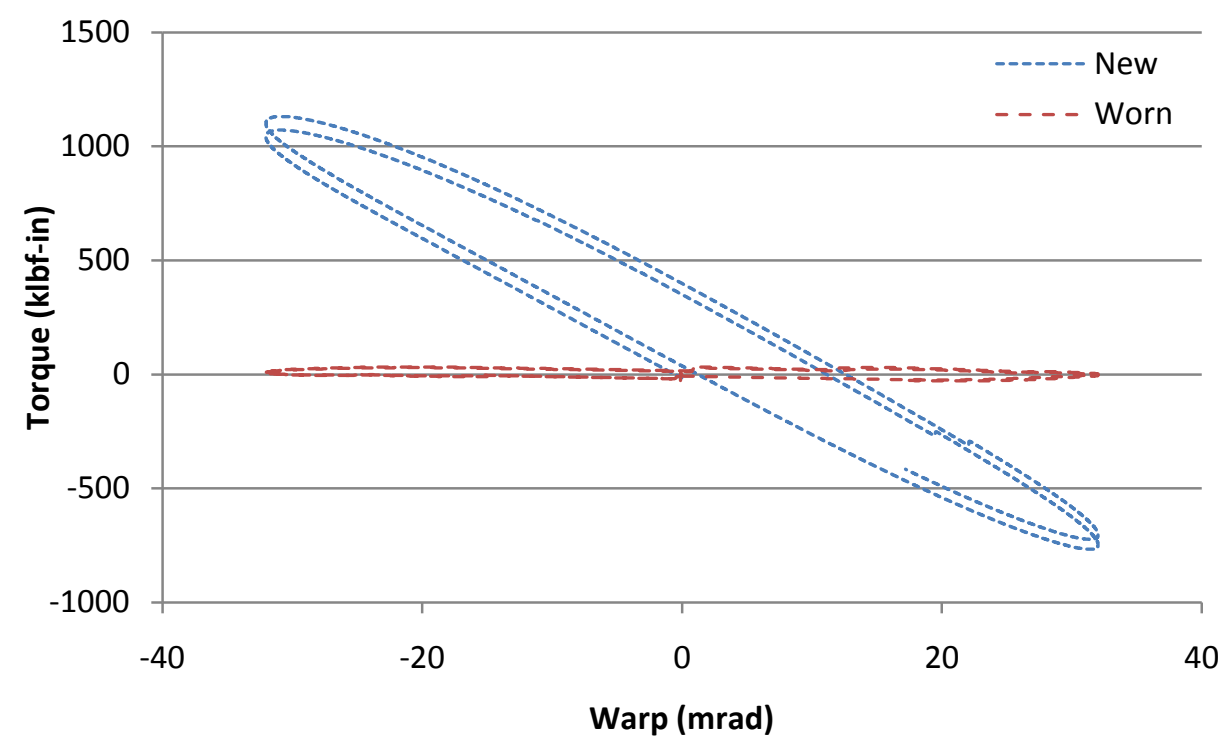

Figure 54. Warp stiffness comparison between standard and worn models (warp)

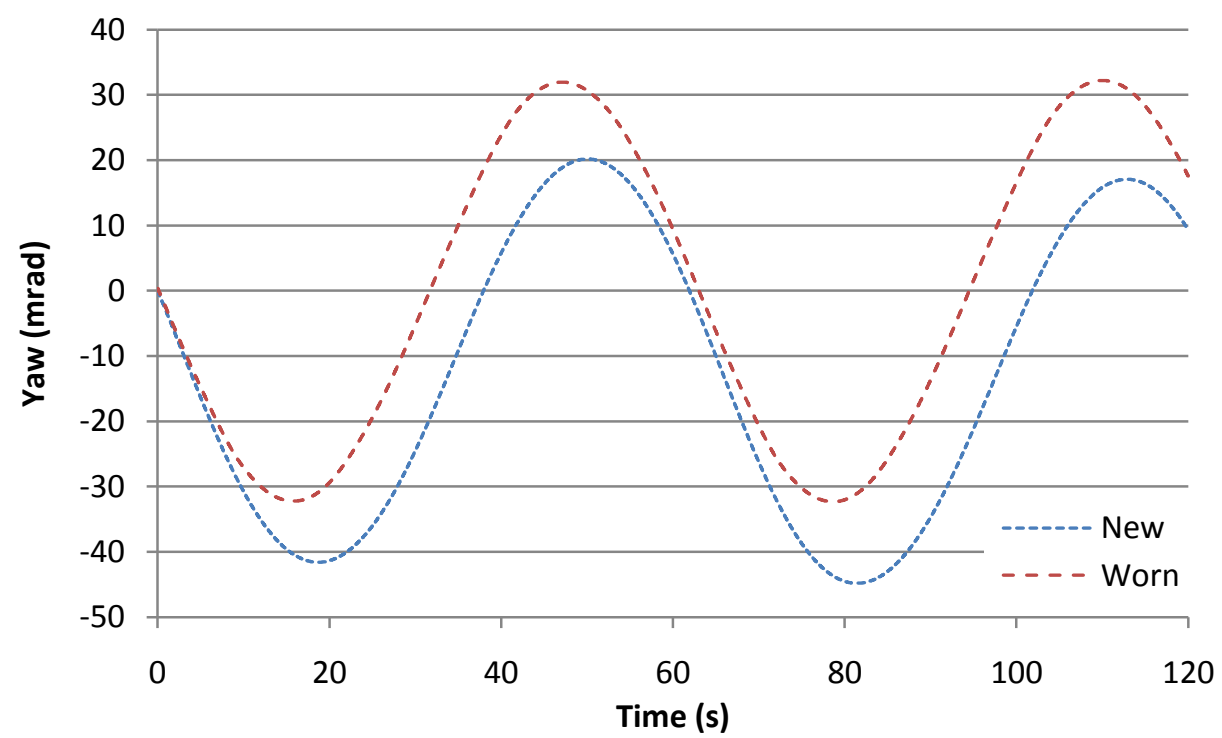

Figure 55. Bolster yaw comparison between standard and worn models (warp)

In Figure 56 we see that with the worn sideframe model, the frequency of the vertical force plot is about double that of the standard model. The same behavior is seen in Figure 57, which is a plot of the longitudinal force. These high peaks in force happen twice as much in the worn 
model due to the curved surface of the sideframe. At both the top and bottom of the motion, where the sideframe and bolster are closer together, the wedge is getting squeezed between the sideframe and bolster, and then some of the pressure is removed in the center of the motion, where the sideframe is further from the bolster. On the vertical force plot, we also see that the worn model does not exhibit the negative force on the wedge seen with the standard (new wedge) model. This is believed to also be an affect of the sideframe curvature, where there is a vertical contact force as well as the purely frictional force seen in the standard model.

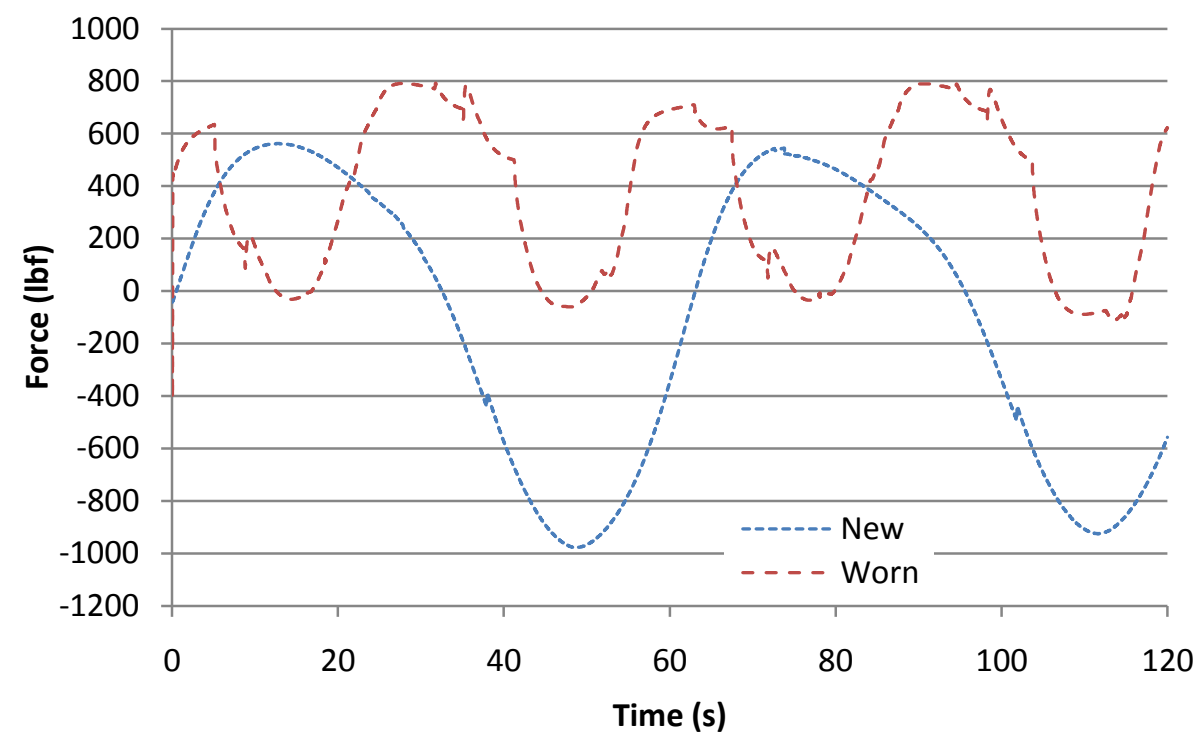

Figure 56. Vertical force comparison between standard and worn models (warp)

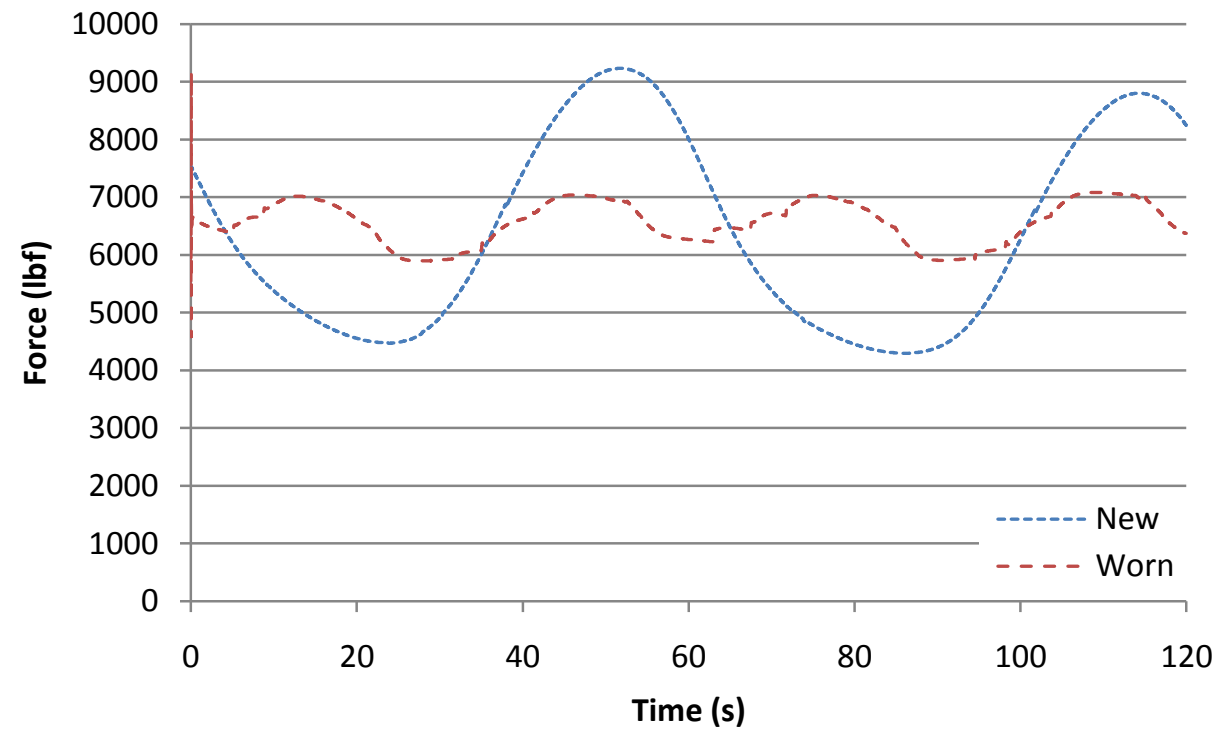

Figure 57. Longitudinal force comparison between standard and worn models (warp) 
A comparison between the pure warp, pure bounce, and warp/bounce inputs was not possible with this set of inputs, because the warp/bounce simulation reached an unsolvable state only seconds into the simulation. The contact points all have a maximum penetration depth, which corresponds to a maximum force on that point. With the drivers of the system being displacement controlled, one or more of the contact points reached that limit and stopped the simulation. In the stand-alone models, the maximum penetration depth was set at a depth that would correspond to a force many times greater than the weight of the car, so we feel it is reasonable to believe that the system would lock up well before that force was reached.

\subsection{Full-truck Model with Split-Wedges}

A settling simulation was run on the split-wedge model, as with the other models. Figure 58 shows the position of the bodies during the settling simulation.

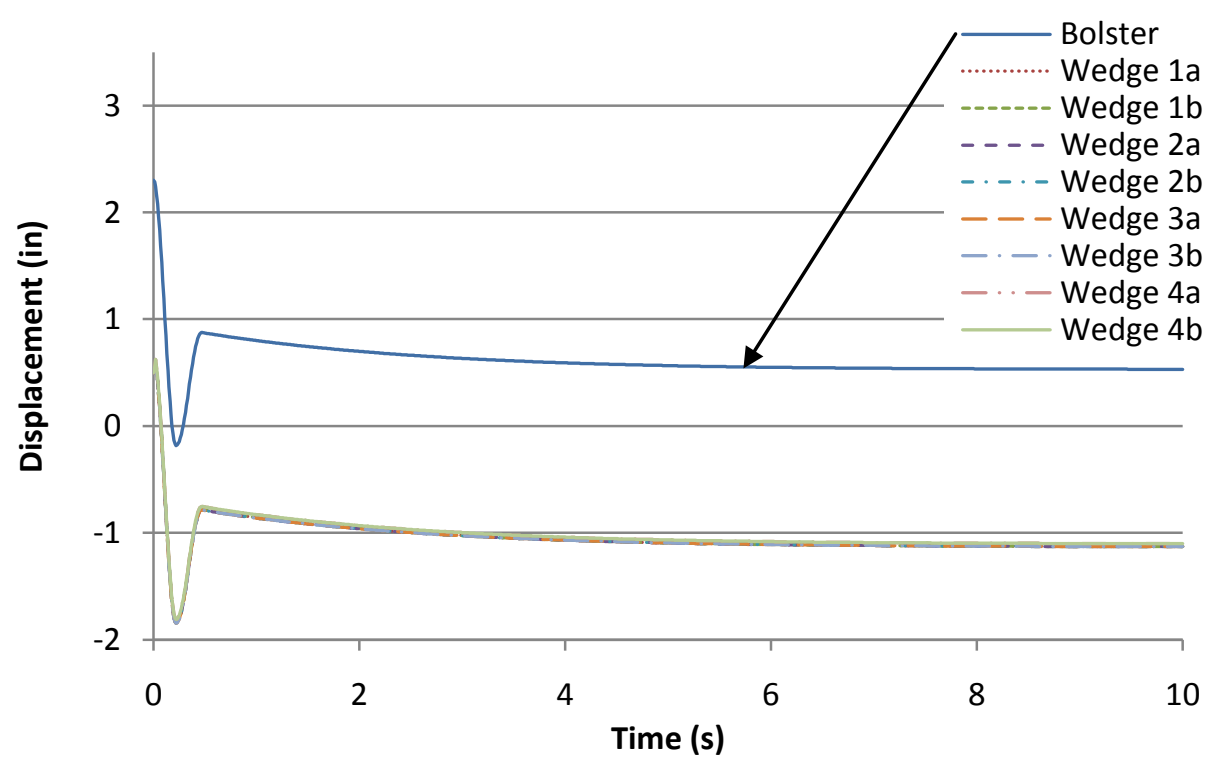

Figure 58. Bodies settling positions for the full-truck with split-wedges model

Simulations were run on the full-truck model with split-wedges with inputs to match the standard full-truck model, as there was no available test data for benchmarking. Similar to the worn model case, the first set of simulations was run with inputs of 0.5 inch bounce at $2.5 \mathrm{~Hz}$ on the sideframes. Figure 59 shows that the amplitude of displacements of the wedges and 
bolster are very similar for both the standard and the split-wedge models for a bounce input. The slight offsets seen in the plot are a product of the geometry and initial conditions of the bodies. In Figure 60 we see that the pitch of the wedges is very similar between the two models. This would be expected as both models have flat surface contact between the wedge and sideframe, allowing for little pitch.

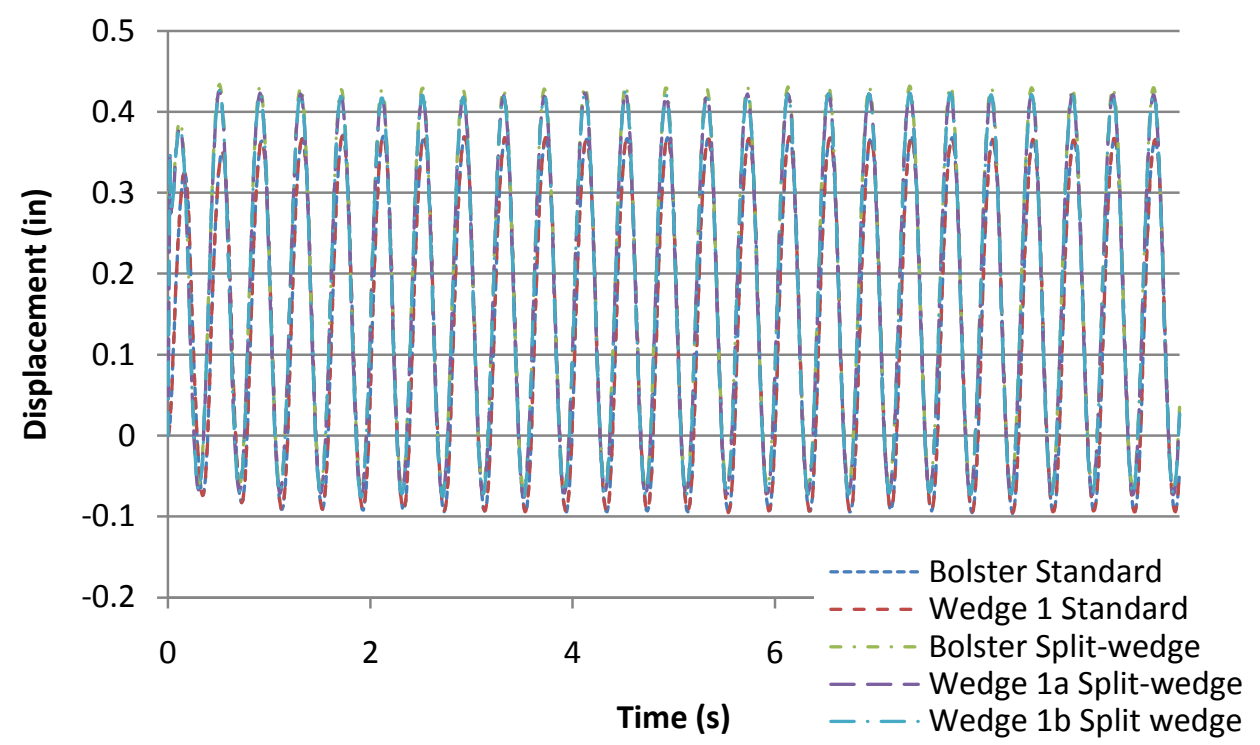

Figure 59. Displacement comparison between standard and slit-wedge models (bounce)

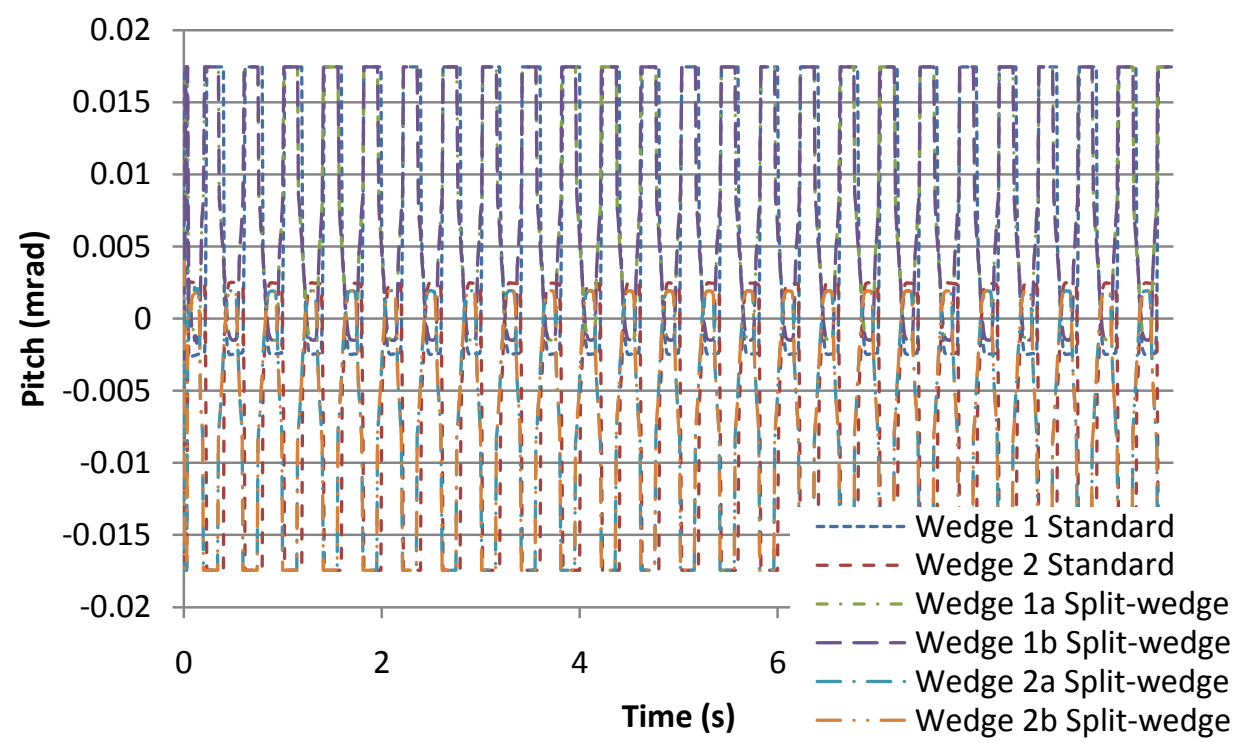

Figure 60. Pitch comparison between standard and split-wedge models 
As with the other parameters discussed, the forces on the wedge are similar for the standard and split-wedge models. In Figure 61 we see that the overall amplitude of force is slightly greater on for the split wedge model. This can be explained by the larger longitudinal forces seen in Figure 62. The longitudinal force plot show that the split-wedge model sees more force at all times, which is because the settling position of the system was slightly lower, causing the springs under the wedges to exert more force.

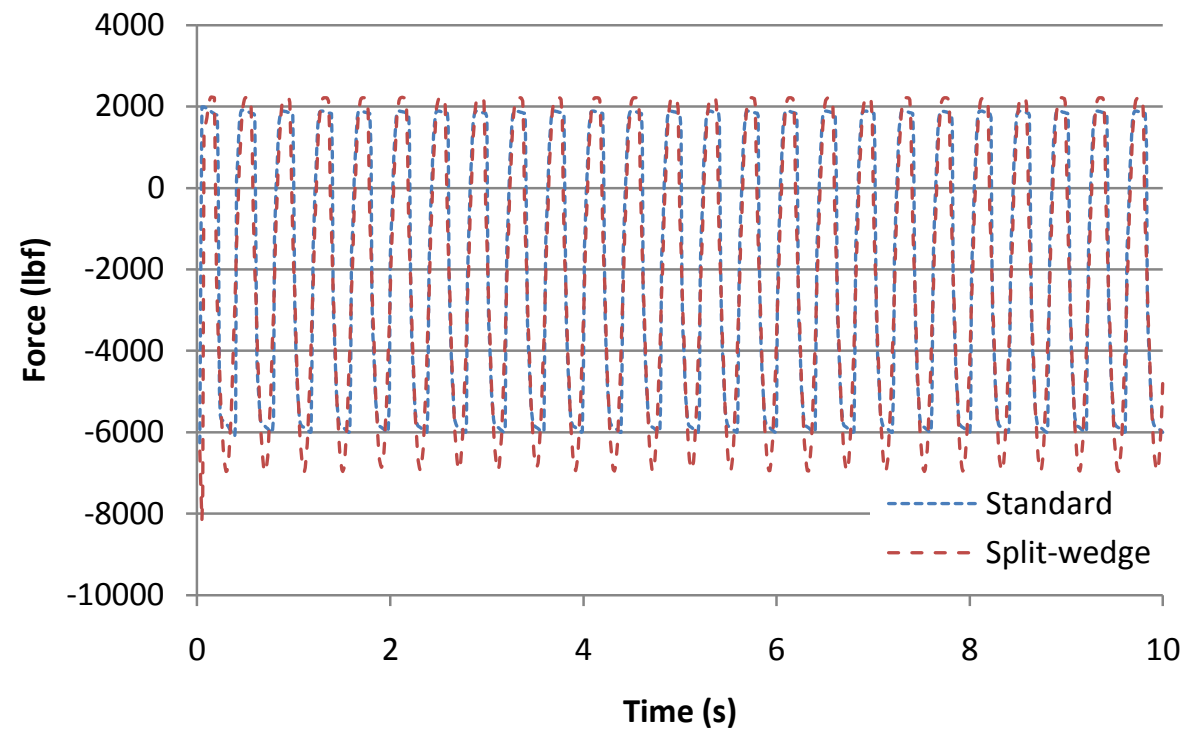

Figure 61. Vertical force comparison between standard and split-wedge models

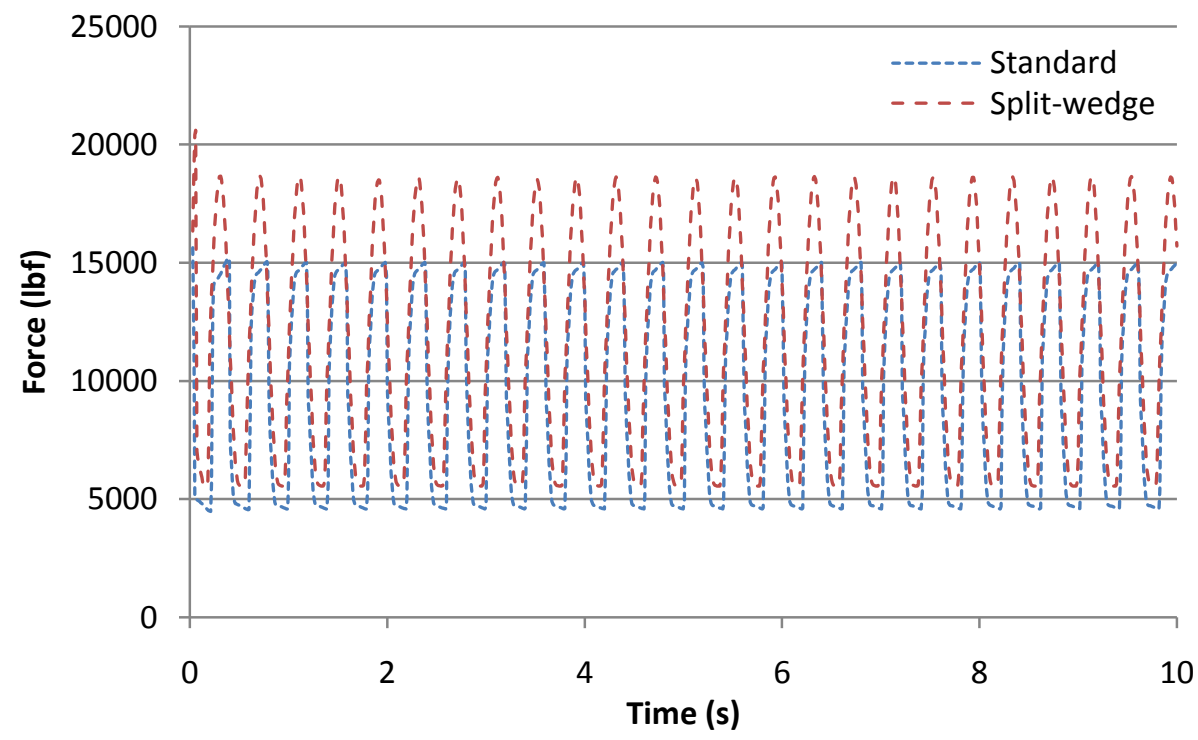

Figure 62. Longitudinal force comparison between standard and split-wedge models 
The inputs of the model were then set to match those of the full-truck model warp/bounce simulation. Figure 63 shows the warp stiffness of the split-wedge and standard model. The interesting thing to note about this plot is that the warp stiffness of the split-wedge model stays almost constant for each direction of warp. We believe that the reason for this behavior is due to the wedges yawing, so that only one edge was in contact with the sideframe. This is due to the interaction between the bolster and the wedge, in which each of the wedges in the pair get stuck in their respective $V$-shaped pocket of the bolster. Since the two halves are constrained by the way the spring connection was created in this model, when one of the wedges is being pushed into the bolster pocket, the other is also constrained to move into the bolster pocket. This effectively constrains the wedge to yaw with the bolster. In Figure 64 we see that the split-wedges keep the bolster centered better than the standard wedges. This is a result of the wedges being stuck in the pocket. By not allowing the wedges to rise as much with respect to the bolster, the bolster more closely follows the warp input from the sideframes.

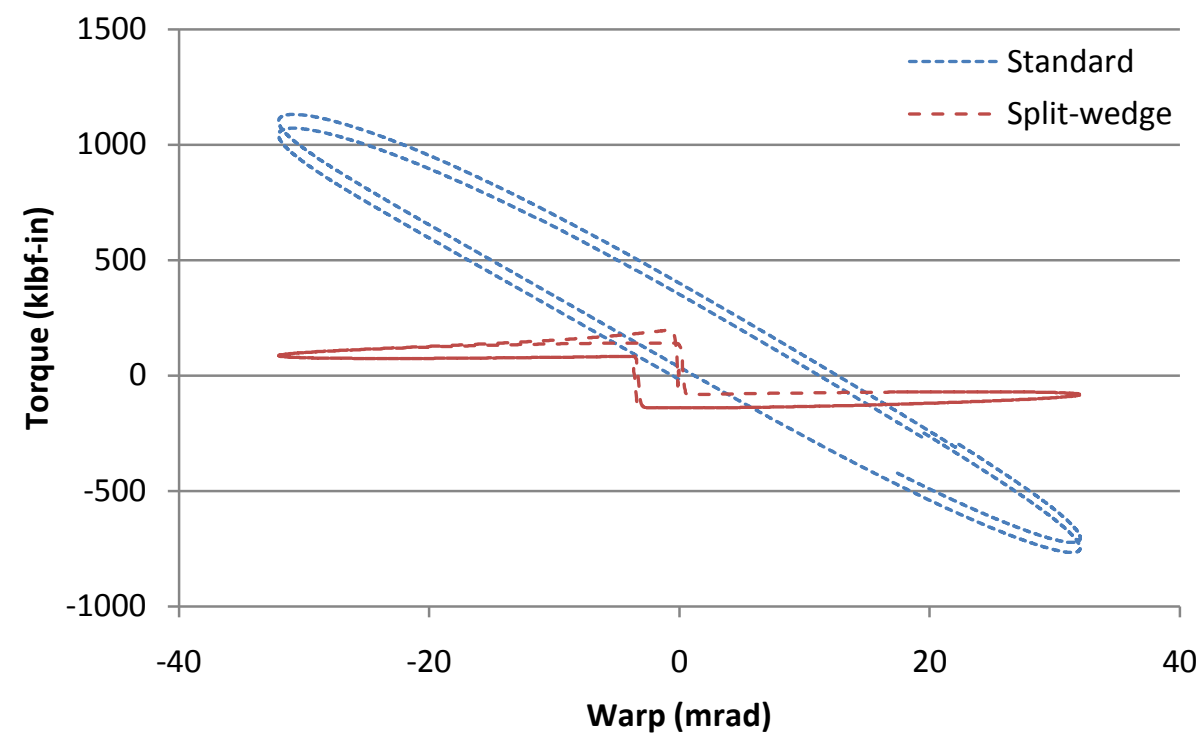

Figure 63. Warp stiffness comparison between standard and split-wedge models (warp) 


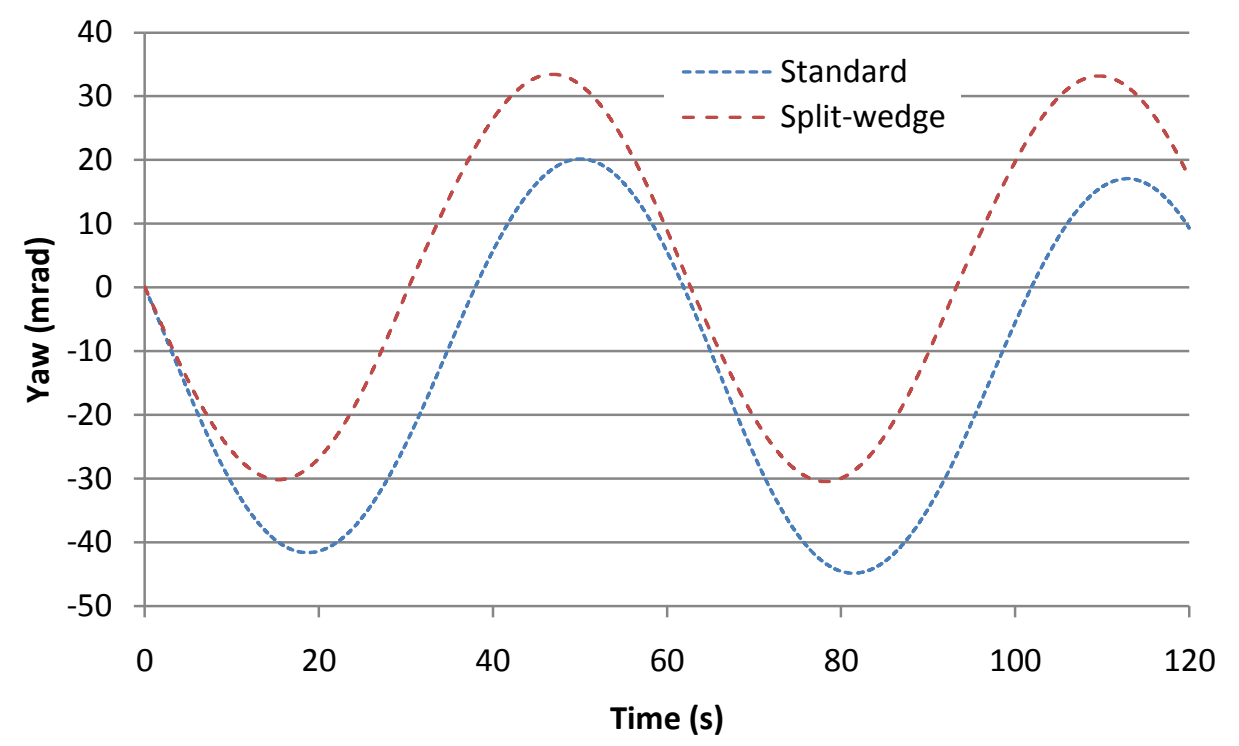

Figure 64. Bolster yaw comparison between standard and split-wedge models

When we compare the forces on the split-wedge system versus the standard wedge system we find a distinct difference in behavior. It is important to note, that the forces shown are for both wedges in the split wedge pair. In Figure 65 we see that the slit-wedges do not exhibit the same smooth force loading behavior as the standard wedge. Most notably is the spikes around 30 and 90 seconds. These spikes occur when the warp causes the force to shift from one wedge to the other.

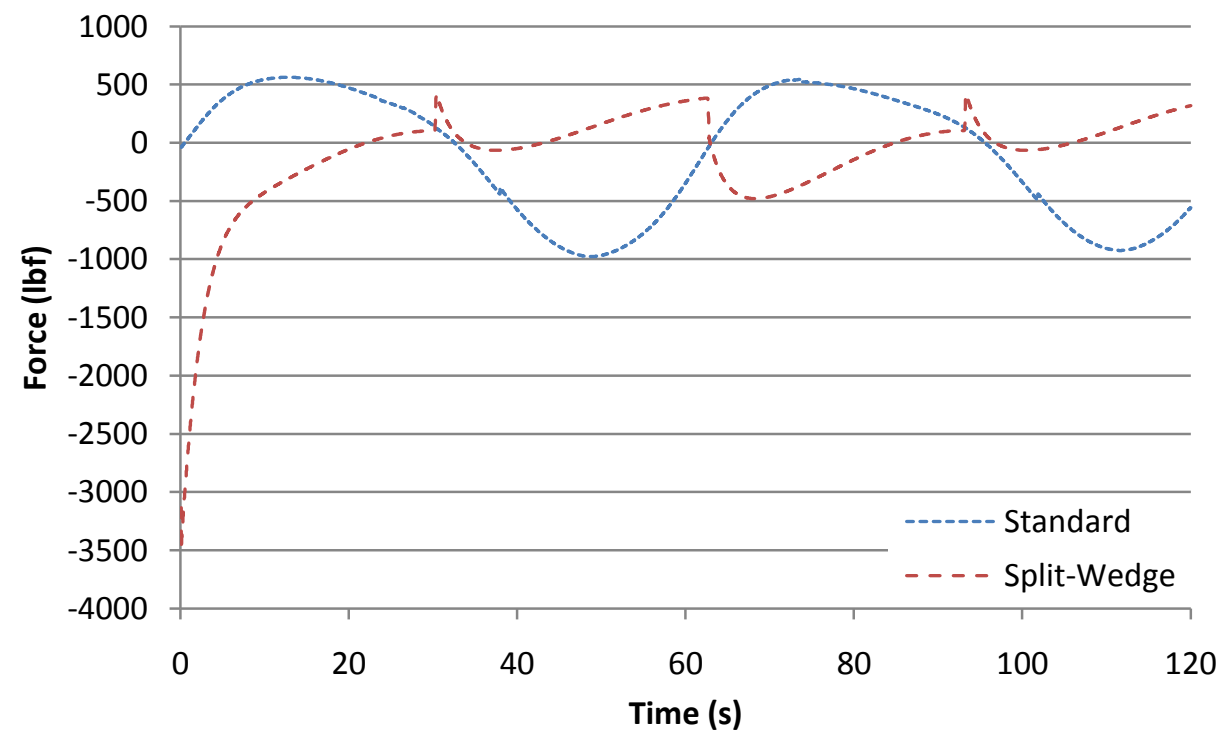

Figure 65. Vertical force comparison between standard and split-wedge models 
In Figure 66 we also see that the forces rise during this occurrence. The longitudinal force also differs in that while the warp is pushing the bodies in the opposite direction than the wedges, there is not a large decrease in force on the wedge pair. This is a product of the contact geometry between the bolster and the wedge pair.

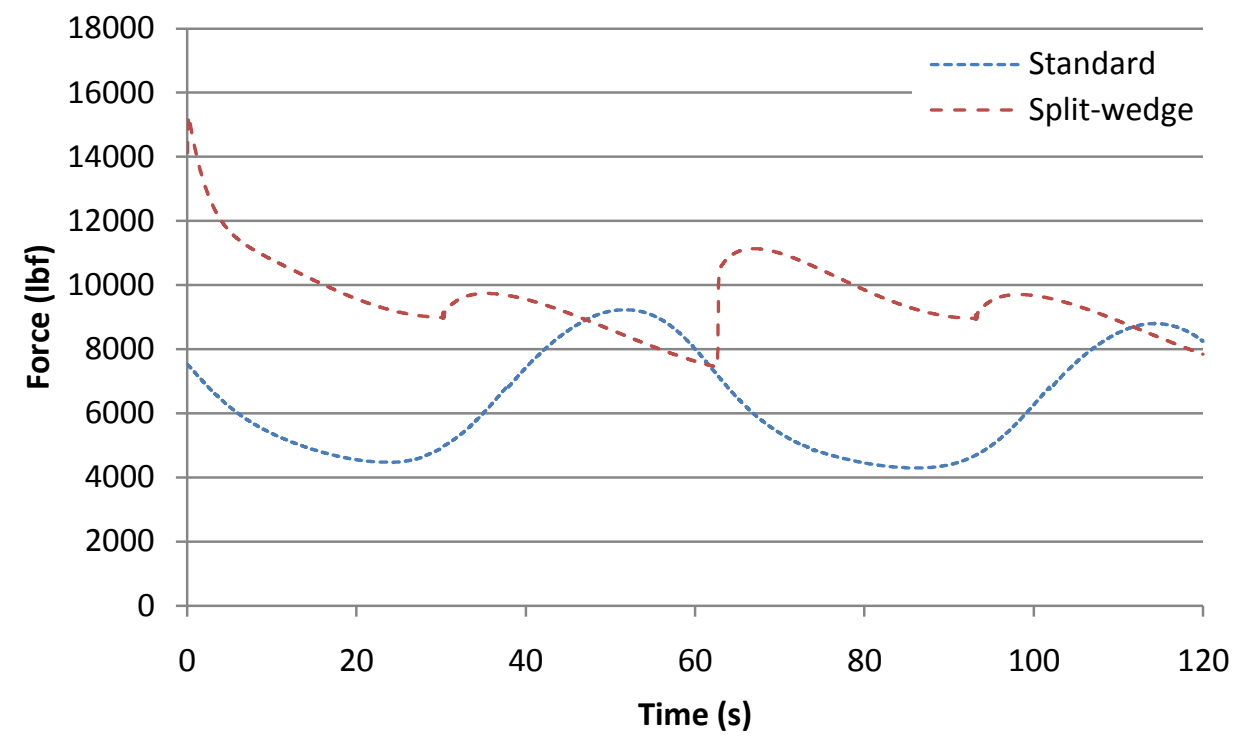

Figure 66. Longitudinal force comparison between standard and split-wedge models

The interactive affects of warp and bounce were also investigated for the split-wedge model. In Figure 67 we can see that the amplitude of displacement is very similar between the pure bounce and warp with bounce case, with the oscillation in the warp with bounce case centered on the displacement of the pure warp case. Figure 68 shows that the addition of the bounce input to the warp input generated an oscillation around the warp stiffness values from the pure warp simulation. This is an expected affect, as each model has shown an overall increase in force when the springs are compressed, and a reduction of force when they are decompressed. In Figure 69 we see that the yaw of the bolster is not affected by the bounce. As was previously mentioned, the wedges are almost constrained to move with the bolster due to the geometry, so even with a bounce input, the wedges are not able to add additional yaw to the bolster. 

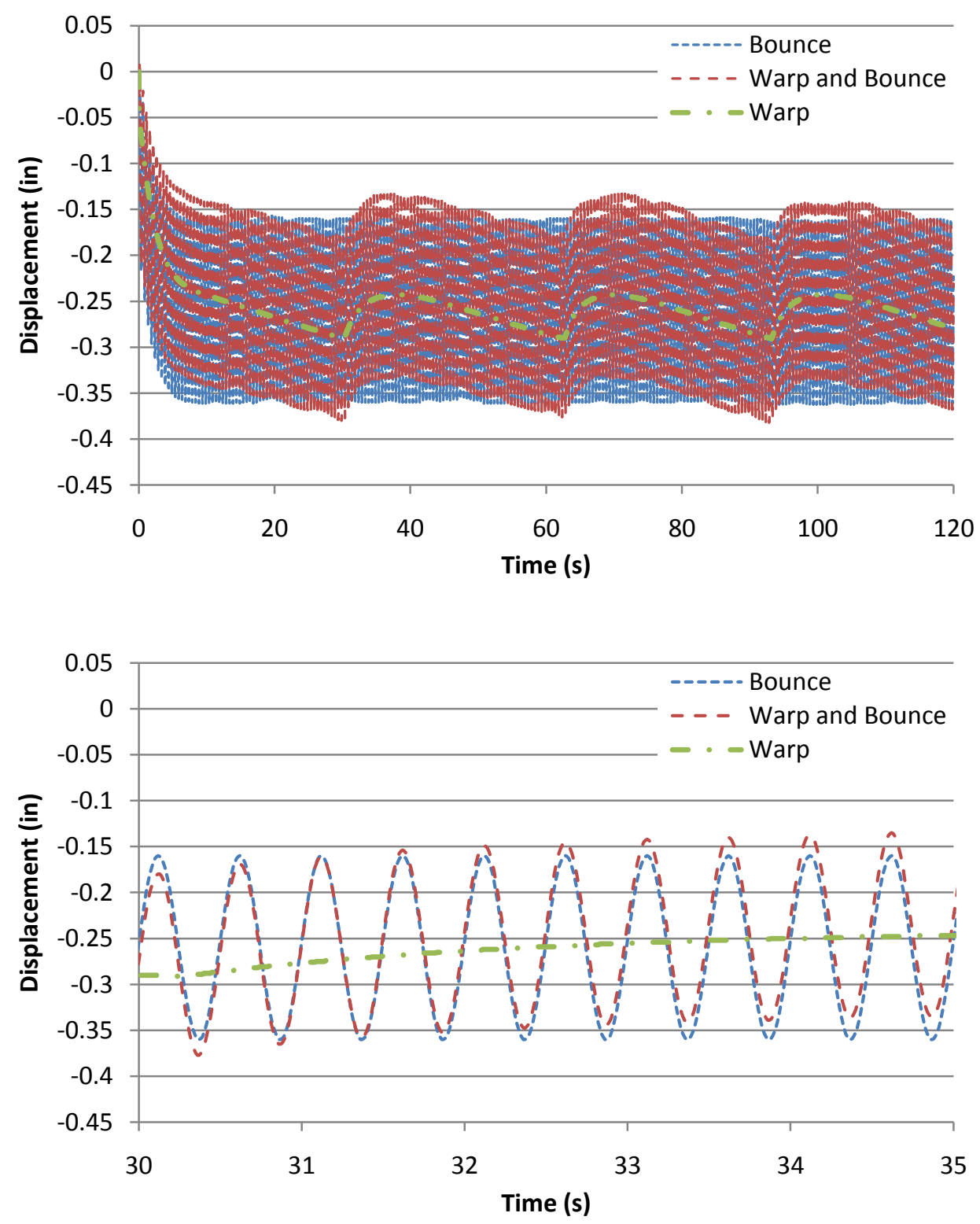

Figure 67. Bolster displacement comparison between pure bounce, pure warp, and warp with bounce 


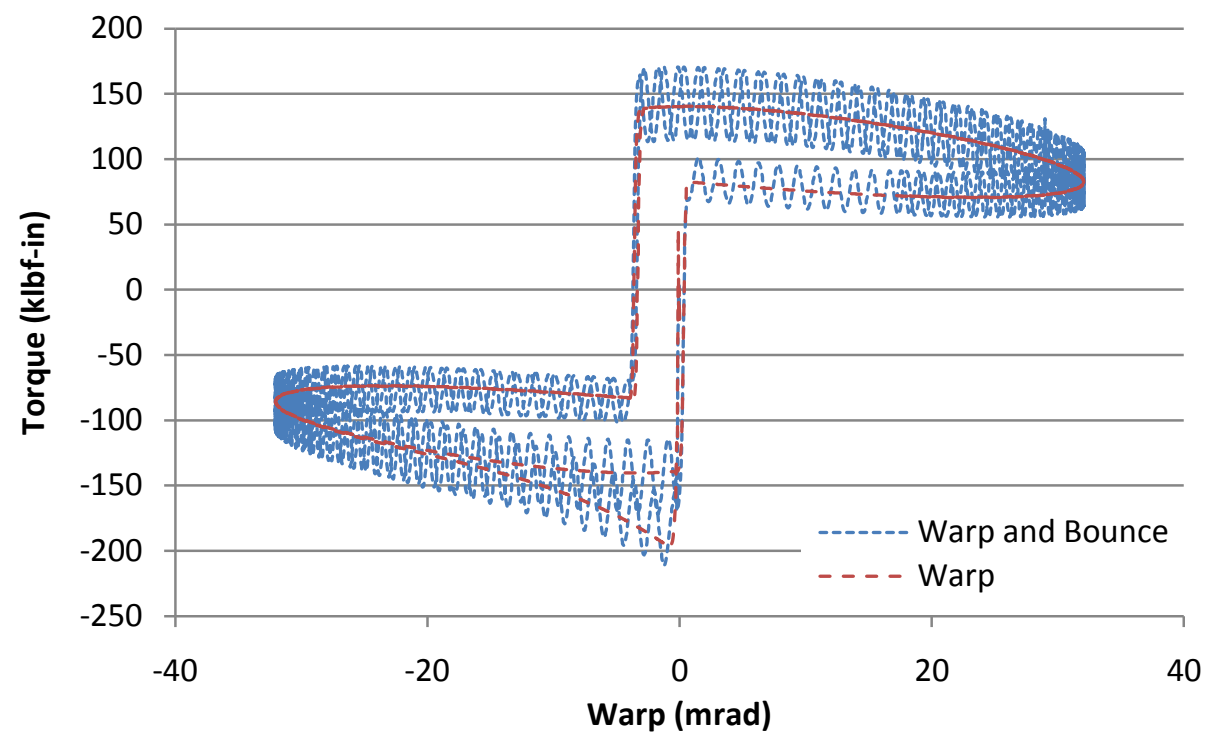

Figure 68. Warp stiffness comparison between pure warp and warp/bounce

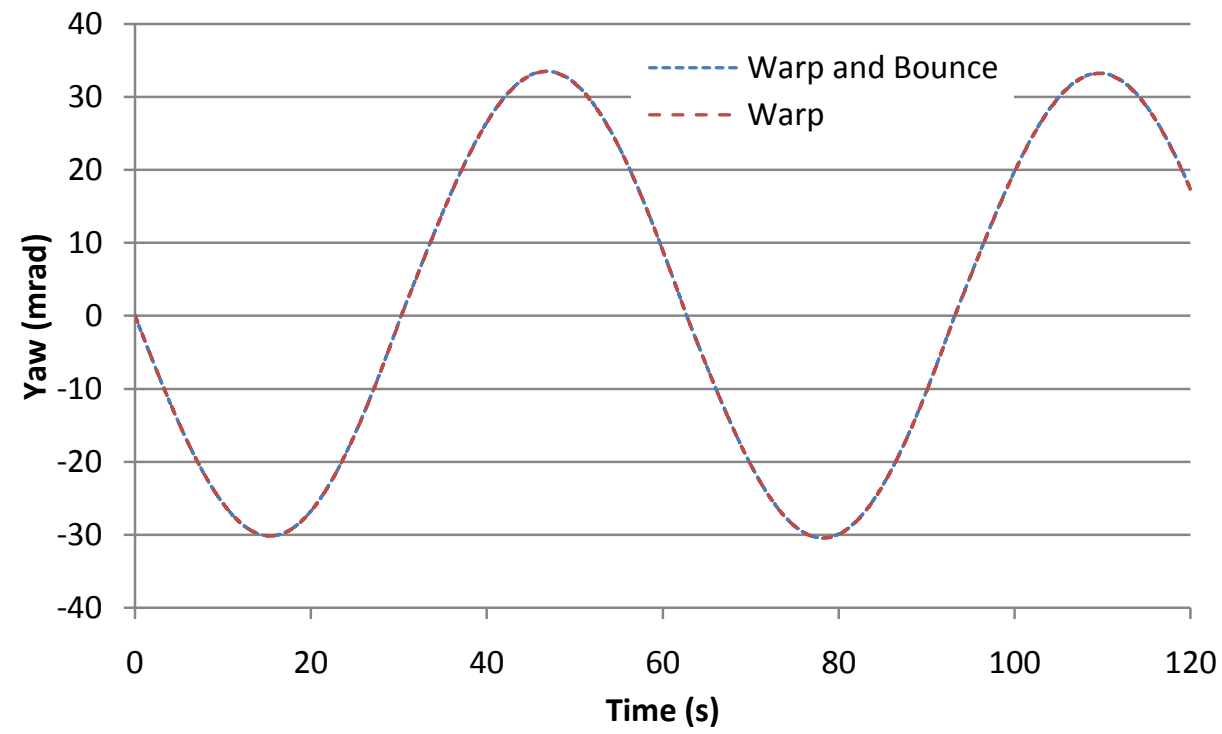

Figure 69. Bolster yaw comparison between pure warp and warp/bounce

When we look at the vertical force on the wedge pair, Figure 70 shows that the oscillation of the force for the warp/bounce input is centered on the vertical force of the pure warp input, as expected. Figure 71 shows that the amplitude of the oscillation is fairly similar for the pure bounce and the warp/bounce inputs. The exception is that with the warp/bounce input, the amplitude of the vertical force oscillation grows right before the force is shifted from one of the wedges to the other in the wedge pair. After this occurs, the amplitude quickly 
returns to the amplitude of the pure bounce input. We also see that there are points during the run where there is a decrease in the total vertical force. This indicates that there is a decrease in the ability of the wedges to damp the system while being warped and bounced, but it appears to be less significant for the split wedge model than for the standard model.
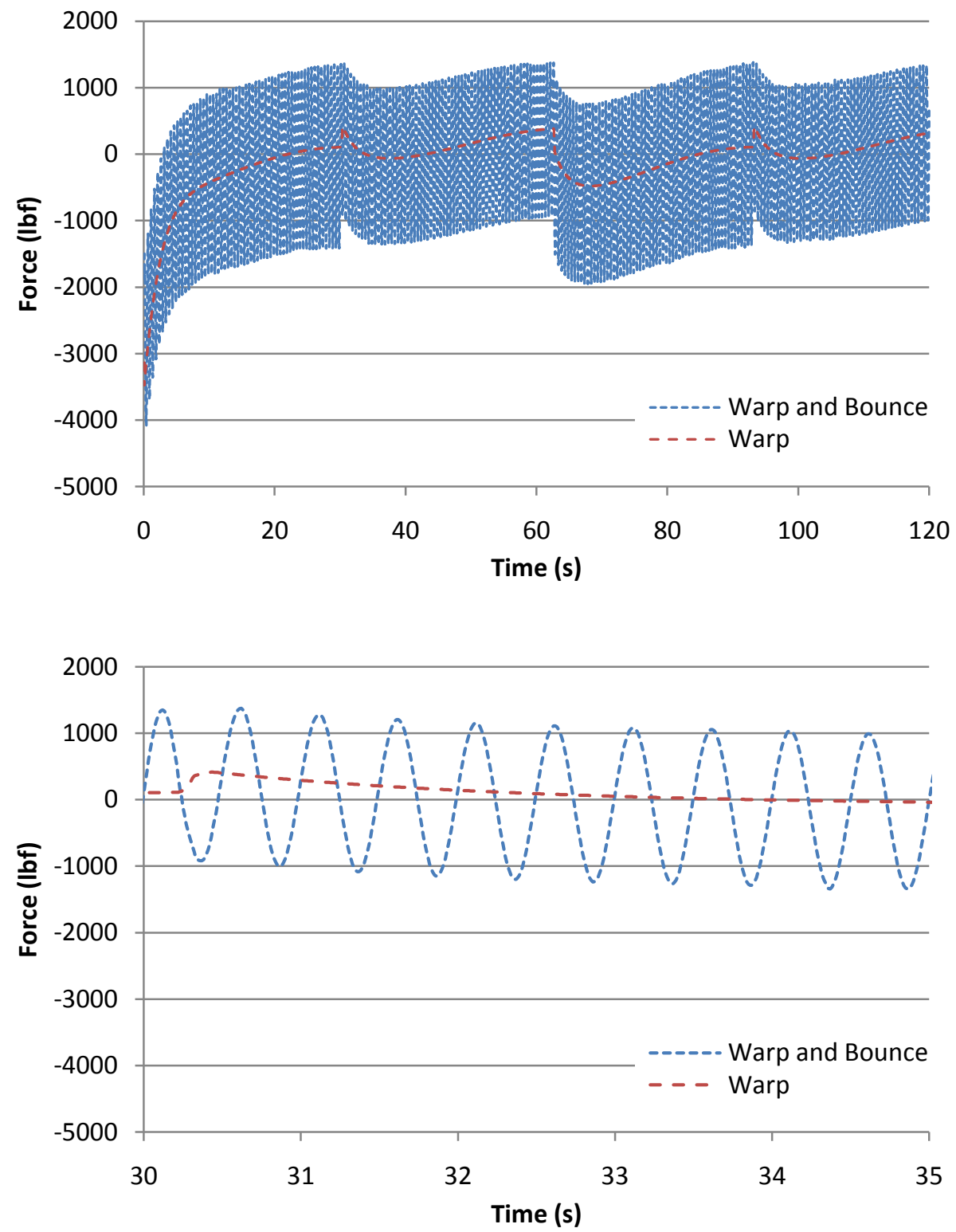

Figure 70. Vertical force comparison between pure warp and warp/bounce 

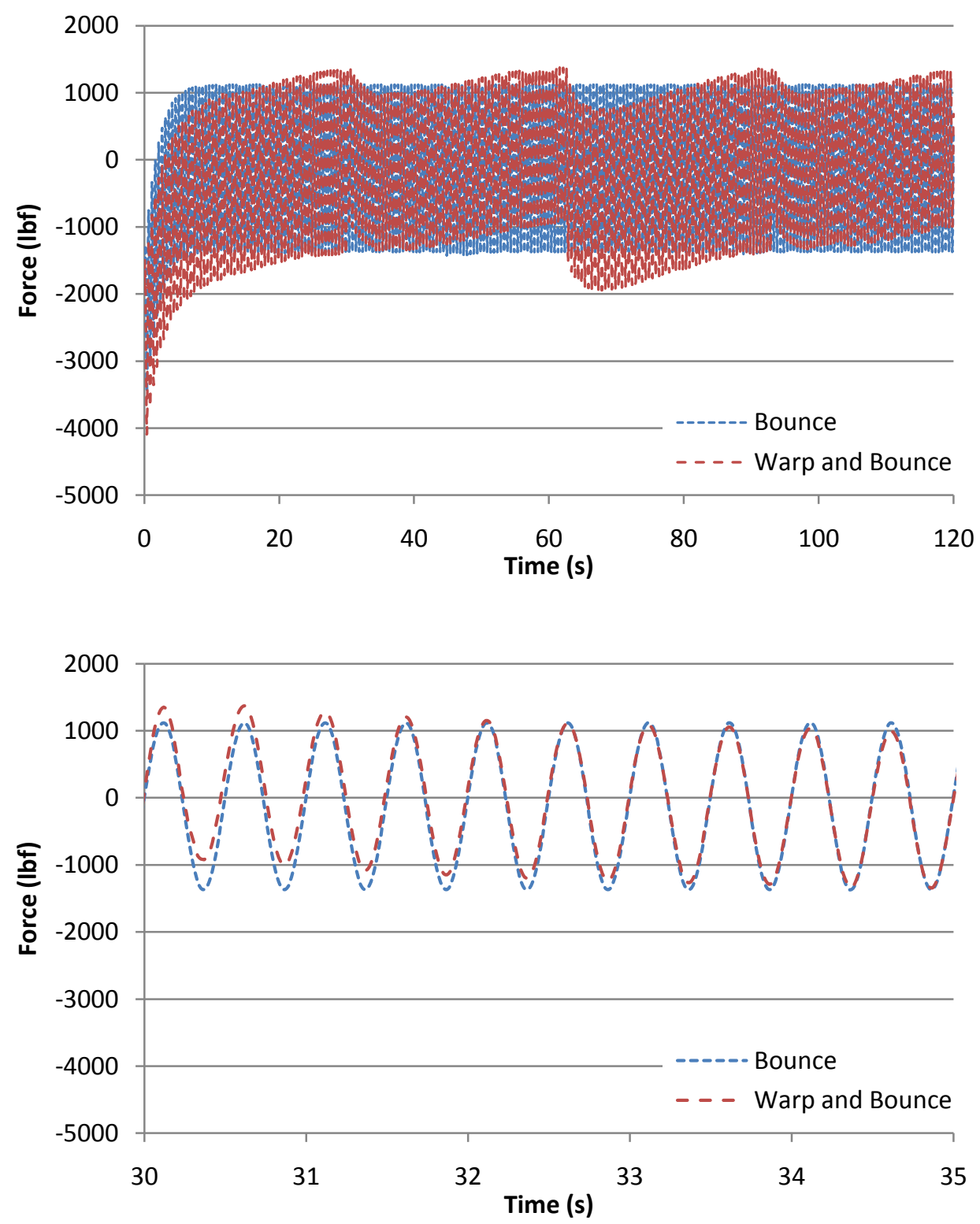

Figure 71. Vertical force comparison between pure bounce and warp/bounce

When determining the affects of the combined warp and bounce on the longitudinal force, Figure 72 shows that the amplitude of the warp/bounce force oscillation is centered on the force values for the pure warp input, as it was for the vertical force. In Figure 73 we see that the warp input increases the longitudinal force oscillation amplitude slightly at the beginning of the motion, and again around 65 seconds, but then the amplitudes become more similar. 

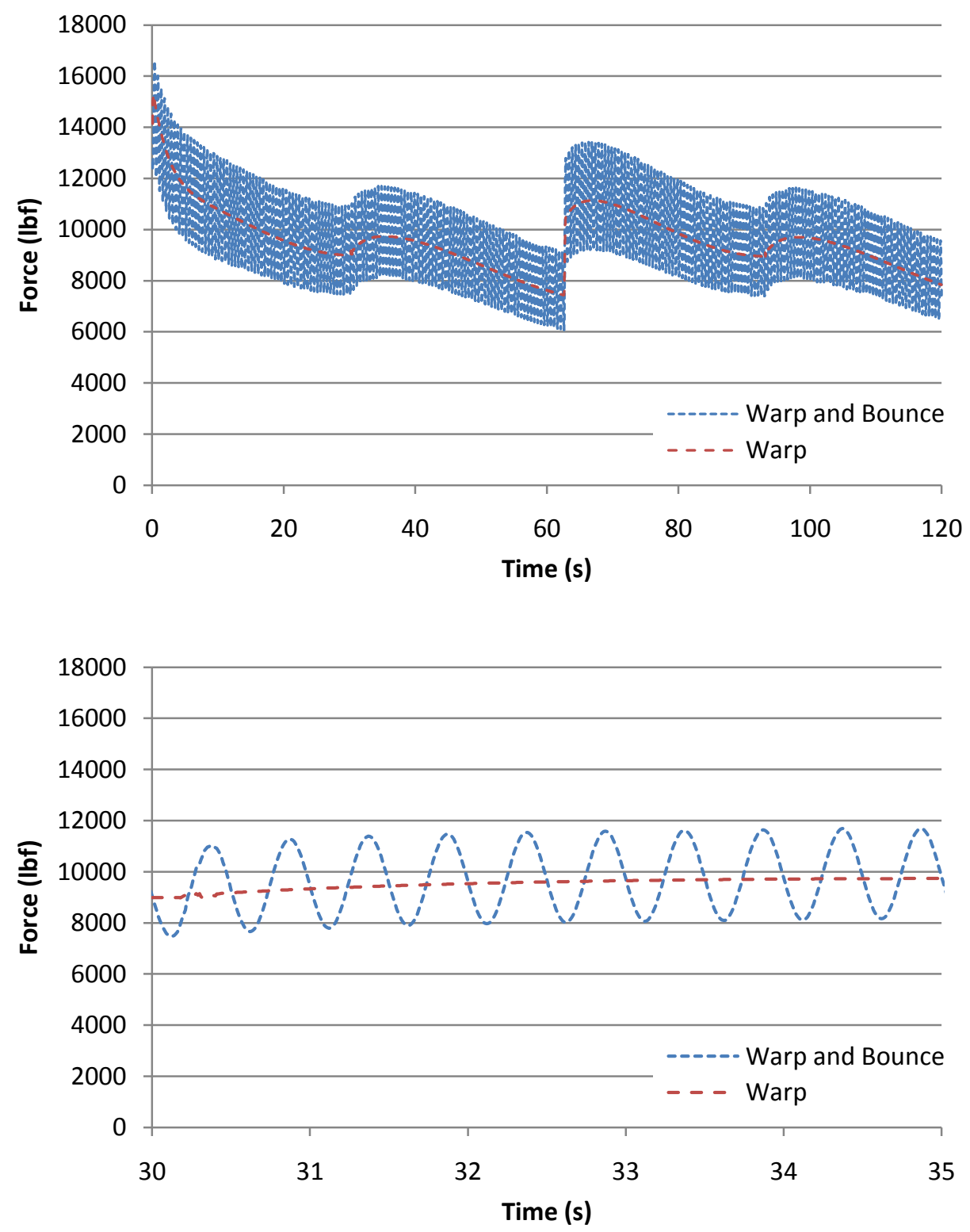

Figure 72. Longitudinal force comparison between pure warp and warp/bounce 

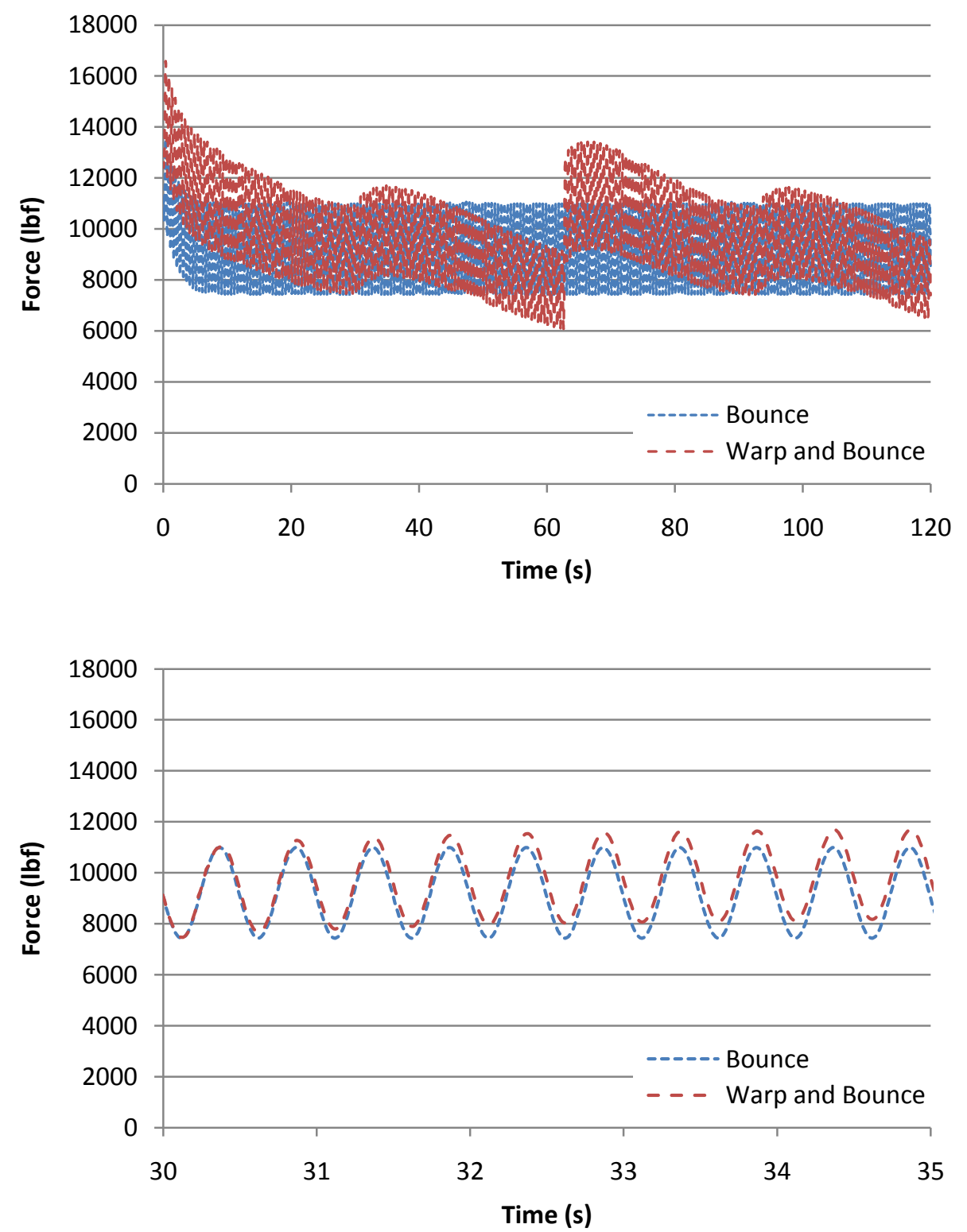

Figure 73. Longitudinal force comparison between pure bounce and warp/bounce 


\section{Chapter 6. Conclusions and Future Work}

This chapter will discuss the conclusions of the thesis, and future work.

\subsection{Conclusions}

The goal of this research was to investigate how the manner of modeling the friction wedge affects the models ability to accurately predict the behavior of the truck system. The most common way of modeling friction wedges in current software programs is to neglect the body geometry and inertial properties, and model the wedge as a connection. While the formulas that model this connection have evolved over the years to be more complex, and thus better capture the behavior, there are still scenarios that cannot be accurately modeled, such as the truck warping case.

The RTL at Virginia Tech has been involved in research into the modeling of friction wedges in the past, and had created quarter-truck and half-truck models in MATLAB ${ }^{\circledR}$ and MAMBO. These models $[2,3,4,5,6]$ were used primarily to investigate bounce inputs with no

warp, or with a constant bolster yaw angle. The research presented in this thesis is a continuation of the prior work done at the RTL.

The main accomplishments of this thesis are summarized below. In this thesis a halftruck model was developed in $\mathrm{LMS}^{\circledR}$ Virtual.Lab, with similar parameters to those of the $M_{A T L A B}{ }^{\circledR}$ models as a means of providing continuity with the prior research at the RTL. Fulltruck models were then developed in $\mathrm{LMS}^{\circledR}$ Virtual.Lab for further research into the dynamic behavior of the truck. The change in software was made to allow for more complex calculations to be performed more quickly and efficiently.

The main focus of the research was to perform warp simulations and to compare them with test data gathered at $\mathrm{TTCl}$ and with simulation results from NUCARS ${ }^{\circledR}$. To accomplish this, a full-truck model was created with similar parameters as the test vehicle used during the test. It was found that the model was able to closely replicate the warp stiffness measured in the test. When compared to $\mathrm{NUCARS}^{\circledR}$, the warp stiffness was considerable larger in the stand- 
alone model developed in this study. Due to the good correlation with the test data, it was concluded that the wedge elements used in NUCARS ${ }^{\circledR}$ are not sufficient in calculating accurate warp stiffness's.

Another research objective was to determine how the interaction between warping and bouncing affects the performance of the wedge. This was done by running three simulations, one with bounce, one with warp, and one with warp and bounce. The bounce amplitude and frequency were the same for the simulations with bounce, and the warp amplitude and frequency was the same for the simulations with warp. The important conclusion from this comparison was that the ability of the wedges to damp the bouncing was diminished by the addition of a warp input. This is can be explained by the fact that there is only a limited amount of friction available in this case. When the system is both warped and bounced, that friction has to be split between the vertical and lateral directions, thus decreasing the ability to damp the vertical motion.

The model was modified to replicate a truck with severely worn sideframes, to compare the behavior of a worn system to an unworn system. In a simple bounce test, we found that the damping ability was greatly diminished, as the amplitude of the bolsters bounce was significantly larger. The warp stiffness was also significantly lower than for the unworn truck. When attempting to determine the interactive effects of warp and bounce, the simulations reached an unsolvable state. The loose fits of the bodies made them more susceptible to getting jammed, which can be a very dangerous situation on the track.

A third model was made, which incorporated split-wedges rather than a standard wedge. We found that in pure bouncing simulations, the split wedge performed very similar to the standard wedge. In the warp simulations, however, the behavior was significantly different. The split-wedges produced an almost constant warp stiffness due to the angled bolster contact surfaces. The interaction between bounce and warp showed a less significant effect for the split wedge than the standard wedge, meaning that the addition of the warp input did not negatively affect the vertical damping as much as with the standard wedge. 


\subsection{Future Work}

Using the current models created in this study, we have been able to gain valuable insight into the behavior of friction wedges, and of the full-truck. We feel that these models are a good starting point, but further improvement of certain aspects of the model will lead to more accurate results in the future. In $\mathrm{LMS}^{\circledR}$ Virtual.Lab, we used a sphere-to-extruded surface contact model for all contact points. This was done to reduce the simulation times significantly, but had the drawback of only allowing for the input of the dynamic coefficient of friction. The CAD contact model allows for both the static and dynamic coefficients of friction, and is a full face body contact model. A comparison between models using these two types of contact would allow us to see what effect the static coefficient of friction would play.

The effect of different loading conditions on the behavior of the truck would be another area that we would like to investigate in the future. We would like to be able to determine how the dynamics of the truck are affected by different loads, as well as sudden changes in loads. Cars are often unloaded very suddenly, so it would be of interest to investigate how the bodies are affected by a sudden change in load.

Another area that would be interesting to examine in the future is the effect of wear on the system. While we were able to run simulations on one specific type of wear, simulations with different wedge and bolster face geometries would allow us to better understand how the truck behavior changes as the wear of the bodies evolve. How the wear occurs is also an area of research that we would like to examine.

The split-wedge model was the last model created for this research. In this model, a limitation was placed on how the wedges of a wedge pair were able to move with respect to each other, as well as with respect to the spring on which they sit. In reality, they are semiindependent bodies, which sit on the same spring. Due to the definition of a spring as a point to point contact, we were only able to give the two halves one degree-of-freedom with respect to each other. In the future we would like to examine a better way of modeling the connection between the wedges and the springs, so that we can allow the two halves more realistic degrees-of-freedom. We would also like to get accurate inertial properties for the split-wedges, 
as the properties used for the simulations run for this research were likely higher than they should be. 


\section{Chapter 7. References}

1. ASF Maintenance \& Repair Maunal, American Steel Foundries, 1998, Chicago, IL.

2. Steets, Jennifer. "A Multibody Dynamics Approach to the Modeling of Friction Wedge Elements for Freight Train Suspensions." Masters thesis, Virginia Tech 2007.

3. Ballew, Brent. "Advanced Multibody Dynamics Modeling of the Freight Train Truck System." Masters thesis, Virginia Tech 2008.

4. Sperry, B., Sandu, C., and Ballew, B., "Complex Bogie Modeling Incorporating Advanced Friction Wedge Components," Proceedings of the 2009 ASME Joint Rail Conference, March 3-5, 2009, Pueblo, CO.

5. Steets, J., Chan, B., Sandu, C., "A Multibody Dynamics Approach to the Modeling of Friction Wedge Elements for Freight Train Suspensions: Part I - Theoretical Modeling." ASCE Journal of Transportation Engineering

6. Steets, J., Chan, B., Sandu, C., Ballew, B., "A Multibody Dynamics Approach to the Modeling of Friction Wedge Elements for Freight Train Suspension: Part II - Simulation and Analysis." ASCE Journal of Transportation Engineering

7. Klauser, P. E. "Modeling Friction Wedges Part I: The State-of-the-Art," Proceedings of IMECE 04, November 13-20, 2004, Anaheim, CA.

8. Klauser, P. E., "Modeling Friction Wedges Part II: An Improved Model," Proceedings of IMECE 04, November 13-20, 2004, Anaheim, CA.

9. Harder, R. F., "Dynamic Modeling and Simulation of Three-Piece North American Freight Vehicle Suspensions with Non-linear Frictional Behavior Using ADAMS/Rail," $5^{\text {th }}$ ADAMS/Rail User's Conference, May 10-12, Haarlem, 2000.

10. Kaiser, A. B., Cusumano, J.P., and Gardner, J. F., "Modeling and Dynamics of Friction Wedge Dampers in Railroad Freight Trucks." International Journal of Vehicle Mechanics and Mobility, 2002.

11. Standard Car Truck Company Manual, Standard Car Truck Company, 2004, Park Ridge, IL. 